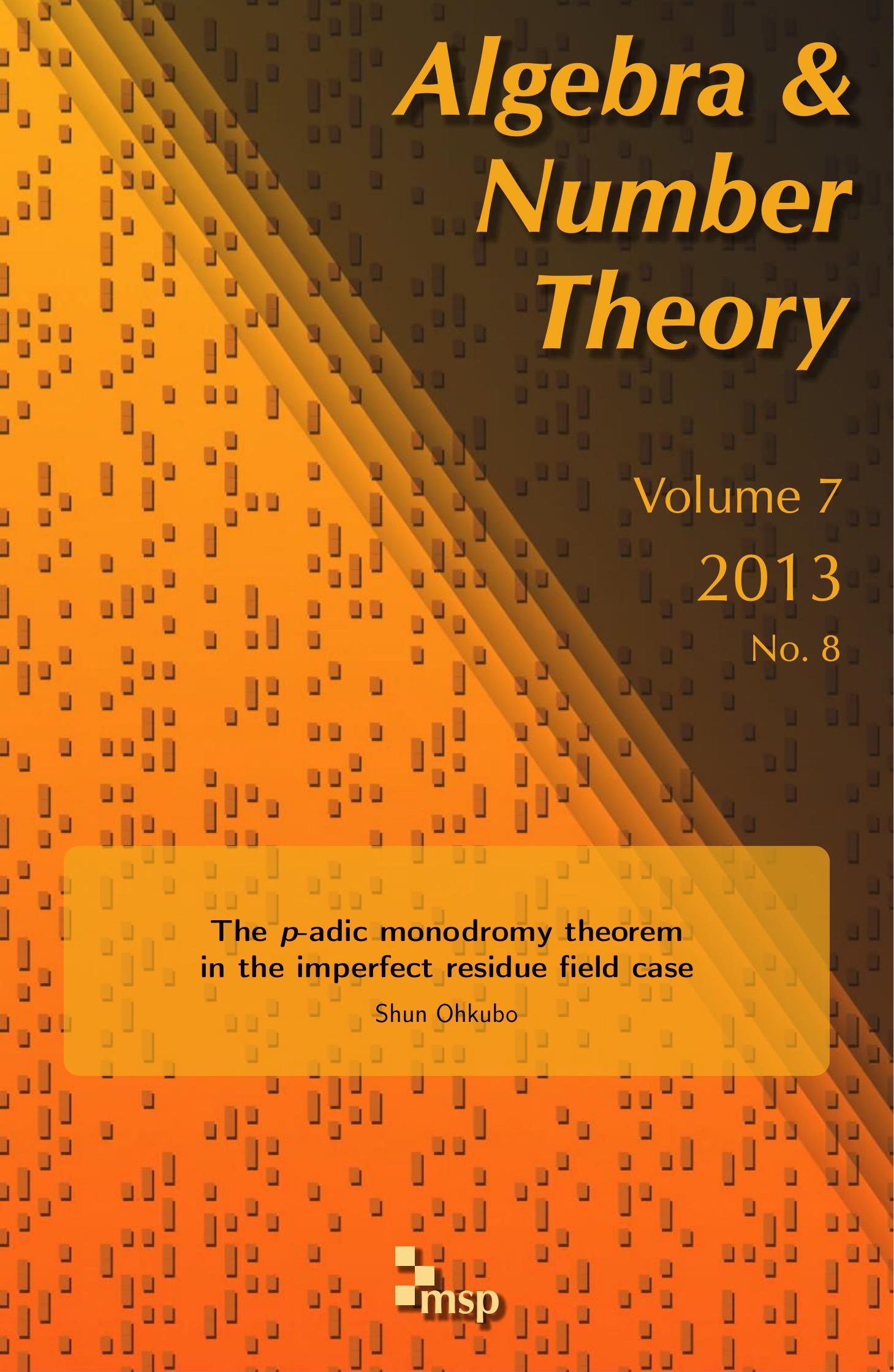




\title{
The $p$-adic monodromy theorem in the imperfect residue field case
}

\author{
Shun Ohkubo
}

Let $K$ be a complete discrete valuation field of mixed characteristic $(0, p)$ and $G_{K}$ the absolute Galois group of $K$. In this paper, we will prove the $p$-adic monodromy theorem for $p$-adic representations of $G_{K}$ without any assumption on the residue field of $K$, for example the finiteness of a $p$-basis of the residue field of $K$. The main point of the proof is a construction of $\left(\varphi, G_{K}\right)$-module $\widetilde{\mathbb{N}}_{\text {rig }}^{\nabla+}(V)$ for a de Rham representation $V$, which is a generalization of Pierre Colmez's $\widetilde{\mathbb{N}}_{\text {rig }}^{+}(V)$. In particular, our proof is essentially different from Kazuma Morita's proof in the case when the residue field admits a finite $p$-basis.

We also give a few applications of the $p$-adic monodromy theorem, which are not mentioned in the literature. First, we prove a horizontal analogue of the $p$-adic monodromy theorem. Secondly, we prove an equivalence of categories between the category of horizontal de Rham representations of $G_{K}$ and the category of de Rham representations of an absolute Galois group of the canonical subfield of $K$. Finally, we compute $H^{1}$ of some $p$-adic representations of $G_{K}$, which is a generalization of Osamu Hyodo's results.

Introduction

1978

Conventions

1981

1. Preliminaries

1983

2. A generalization of Sen's theorem

1993

3. Basic construction of rings of $p$-adic periods

1994

4. Basic properties of rings of $p$-adic periods

2009

5. Construction of $\widetilde{\mathbb{N}}_{\text {rig }}^{\nabla+}(V)$

2014

6. Proof of the Main Theorem

2018

7. Applications

2026

Acknowledgment

2036

References

2036

The author is supported by the Research Fellowships of the Japan Society for the Promotion of Science for Young Scientists.

MSC2010: primary 11F80; secondary 11F85, 11S15, 11S20, 11S25.

Keywords: $p$-adic Hodge theory, $p$-adic representations. 


\section{Introduction}

Let $p$ be a prime and $K$ a complete discrete valuation field of mixed characteristic $(0, p)$ with residue field $k_{K}$. Let $G_{K}$ be the absolute Galois group of $K$. When $k_{K}$ is perfect, Jean-Marc Fontaine defined the notions of crystalline, semistable, de Rham, Hodge-Tate representations for $p$-adic representations of $G_{K}$ (see [Fontaine 1994a; 1994b] for example). The $p$-adic monodromy conjecture, which asserts that de Rham representations are potentially semistable, was first proved by Laurent Berger [2002, Théorème 0.7] by using the theory of $p$-adic differential equations. Precisely speaking, Berger used the $p$-adic local monodromy theorem for $p$-adic differential equations with Frobenius structure due to Yves André, Zoghman Mebkhout, and Kiran Kedlaya.

The notions of the above categories of representations were defined by Olivier Brinon [2006] when $k_{K}$ admits a finite $p$-basis. In this case, the $p$-adic monodromy theorem was proved by Kazuma Morita [2011, Corollary 1.2]. Roughly speaking, he proved the $p$-adic monodromy theorem by studying some differential equations, which are defined by Sen's theory of $\mathbb{B}_{\mathrm{dR}}$ due to Fabrizio Andreatta and Olivier Brinon [2010]. In that reference, Tate-Sen formalism for a quotient $\Gamma_{K}$ of $G_{K}$ is applied to establish Sen's theory of $\mathbb{B}_{\mathrm{dR}}$, where $\Gamma_{K}$ is isomorphic to an open subgroup of $\mathbb{Z}_{p}^{\times} \ltimes \mathbb{Z}_{p}(1)^{J_{K}}$ with $J_{K}:=\operatorname{dim}_{k_{K}} \Omega_{k_{K} / \mathbb{Z}}^{1}<\infty$. To prove Tate-Sen formalism, we iteratively use analogues of the normalized trace map due to John Tate. Hence, we can not use Morita's approach in the case $J_{K}=\infty$.

Our main theorem in this paper is the $p$-adic monodromy theorem without any assumption on the residue field $k_{K}$. We also give the following applications of the $p$-adic monodromy theorem, which are not mentioned in the literature: First, we will prove a horizontal analogue of the $p$-adic monodromy theorem (Theorem 7.4). Secondly, we will prove that the category of horizontal de Rham representations of $G_{K}$ is canonically equivalent to the category of de Rham representations of $G_{K_{\text {can }}}$ (Theorem 7.6), where $K_{\text {can }}$ is the canonical subfield of $K$. Finally, we will calculate $H^{1}$ of horizontal de Rham representations under a certain condition on HodgeTate weights (Theorem 7.8). This calculation is a generalization of calculations done by Hyodo for $\mathbb{Z}_{p}(n)$ with $n \in \mathbb{Z}$ (Theorem 1.16).

Statement of Main Theorem. Let $K$ and $G_{K}$ be as above. We do not put any assumption on the residue field $k_{K}$ of $K$, in particular, we may consider the case that $k_{K}$ is imperfect with $\left[k_{K}: k_{K}^{p}\right]=\infty$. In this setup, the notions of crystalline, semistable, de Rham, Hodge-Tate representations are also defined (see Section 3). Then, our main theorem is the following:

Main Theorem ( $p$-adic monodromy theorem). Let $V$ be a de Rham representation of $G_{K}$. Then, there exists a finite extension $L / K$ such that the restriction $\left.V\right|_{L}$ is semistable. 
Note that the converse can be easily proved by using Hilbert 90 .

Strategy of proof. As is mentioned above, Kazuma Morita's proof can not be generalized directly. When the residue field $k_{K}$ is perfect, an alternative proof of the $p$-adic monodromy theorem due to Pierre Colmez is available, which does not need the theory of $p$-adic differential equations. We will prove the Main Theorem by generalizing Colmez's method. In the following, we will explain our strategy after recalling Colmez's proof in the case that $V$ is a 2-dimensional de Rham representation. (We can prove the higher-dimensional case in a similar way.) After replacing $K$ by the maximal unramified extension of $K$ and taking a Tate twist of $V$, we may also assume that we have $\mathbb{D}_{\mathrm{dR}}(V)=\left(\mathbb{B}_{\mathrm{dR}}^{+} \otimes_{\mathbb{Q}_{p}} V\right)^{G_{K}}$ and $k_{K}$ is separably closed.

In this paragraph, assume that the residue field of $K$ is perfect, that is, $k_{K}$ is algebraically closed. We first fix notation: Let $\tilde{\mathbb{B}}_{\text {rig }}^{+}:=\bigcap_{n \in \mathbb{N}} \varphi^{n}\left(\mathbb{B}_{\text {cris }}^{+}\right)$. For $h \in \mathbb{N}_{>0}$ and $a \in \mathbb{N}$, denote $\mathbb{U}_{h, a}:=\left(\mathbb{B}_{\text {cris }}^{+}\right)^{\varphi^{h}=p^{a}}$ and $\mathbb{U}_{h, a}^{\prime}:=\left(\mathbb{B}_{\mathrm{st}}^{+}\right)^{\varphi^{h}=p^{a}}$. Note that we have $\mathbb{U}_{h, 0}=\mathbb{U}_{h, 0}^{\prime}=\mathbb{Q}_{p^{h}}$, where $\mathbb{Q}_{p^{h}}$ denotes the unramified extension of $\mathbb{Q} p$ with $\left[\mathbb{Q}_{p^{h}}: \mathbb{Q}_{p}\right]=h$. We will recall Colmez's proof: His proof has the following two key ingredients. One is Dieudonné-Manin classification theorem over $\tilde{\mathbb{B}}_{\text {rig. }}^{+}$. Then, he applies this theorem to construct a rank 2 free $\tilde{\mathbb{B}}_{\text {rig }}^{+}$-submodule $\tilde{\mathbb{N}}_{\text {rig }}^{+}(V)$ of $\tilde{\mathbb{B}}_{\text {rig }}^{+} \otimes_{\mathbb{Q}_{p}} V$ with basis $e_{1}, e_{2}$. Moreover, $\tilde{\mathbb{N}}_{\text {rig }}^{+}(V)$ is stable by $\varphi$ and $G_{K}$-actions and the following properties are satisfied:

(i) We have an isomorphism of $\mathbb{B}_{\mathrm{dR}}^{+}\left[G_{K}\right]$-modules

$$
\mathbb{B}_{\mathrm{dR}}^{+} \otimes_{\tilde{\mathbb{B}}_{\mathrm{rg}}^{+}} \tilde{\mathbb{N}}_{\text {rig }}^{+}(V) \cong\left(\mathbb{B}_{\mathrm{dR}}^{+}\right)^{2} .
$$

(ii) There exist $h \in \mathbb{N}_{>0}$ and a 1-cocycle

$$
C: G_{K} \rightarrow\left(\begin{array}{cc}
\mathbb{Q}_{p^{h}}^{\times} & \mathbb{U}_{h, a} \\
0 & \mathbb{Q}_{p^{h}}^{\times}
\end{array}\right) ; \quad g \mapsto C_{g}:=\left(\begin{array}{cc}
\chi_{1}(g) & c_{g} \\
0 & \chi_{2}(g)
\end{array}\right)
$$

such that we have $g\left(e_{1}, e_{2}\right)=\left(e_{1}, e_{2}\right) C_{g}$ for all $g \in G_{K}$.

The second key ingredient is the $H_{g}^{1}=H_{\mathrm{st}}^{1}$-theorem for $\mathbb{U}_{h, a}^{\prime}$ with $h, a \in \mathbb{N}_{>0}$ : Let $L / K$ be a finite extension. If a 1 -cocycle $G_{L} \rightarrow \mathbb{U}_{h, a}^{\prime}$ is a 1-coboundary in $\mathbb{B}_{\mathrm{dR}}^{+}$, then it is a 1-coboundary in $\mathbb{U}_{h, a}^{\prime}$. By using these facts, Colmez proved the Main Theorem as follows. When $h=0$, we may regard $C$ as a $p$-adic representation of $G_{K}$, which is Hodge-Tate of weights 0 by (i). By Sen's theorem on $\mathbb{C}_{p}$-representations, $C$ has a finite image, which implies the assertion. Therefore, we may assume $h>0$. By the cocycle condition of $C, \chi_{i}$ for $i=1,2$ is a character. By (i), $\chi_{i}$ for $i=1,2$ is Hodge-Tate with weights 0 as a $p$-adic representation. By Sen's theorem again, there exists a finite extension $L / K$ such that $\chi_{i}\left(G_{L}\right)=1$ for $i=1,2$. By the cocycle condition of $C$ again, $c: G_{L} \rightarrow \mathbb{U}_{h, a}$ is a 1-cocycle, which is a 1-coboundary in $\mathbb{B}_{\mathrm{dR}}^{+}$ 
by (i). By the $H_{g}^{1}=H_{\mathrm{st}}^{1}$-theorem, there exists $x \in \mathbb{U}_{h, a}^{\prime}$ such that $c_{g}=(g-1)(x)$ for all $g \in G_{L}$. Therefore,

$$
e_{1},-x e_{1}+e_{2} \in \mathbb{B}_{\mathrm{st}}^{+} \otimes_{\tilde{\mathbb{B}}_{\mathrm{rig}}^{+}} \tilde{\mathbb{N}}_{\text {rig }}^{+}(V) \subset \mathbb{B}_{\mathrm{st}}^{+} \otimes_{\mathbb{Q}_{p}} V
$$

form a basis of $\mathbb{D}_{\mathrm{st}}\left(\left.V\right|_{L}\right)$, which implies that $\left.V\right|_{L}$ is semistable.

We will outline our proof of the Main Theorem in the following: For simplicity, we omit some details. We first fix notation: In the imperfect residue field case, we can construct rings of $p$-adic periods $\mathbb{B}_{\text {cris }}^{+}, \mathbb{B}_{\mathrm{st}}^{+}$and $\mathbb{B}_{\mathrm{dR}}^{+}$, on which connections $\nabla$ act. Let $\mathbb{B}_{\text {cris }}^{\nabla+}$ and $\mathbb{B}_{\mathrm{st}}^{\nabla+}$ be the rings of the horizontal sections of $\mathbb{B}_{\text {cris }}^{+}$and $\mathbb{B}_{\mathrm{st}}^{+}$ respectively. Let $\tilde{\mathbb{B}}_{\text {rig }}^{\nabla+}:=\bigcap_{n \in \mathbb{N}} \varphi^{n}\left(\mathbb{B}_{\text {cris }}^{\nabla+}\right)$. For $h \in \mathbb{N}_{>0}$ and $a \in \mathbb{N}$, let $\mathbb{U}_{h, a}:=$ $\left(\mathbb{B}_{\text {cris }}^{\nabla+}\right)^{\varphi^{h}=p^{a}}$ and $\mathbb{U}_{h, a}^{\prime}:=\left(\mathbb{B}_{\text {st }}^{\nabla+}\right)^{\varphi^{h}=p^{a}}$. Even when $k_{K}$ may not be perfect, we can easily prove a generalization of Sen's theorem (Theorem 2.1) and an analogue of Colmez's Dieudonné-Manin classification theorem in an appropriate setting (see Section 5). By using Dieudonné-Manin theorem, we can also give a functorial construction $\tilde{\mathbb{N}}_{\text {rig }}^{\nabla+}(V)$ for a de Rham representation $V$. Our object $\widetilde{\mathbb{N}}_{\text {rig }}^{\nabla+}(V)$ is a rank 2 free $\tilde{\mathbb{B}}_{\text {rig }}^{\nabla+}$-submodule of $\tilde{\mathbb{B}}_{\text {rig }}^{\nabla+} \otimes_{\mathbb{Q}_{p}} V$ with basis $e_{1}, e_{2}$. Moreover, $\widetilde{\mathbb{N}}_{\text {rig }}^{\nabla+}(V)$ is stable by $\varphi$ and $G_{K}$-actions and the following properties are satisfied:

(i) We have an isomorphism of $\mathbb{B}_{\mathrm{dR}}^{+}\left[G_{K}\right]$-modules

$$
\mathbb{B}_{\mathrm{dR}}^{+} \otimes_{\tilde{\mathbb{B}}_{\mathrm{rig}} \nabla} \tilde{\mathbb{N}}_{\text {rig }}^{\nabla+}(V) \cong\left(\mathbb{B}_{\mathrm{dR}}^{+}\right)^{2} .
$$

(ii) There exist $h \in \mathbb{N}_{>0}$ and a 1-cocycle

$$
C: G_{K} \rightarrow\left(\begin{array}{cc}
\mathbb{Q}_{p^{h}}^{\times} & \mathbb{U}_{h, a} \\
0 & \mathbb{Q}_{p^{h}}^{\times}
\end{array}\right) ; \quad g \mapsto C_{g}:=\left(\begin{array}{cc}
\chi_{1}(g) & c_{g} \\
0 & \chi_{2}(g)
\end{array}\right)
$$

such that we have $g\left(e_{1}, e_{2}\right)=\left(e_{1}, e_{2}\right) C_{g}$ for all $g \in G_{K}$.

By using $\tilde{\mathbb{N}}_{\text {rig }}^{\nabla+}(V)$, we prove the Main Theorem as follows. In the case $h=0$, the same proof as above is valid, hence we assume $h>0$. By the cocycle condition of $C, \chi_{i}$ for $i=1,2$ is a character, which is Hodge-Tate with weights 0 by (i). By a generalization of Sen's theorem, we may assume that $\chi_{i}\left(G_{K}\right)=1$ for $i=1,2$ after replacing $K$ by some finite extension. Then, by the cocycle condition of $C$, $c: G_{K} \rightarrow \mathbb{U}_{h, a}$ is a 1-cocycle, which is a 1-coboundary in $\mathbb{B}_{\mathrm{dR}}^{+}$. Unfortunately, an analogue of the above $H_{\mathrm{st}}^{1}=H_{g}^{1}$-theorem does not hold in the imperfect residue field case. Instead, we will prove that there exists $x \in\left(\mathbb{B}_{\text {cris }}^{+}\right)^{G_{K}{ }^{\mathrm{pf}}}$ and $y \in \mathbb{B}_{\mathrm{dR}}^{\nabla+}$ such that $c_{g}=(g-1)(x+y)$ for $g \in G_{K}$ (a special case of Lemma 6.6). Here $K^{\text {pf }}$ denotes a "perfection" of $K$, which is a complete discrete valuation field of mixed characteristic $(0, p)$ with residue field $k_{K}^{\mathrm{pf}}$ and we can regard an absolute Galois group $G_{K^{\mathrm{pf}}}$ of $K^{\mathrm{pf}}$ as a closed subgroup of $G_{K}$. Since we have a canonical isomorphism $\left.\tilde{\mathbb{N}}_{\text {rig }}^{\nabla+}(V)\right|_{G_{K^{\mathrm{pf}}}} \cong \tilde{\mathbb{N}}_{\text {rig }}^{+}\left(\left.V\right|_{G_{K^{\mathrm{pf}}}}\right)$ by functoriality, we can apply Colmez's $H_{g}^{1}=H_{\mathrm{st}^{-}}^{1}$ theorem to the 1-cocycle $\left.c\right|_{G_{K^{\mathrm{pf}}}}$. As a consequence, there exists $z \in \mathbb{U}_{h, a}^{\prime}$ such that 
$c_{g}=(g-1)(z)$ for all $g \in G_{K^{\mathrm{pf}}}$. Since we have $c_{g}=(g-1)(y)$ for all $g \in G_{K^{\mathrm{pf}}}$, we have $z-y \in\left(\mathbb{B}_{\mathrm{dR}}^{\nabla+}\right)^{G_{K \mathrm{pf}}}$, which is included in $\mathbb{B}_{\text {cris }}^{\nabla+}$ by a calculation. Hence, $e_{1},-\{x+(y-z)+z\} e_{1}+e_{2} \in \mathbb{B}_{\mathrm{st}}^{+} \otimes_{\tilde{\mathrm{B}}_{\mathrm{rig}}^{+}} \tilde{\mathbb{N}}_{\text {rig }}^{\nabla+}(V) \subset \mathbb{B}_{\mathrm{st}}^{+} \otimes V$ forms a basis of $\mathbb{D}_{\mathrm{st}}\left(\left.V\right|_{K}\right)$, which implies that $\left.V\right|_{K}$ is semistable.

Structure of paper. In Section 1, we will recall the preliminary facts used in the paper. In Section 2, we will generalize Sen's theorem on $\mathbb{C}_{p}$-admissible representations, which is a special case of the Main Theorem and will be used in the following. The next two sections are devoted to review rings of $p$-adic periods in the imperfect residue field case. Although most of the results seem to be well-known, we will give proofs for the convenience of the reader. In Section 3, we will recall basic constructions and algebraic properties of rings of $p$-adic periods used in $p$-adic Hodge theory in the imperfect residue field case. In Section 4, we will recall Galois-theoretic properties of rings of $p$-adic periods constructed in the previous section. In Section 5, we will construct the $\left(\varphi, G_{K}\right)$-modules $\tilde{\mathbb{N}}_{\text {rig }}^{\nabla+}(V)$ for de Rham representations $V$ after Tate twist. In Section 6, we will prove the Main Theorem combining the results proved in the previous sections. In Section 7, we will give applications of the Main Theorem.

\section{Conventions}

Throughout this paper, let $p$ be a prime and $K$ a complete discrete valuation field of mixed characteristic $(0, p)$. Denote the integer ring of $K$ by $0_{K}$ and a uniformizer of $\mathcal{O}_{K}$ by $\pi_{K}$. Put $U_{K}^{(n)}:=1+\pi_{K}^{n} \mathcal{O}_{K}$ for $n \in \mathbb{N}_{>0}$. Denote by $k_{K}$ the residue field of $K$. We denote by $K^{\text {ur }}$ the $p$-adic completion of the maximal unramified extension of $K$. Denote by $e_{K}$ the absolute ramification index of $K$. For an extension $L / K$ of complete discrete valuation fields, we define the relative ramification index $e_{L / K}$ of $L / K$ by $e_{L / K}:=e_{L} / e_{K}$.

For a field $F$, fix an algebraic closure (resp. a separable closure) of $F$, denote it by $F^{\text {alg }}$ or $\bar{F}$ (resp. $F^{\text {sep}}$ ) and let $G_{F}$ be the absolute Galois group of $F$. For a field $k$ of characteristic $p$, let $k^{\mathrm{pf}}:=k^{p^{-\infty}}$ be the perfect closure in a fixed algebraic closure of $k$. Let $k^{p^{\infty}}:=\bigcap_{n \in \mathbb{N}} k^{p^{n}}$ be the maximal perfect subfield of $k$. Denote by $\mathbb{C}_{p}$ and $\mathbb{O}_{\mathbb{C}_{p}}$ the $p$-adic completion of $\bar{K}$ and its integer ring. Let $v_{p}$ be the $p$-adic valuation of $\mathbb{C}_{p}$ normalized by $v_{p}(p)=1$.

We fix a system of $p$-power roots of unity $\left\{\zeta_{p^{n}}\right\}_{n \in \mathbb{N}_{>0}}$ in $\bar{K}$, that is, $\zeta_{p}$ is a primitive $p$-th root of unity and $\zeta_{p^{n+1}}^{p}=\zeta_{p^{n}}$ for all $n \in \mathbb{N}_{>0}$. Let $\chi: G_{K} \rightarrow \mathbb{Z}_{p}^{\times}$be the cyclotomic character defined by $g\left(\zeta_{p^{n}}\right)=\zeta_{p^{n}}^{\chi(g)}$ for $n \in \mathbb{N}_{>0}$.

For a set $S$, denote by $|S|$ the cardinality of $S$. Let $J_{K}$ be an index set such that we have an isomorphism $\Omega_{k_{K} / \mathbb{Z}}^{1} \cong k_{K}^{\oplus J_{K}}$ as $k_{K}$-vector spaces. In this paper, we do not assume $\left|J_{K}\right|<\infty$. Unless a particular mention is stated, we always fix a 
lift $\left\{t_{j}\right\}_{j \in J_{K}}$ of a $p$-basis of $k_{K}$ and sequences of $p$-power roots $\left\{t_{j}^{p^{-n}}\right\}_{n \in \mathbb{N}, j \in J_{K}}$ in $\bar{K}$, that is, we have $\left(t_{j}^{p^{-n-1}}\right)^{p}=t_{j}^{p^{-n}}$ for $n \in \mathbb{N}_{>0}$.

For a ring $R$, denote the Witt ring with coefficients in $R$ by $W(R)$. If $R$ has characteristic $p$, then we denote the absolute Frobenius on $R$ by $\varphi: R \rightarrow R$ and also denote the ring homomorphism $W(\varphi): W(R) \rightarrow W(R)$ by $\varphi$. Denote by $[x] \in W(R)$ the Teichmüller lift of $x \in R$.

For a $p$-adically Hausdorff abelian group $M$ in which $p$ is not a nonzero divisor, we define the $p$-adic semivaluation of $M$ as the map $v: M \rightarrow \mathbb{Z} \cup\{\infty\}$ such that $v(0)=\infty$ and $v(x)=n$ if $x \in p^{n} M \backslash p^{n+1} M$. We have the properties

$$
v(p x)=1+v(x), \quad v(x+y) \geq \inf (v(x), v(y)), \quad v(x)=\infty \Longleftrightarrow x=0,
$$

for $x, y \in M$. We can extend $v$ to $v: M\left[p^{-1}\right] \rightarrow \mathbb{Z} \cup\{\infty\}$, which we call the $p$-adic semivaluation defined by the lattice $M$. We also call the topology induced by $v$ the $p$-adic topology defined by the lattice $M$.

Let $F$ be a nontrivial nonarchimedean complete valuation field with valuation $v_{F}$. Assume that an $F$-vector space $V$ is endowed with a countable decreasing sequence of valuations $\left\{v^{(n)}: V \rightarrow \mathbb{R} \cup\{\infty\}\right\}_{n \in \mathbb{N}}$ over $F$, that is, we have

$$
\begin{gathered}
v^{(0)}(x) \geq v^{(1)}(x) \geq \cdots, \quad v^{(n)}(\lambda x)=v_{F}(\lambda)+v^{(n)}(x), \\
v^{(n)}(x+y) \geq \inf \left(v^{(n)}(x), v^{(n)}(y)\right)
\end{gathered}
$$

for $\lambda \in F$ and $x, y \in V$. We regard $V$ as a topological $F$-vector space whose topology is generated by $V_{r}^{(n)}:=\left\{x \in V \mid v^{(n)}(x) \geq r\right\}$ for $n, r \in \mathbb{N}$. Then, we call $V$ a Fréchet space (over $F$ ) if $V$ is complete with respect to this topology (see [Schneider 2002, Section 8]). For Fréchet spaces $V$ and $W$, we define the completed tensor product $V \hat{\otimes}_{F} W$ as the inverse limit $\lim _{\longleftarrow, r \in \mathbb{N}} V / V_{r}^{(n)} \otimes_{F} W / W_{r}^{(n)}$ (see [Schneider 2002, Section 17]).

For a multiset $\left\{a_{i}\right\}_{i \in I}$ of elements in $\mathbb{R} \cup\{\infty\}$, we denote $\left\{a_{i}\right\}_{i \in I} \rightarrow \infty$ if the set $\left\{i \in I, a_{i}<n\right\}$ is finite for all $n \in \mathbb{N}_{>0}$. Note that if $|I|<\infty$, then the condition $\left\{a_{i}\right\}_{i \in I} \rightarrow \infty$ is always satisfied.

In this paper, we refer to the continuous cohomology group as the group cohomology. For a profinite group $G$ and a topological $G$-module $M$, denote by $H^{n}(G, M)$ the $n$-th continuous group cohomology with coefficients in $M$. We also denote $H^{0}(G, M)$ by $M^{G}$. We also consider $H^{q}(G, M)$ for $q=0,1$ if $M$ is a (noncommutative) topological $G$-group $M$.

We denote by $\boldsymbol{e}_{i} \in \mathbb{N} \oplus I$ the element whose $i$-th component is equal to 1 and zero otherwise. We will use the following multi-index notation: Let $M$ be a monoid. For a subset $\left\{x_{i}\right\}_{i \in I}$ of $M$ and $\boldsymbol{n}=\left(n_{i}\right)_{i \in I} \in \mathbb{N} \oplus I$, we define $\boldsymbol{x}^{\boldsymbol{n}}:=\prod_{i \in I} x_{i}^{n_{i}}$ and $\boldsymbol{x}^{[\boldsymbol{n}]}:=\prod_{i \in I} u_{i}^{n_{i}} / n_{i}$ ! when it has a meaning. We denote by $|\boldsymbol{n}|$ the sum $\sum_{i \in I} n_{i}$ for 
$\left(n_{i}\right)_{i \in I} \in \mathbb{N} \oplus I$. If no particular mention is stated, for an index set $I$, we denote by $\mathbf{u}_{I}$ or $\mathbf{v}_{I}$ the formal variables $\left\{\mathrm{u}_{i}\right\}_{i \in I}$ or $\left\{\mathrm{v}_{i}\right\}_{i \in I}$ respectively.

For group homomorphisms $f, g: M \rightarrow N$ of abelian groups, we denote by $M^{f=g}$ the kernel of the map $f-g: M \rightarrow N$.

\section{Preliminaries}

This preliminary section is a miscellany of basic definitions, facts, conventions, and remarks used in the paper. Although we will give some proofs for convenience, the reader may skip the proofs by admitting the facts.

1A. Cohen ring. Let $k$ be a field of characteristic $p$. Let $C(k)$ be a Cohen ring of $k$, that is, a complete discrete valuation ring with maximal ideal generated by $p$ and residue field $k$. This is unique up to a canonical isomorphism if $k$ is perfect (in fact, $C(k) \cong W(k)$ ) and unique up to noncanonical isomorphisms in general. Denote $J_{C(k)\left[p^{-1}\right]}$ by $J$ for a while. For a lift $\left\{t_{j}\right\}_{j \in J} \subset C(k)$ of a $p$ basis of $k$, we regard $C(k)$ as a $\mathbb{Z}\left[T_{j}\right]_{j \in J}$-algebra by $T_{j} \mapsto t_{j}$. This morphism is formally étale for the $p$-adic topologies. In fact, we may replace $\mathbb{Z}\left[T_{j}\right]_{j \in J}$ by $R:=\left(\mathbb{Z}\left[T_{j}\right]_{j \in J}\right)_{(p)}$. Since $C(k) / R$ is flat and $k / \mathbb{F}_{p}\left(T_{j}\right)_{j \in J}$ is formally étale for the discrete topologies, $C(k) / R$ is formally étale by [Grothendieck 1964, 0.19.7.1 and 0.20.7.5].

By the lifting property, we have $C\left(k_{K}\right) \rightarrow \mathrm{O}_{K}$, an injective algebra homomorphism which is totally ramified of degree $e_{K}$. We will denote by $K_{0}$ the fraction field of the image of $C(k)$ in $K$. We also note that ${ }^{O_{K}}$ is unique if $k_{K}$ is perfect and nonunique otherwise. By the lifting property again, we have a lift $\varphi:{ }^{0} K_{0} \rightarrow \mathbb{O}_{K_{0}}$ of the absolute Frobenius of $k_{K}$ : It is unique if $k_{K}$ is perfect and nonunique otherwise. An example of such a $\varphi$ is $\varphi\left(t_{j}\right)=t_{j}^{p}$ for all $j \in J_{K_{0}}$. Moreover, when $k_{K}$ is imperfect, the construction of $K_{0}$ cannot be functorial in the following sense: For a finite extension $L / K$, we cannot always choose $K_{0} \subset K$ and $L_{0} \subset L$ such that $K_{0} \subset L_{0}$.

Finally, note that for a given lift $\left\{t_{j}\right\}_{j \in J_{K}} \subset \mathcal{O}_{K}$ of a $p$-basis of $k_{K}$, we can choose $\mathbb{O}_{K_{0}}$ such that $\left\{t_{j}\right\}_{j \in J_{K}} \subset \mathcal{O}_{K_{0}}$. In fact, we regard $\mathbb{O}_{K}$ as a $\mathbb{Z}\left[T_{j}\right]_{j \in J_{K}}$ algebra by sending $T_{j}$ to $t_{j}$. We choose a lift $\left\{t_{j}^{\prime}\right\}_{j \in J_{K}} \subset C\left(k_{K}\right)$ of the $p$ basis $\left\{\bar{t}_{j}\right\}_{j \in J_{K}} \subset k_{K}$ and we regard $C\left(k_{K}\right)$ as a $\mathbb{Z}\left[T_{j}\right]_{j \in J_{K}}$-algebra by $T_{j} \mapsto t_{j}^{\prime}$. Then, we lift the projection $C\left(k_{K}\right) \rightarrow k_{K}$ to a $\mathbb{Z}\left[T_{j}\right]_{j \in J_{K}}$-algebra homomorphism $C\left(k_{K}\right) \rightarrow \mathrm{O}_{K}$ by the lifting property, whose image satisfies the condition. Thus, if we choose a lift $\left\{t_{j}\right\}_{j \in J_{K}}$ of a $p$-basis of $k_{K}$, we may always assume that we have $\left\{t_{j}\right\}_{j \in J_{K}} \subset K_{0}$.

1B. Canonical subfield. We first recall the following two lemmas, which are proved in [Epp 1973, 0.4]. We give proofs for the reader. 
Lemma 1.1. Let $k$ be a field of characteristic $p$.

(i) The field $k^{p^{\infty}}$ is algebraically closed in $k$. In particular, the fields $\left(k^{p^{\infty}}\right)^{\mathrm{sep}}$ and $k$ are linearly disjoint over $k^{p^{\infty}}$.

(ii) For a finite extension $k^{\prime} / k^{p^{\infty}}$, we have $k^{\prime}=\left(k k^{\prime}\right)^{p^{\infty}}$.

Proof.

(i) The assertion follows from the fact that any algebraic extension over a perfect field is perfect.

(ii) As is mentioned in the above proof, $k^{\prime}$ is perfect. We have $k k^{\prime}=k \otimes_{k} p^{\infty} k^{\prime}$ by (i). Hence, we have $\left(k k^{\prime}\right)^{p^{n}}=k^{p^{n}} \otimes_{k} p^{\infty} k^{\prime}$ and

$$
\left(k k^{\prime}\right)^{p^{\infty}}=\bigcap_{n}\left(k^{p^{n}} \otimes_{k^{p}} \infty k^{\prime}\right)=k^{p^{\infty}} \otimes_{k^{p}} \infty k^{\prime}=k^{\prime} .
$$

Lemma 1.2. Let $l / k$ be an algebraic extension of fields of characteristic $p$.

(i) If $l / k$ is a (possibly infinite) Galois extension, then $l^{p^{\infty}} / k^{p^{\infty}}$ is also a (possible infinite) Galois extension. Moreover, the canonical map

$$
G_{l / k} \rightarrow G_{l^{p}} / k^{p^{\infty}}
$$

is surjective.

(ii) If $l / k$ is finite, then $l^{p^{\infty}} / k^{p^{\infty}}$ is also a finite extension. Moreover, we have $\left[l^{p^{\infty}}: k^{p^{\infty}}\right] \leq[l: k]$.

Proof. (i) We may easily reduce to the case that $l / k$ is finite Galois. Obviously any $k$-algebra endomorphism on $l$ induces a $k^{p^{n}}$-algebra endomorphism on $l^{p^{n}}$. In particular, $l^{p^{n}}$ and $l^{p^{\infty}}$ are $G_{l / k}$-stable. Since the Frobenius commutes with the action of $G_{l / k}$, we have $\left(l^{p^{n}}\right)^{G_{l / k}}=\left(l^{G_{l / k}}\right)^{p^{n}}=k^{p^{n}}$. By taking the intersection, we have $\left(l^{p^{\infty}}\right)^{G_{l / k}}=k^{p^{\infty}}$. For $x \in l^{p^{\infty}}$, let $f(X) \in k[X]$ be the monic irreducible separable polynomial such that $f(x)=0$. Then all the solutions of $f$ belong to $l^{p^{\infty}}$ and we have $f(X) \in\left(l^{p^{\infty}}\right)^{G_{l / k}}[X]=k^{p^{\infty}}[X]$. This implies that $l^{p^{\infty}} / k^{p^{\infty}}$ is a Galois extension. The latter assertion follows from the equality $\left(l^{p^{\infty}}\right)^{G_{l / k}}=k^{p^{\infty}}$.

(ii) We may assume that $l / k$ is purely inseparable or separable. If $l / k$ is purely inseparable, then $l$ is generated by finitely many elements of the form $x^{p^{-n}}$ with $n \in \mathbb{N}$ and $x \in k$ as a $k$-algebra. Hence we have $l^{p^{n}} \subset k$ for some $n$, that is, $k^{p^{\infty}}=l^{p^{\infty}}$. Assume that $l / k$ is separable. The first assertion is reduced to the case that $l / k$ is a Galois extension, which follows from (i). Since the canonical $k$-algebra homomorphism $l^{p^{\infty}} \otimes_{k^{p}} \infty k \rightarrow l$ is injective by Lemma 1.1(i), we have $\left[l^{p^{\infty}}: k^{p^{\infty}}\right] \leq[l: k]$.

Defintion 1.3. (i) (Compare [Hyodo 1986, Theorem 2].) We define the canonical subfield $K_{\text {can }}$ of $K$ as the algebraic closure of $W\left(k_{K}^{p^{\infty}}\right)\left[p^{-1}\right]$ in $K$. 
(ii) (Compare [Hyodo 1986, (0-5)].) We define condition (H) as follows:

$K$ contains a primitive $p^{2}$-th root of unity and we have $e_{K / K_{\text {can }}}=1$.

Note that $K_{\text {can }}$ is a complete discrete valuation field of mixed characteristic $(0, p)$ with perfect residue field $k_{K}^{p^{\infty}}$. If $k_{K}$ is perfect, then we have $K_{\text {can }}=K$. We also note that the restriction $G_{K} \rightarrow G_{K_{\text {can }}}$ is surjective since $K_{\text {can }}$ is algebraically closed in $K$. We will regard $G_{K_{\text {can }}}$ as a quotient of $G_{K}$ in the rest of the paper.

Remark 1.4. (i) In [Brinon 2006, Notation 2.29], $K_{\text {can }}$ is denoted by $K^{\nabla}$ since $K_{\text {can }}$ coincides with the kernel of the canonical derivation $d: K \rightarrow \widehat{\Omega}_{K}^{1}$ (Proposition 1.13 below).

(ii) The canonical morphism

$$
K_{\text {can }} \otimes_{K_{\text {can }, 0}} K_{0} \rightarrow K
$$

is injective since we have $e_{K_{0} / K_{\text {can }, 0}}=1$ and $K_{\text {can }} / K_{\text {can }, 0}$ is totally ramified. Note that we have $e_{K / K_{\text {can }}}=1$ if and only if the above morphism is surjective.

The following are the basic properties of the canonical subfields used in this paper.

Lemma 1.5. Let $L / K$ be a finite extension.

(i) The fields $\left(K_{\mathrm{can}}\right)^{\mathrm{alg}}$ and $K$ are linearly disjoint over $K_{\mathrm{can}}$.

(ii) If $L / K$ is Galois, then $L_{\mathrm{can}} / K_{\mathrm{can}}$ is also a finite Galois extension. Moreover, the canonical map $G_{L / K} \rightarrow G_{L_{\text {can }} / K_{\text {can }}}$ is surjective.

(iii) The field extension $L_{\mathrm{can}} / K_{\mathrm{can}}$ is finite with $\left[L_{\mathrm{can}}: K_{\mathrm{can}}\right] \leq[L: K]$.

(iv) If $K^{\prime} / K_{\mathrm{can}}$ is a finite extension, then we have $\left(K K^{\prime}\right)_{\mathrm{can}}=K^{\prime}$.

Proof. (i) Since $K_{\text {can }}$ is algebraically closed in $K$, we have $\left(K_{\text {can }}\right)^{\text {alg }} \cap K=K_{\text {can }}$, which implies the assertion.

(ii) Since $k_{L}^{p^{\infty}} / k_{K}^{p^{\infty}}$ is finite by Lemma 1.2(ii), we have $L_{\text {can }}=L \cap\left(K_{\text {can }}\right)^{\text {alg }}$. Hence we have $L_{\text {can }} \cap K=K_{\text {can }}$. Since $L_{\text {can }} / K_{\text {can }}$ is algebraic, $L_{\text {can }}$ and $K$ are linearly disjoint over $K_{\text {can }}$ by (i). Let $x \in L_{\text {can }}$ and $f(X) \in K_{\text {can }}[X]$ be the monic irreducible polynomial such that $f(x)=0$. By the linearly disjointness, $f(X)$ is irreducible in $K[X]$. Since $L / K$ is Galois, all the solutions of $f(X)=0$ belong to $L \cap\left(K_{\text {can }}\right)^{\text {alg }}=L_{\text {can }}$. This implies that $L_{\text {can }} / K_{\text {can }}$ is Galois. Since we have $\left(L_{\text {can }}\right)^{G_{L / K}}=L_{\text {can }} \cap K=K_{\text {can }}$, we have the rest of the assertion.

(iii) The finiteness of $L_{\text {can }} / K_{\text {can }}$ is reduced to the case that $L / K$ is Galois, which follows from (ii). Since the canonical $K$-algebra homomorphism $L_{\text {can }} \otimes_{K_{\text {can }}} K \rightarrow L$ is injective by (i), we have $\left[L_{\text {can }}: K_{\text {can }}\right] \leq[L: K]$.

(iv) The assertion follows from the inequalities

$$
\left[K^{\prime}: K_{\mathrm{can}}\right] \leq\left[\left(K K^{\prime}\right)_{\mathrm{can}}: K_{\mathrm{can}}\right] \leq\left[K K^{\prime}: K\right]=\left[K^{\prime}: K_{\mathrm{can}}\right],
$$


where the second inequality follows from (iii) and the last equality follows from the linear disjointness of $K$ and $K^{\prime}$ over $K_{\text {can }}$ by (i).

Theorem 1.6 (the complete case of Epp's theorem [1973]). There exists a finite Galois extension of $K^{\prime} / K_{\text {can }}$ such that $K K^{\prime}$ satisfies condition $(H)$.

Proof. By the original Epp's theorem, we have a finite extension $K^{\prime} / K_{\text {can }}$ such that we have $e_{K K^{\prime} / K^{\prime}}=1$. We have only to prove that we have $e_{K K^{\prime \prime} / K^{\prime \prime}}=1$ for any finite extension $K^{\prime \prime} / K^{\prime}$. In fact, if we choose $K^{\prime \prime}$ as the Galois closure of $K^{\prime}\left(\mu_{p^{2}}\right)$ over $K_{\text {can }}$, then $K^{\prime \prime}$ satisfies the condition by Lemma 1.5(iv). Since we have $K K^{\prime \prime}=\left(K K^{\prime}\right) \otimes_{K^{\prime}} K^{\prime \prime}$ by Lemma 1.5(i) and (iv), we have $e_{K K^{\prime \prime} / K K^{\prime}} \leq e_{K^{\prime \prime} / K^{\prime}}$. By multiplying with $e_{K K^{\prime}}=e_{K^{\prime}}$, we have $e_{K K^{\prime \prime}} \leq e_{K^{\prime \prime}}$, implying the assertion.

Example 1.7 (the higher-dimensional local fields case). We say that $K$ has a structure of a higher-dimensional local field if $K$ is isomorphic to a finite extension over the fractional field of a Cohen ring of the field

$$
\mathbb{F}_{q}\left(\left(X_{1}\right)\right)\left(\left(X_{2}\right)\right) \ldots\left(\left(X_{d}\right)\right)
$$

with $q=p^{f}$ (see [Zhukov 2000] about higher-dimensional local fields). In this case, $K_{\text {can }}$ coincides with the algebraic closure of $\mathbb{Q}_{p}$ in $K$. In fact, we have only to prove that $k_{K}^{p^{\infty}}$ is a finite field. By Lemma 1.2(ii), we may reduce to the case $k_{K}=\mathbb{F}_{q}\left(\left(X_{1}\right)\right) \ldots\left(\left(X_{d}\right)\right)$. Then, the assertion follows from an iterative use of the following fact: If $k$ is a field of characteristic $p$, then we have $k((X))^{p^{\infty}}=k^{p^{\infty}}$. Obviously, the RHS is contained in the LHS. Let $f=\sum_{n \gg-\infty} a_{n} X^{n} \in k((X))^{p^{\infty}}$ with $a_{n} \in k$. Since $f \in k((X))^{p}$, we have $a_{n}=0$ if $p \nmid n$ and $a_{n} \in k^{p}$ otherwise. By repeating this argument, we have $a_{n}=0$ for $n \neq 0$ and $f=a_{0} \in k^{p^{\infty}}$.

\section{C. Canonical derivation.}

Defintion 1.8 (Compare [Hyodo 1986, Section 4].). Let $q \in \mathbb{N}$. For a complete discrete valuation ring $R$ with mixed characteristic $(0, p)$, let

$$
\widehat{\Omega}_{R}^{q}:=\lim _{\leftarrow n} \Omega_{R / \mathbb{Z}}^{q} / p^{n} \Omega_{R / \mathbb{Z}}^{q}
$$

and let $d: R \rightarrow \widehat{\Omega}_{R}^{1}$ be the canonical derivation. Let $\widehat{\Omega}_{R\left[p^{-1}\right]}^{q}:=\widehat{\Omega}_{R}^{q}\left[p^{-1}\right]$ for $q \in \mathbb{Z}$ and let $d: R\left[p^{-1}\right] \rightarrow \widehat{\Omega}_{R\left[p^{-1}\right]}^{1}$ be the canonical derivation and $d_{q}: \widehat{\Omega}_{R\left[p^{-1}\right]}^{q} \rightarrow$ $\widehat{\Omega}_{R\left[p^{-1}\right]}^{q+1}$ the morphism induced by the exterior derivation, which satisfies the usual formula $d_{q}(\lambda \omega)=\lambda d_{q} \omega+(-1)^{q} \omega \wedge d \lambda$ for $\lambda \in K$ and $\omega \in \widehat{\Omega}_{K}^{q}$. We endow $\widehat{\Omega}_{R\left[p^{-1}\right]}^{q}$ with the $p$-adic topology defined by the lattice $\operatorname{Im}\left(\widehat{\Omega}_{R}^{q} \stackrel{\text { can }}{\longrightarrow}_{\Omega_{R\left[p^{-1}\right]}^{q}}\right)$. Obviously, the derivation $d_{q}$ is continuous.

For $q \in \mathbb{Z}_{<0}$, we put $\hat{\Omega}_{R\left[p^{-1}\right]}^{q}:=0$ as a matter of convention.

The following are the basic properties of the canonical derivations used in the sequel. 
Lemma 1.9. Let $R$ be a discrete valuation ring with uniformizer $\pi_{R}$ and $\alpha$ : $M \rightarrow M^{\prime}$ a morphism of $R$-modules whose kernel and cokernel are killed by $\pi_{R}^{c}$ for $c \in \mathbb{N}$. Then, for any $R$-module $M^{\prime \prime}$, the kernel and cokernel of the morphism id $\otimes \alpha: M^{\prime \prime} \otimes_{R} M \rightarrow M^{\prime \prime} \otimes_{R} M^{\prime}$ are killed by $\pi_{R}^{2 c}$. In particular, the kernel and cokernel of $\alpha^{\otimes q}: M^{\otimes q} \rightarrow M^{\prime \otimes q}$ are killed by $\pi_{R}^{2 q c}$.

Proof. We prove the first assertion. If $\alpha$ is injective or surjective, then the cokernel and kernel are killed by $\pi_{R}^{c}$ by the calculation of $\operatorname{Tor}_{R}^{\bullet}$. The general case follows easily from these cases by writing $\alpha$ as a composition of an injection and a surjection.

The last assertion follows from the following decomposition and induction on $q$ : $M^{\otimes(q+1)}=M \otimes_{R} M^{\otimes q} \stackrel{\mathrm{id} \otimes \alpha \otimes q}{\longrightarrow} M \otimes_{R} M^{\prime \otimes q} \stackrel{\alpha \otimes \mathrm{id}}{\longrightarrow} M^{\prime} \otimes_{R} M^{\prime \otimes q}=M^{\prime \otimes(q+1)}$

Lemma 1.10 [Hyodo 1986]. Let $q \in \mathbb{N}$.

(i) We have the ${ }^{{ }^{K}} K_{0}$-linear isomorphism $\hat{\Omega}_{\mathscr{O}_{K_{0}}}^{q} \cong \lim _{\longleftarrow}\left(\left(\mathcal{O}_{K_{0}} / p^{n} \mathscr{O}_{K_{0}}\right) \otimes_{\mathbb{Z}} \wedge_{\mathbb{Z}}^{q} \mathbb{Z} \oplus J_{K}\right) ; d t_{j_{1}} \wedge \cdots \wedge d t_{j_{q}} \mapsto 1 \otimes \boldsymbol{e}_{j_{1}} \wedge \cdots \wedge \boldsymbol{e}_{j_{q}}$. In particular, $\hat{\Omega}_{{ }_{O_{K_{0}}}^{q}} /\left(p^{n}\right)$ is a free ${ }^{O_{K_{0}}} /\left(p^{n}\right)$-module.

(ii) We have a canonical isomorphism

$$
\left(\bigwedge_{K}^{q} \widehat{\Omega}_{K}^{1}\right)^{\widehat{-}} \rightarrow \widehat{\Omega}_{K}^{q}
$$

(iii) Let $L$ be a finite extension over the completion of an unramified extension of $K$. Then, we have a canonical isomorphism

$$
L \otimes_{K} \hat{\Omega}_{K}^{q} \rightarrow \widehat{\Omega}_{L}^{q} .
$$

Proof. The assertions (i) and (ii) follow from [Hyodo 1986, Lemma (4.4), Remark 3] respectively. The canonical exact sequence

$$
0 \rightarrow{O_{L}}_{L} \otimes_{O_{K}} \Omega_{\mathscr{O}_{K} / \mathbb{Z}}^{1} \rightarrow \Omega_{\mathscr{O}_{L} / \mathbb{Z}}^{1} \rightarrow \Omega_{\mathscr{O}_{L} / \mathscr{O}_{K}}^{1} \rightarrow 0
$$

(from [Scholl 1998, Section 3.4, footnote]) induces the exact sequence

$$
\Omega_{\mathscr{O}_{L} / \mathscr{O}_{K}}^{1}\left[p^{n}\right] \rightarrow{O_{L}}_{L} \otimes_{\mathscr{O}_{K}} \Omega_{\mathscr{O}_{K} / \mathbb{Z}}^{1} /\left(p^{n}\right) \stackrel{\alpha_{n}}{\rightarrow} \Omega_{\mathscr{O}_{L} / \mathbb{Z}}^{1} /\left(p^{n}\right) \rightarrow \Omega_{\mathscr{O}_{L} / \mathscr{O}_{K}}^{1} /\left(p^{n}\right) \rightarrow 0,
$$

where $\Omega_{\mathscr{O}_{L} / \mathscr{O}_{K}}^{1}\left[p^{n}\right]$ denotes the kernel of the multiplication by $p^{n}$ on $\Omega_{\mathscr{O}_{L} / \mathscr{C}_{K}}^{1}$. Fix $c \in \mathbb{N}$ such that $p^{c} \Omega_{{ }_{O_{L}} / \mathscr{O}_{K}}^{1}=0$. Then, the kernel and cokernel of $\alpha_{n}$ are killed by $p^{c}$. Denote by $2_{n}$ and $Q_{n}$ the kernel of the canonical maps

$$
\begin{aligned}
& \otimes_{\mathscr{O}_{L}}^{q}\left(\mathscr{O}_{L} \otimes_{\mathscr{O}_{K}} \Omega_{\mathscr{O}_{K} / \mathbb{Z}}^{1} /\left(p^{n}\right)\right) \rightarrow \wedge_{\mathscr{O}_{L}}^{q}\left(\bigcirc_{L} \otimes_{O_{K}} \Omega_{\mathscr{O}_{K} / \mathbb{Z}}^{1} /\left(p^{n}\right)\right), \\
& \otimes_{\mathscr{O}_{L}}^{q} \Omega_{\mathscr{O}_{L} / \mathbb{Z}}^{1} /\left(p^{n}\right) \rightarrow \Omega_{\mathscr{O}_{L} / \mathbb{Z}}^{q} /\left(p^{n}\right) .
\end{aligned}
$$


We consider the commutative diagram

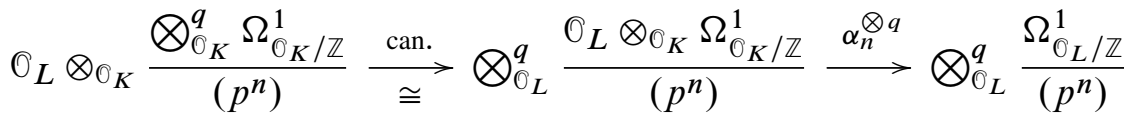

$$
\begin{aligned}
& \Downarrow \text { can. } \quad \forall \text { can. } \quad \forall \text { can. } \\
& 0_{L} \otimes_{\mathscr{O}_{K}} \frac{\Omega_{\mathscr{O}_{K} / \mathbb{Z}}^{q}}{\left(p^{n}\right)} \stackrel{\text { can. }}{\cong} \bigwedge_{\mathscr{O}_{L}}^{q} \frac{\mathscr{O}_{L} \otimes_{\mathscr{O}_{K}} \Omega_{\mathscr{O}_{K} / \mathbb{Z}}^{1}}{\left(p^{n}\right)} \stackrel{\bigwedge^{q} \alpha_{n}}{\longrightarrow} \frac{\Omega_{\mathscr{O}_{L} / \mathbb{Z}}^{q}}{\left(p^{n}\right)} .
\end{aligned}
$$

We have only to prove that the kernel and cokernel of $\bigwedge^{q} \alpha_{n}$ are killed by $p^{3 q c}$. Indeed, if this is true, then we decompose the canonical map

$$
\alpha_{n}^{q}: \bigcirc_{L} \otimes_{\mathscr{O}_{K}} \Omega_{\mathscr{O}_{K} / \mathbb{Z}}^{q} /\left(p^{n}\right) \rightarrow \Omega_{\mathscr{O}_{L} / \mathbb{Z}}^{q} /\left(p^{n}\right)
$$

into the following exact sequences:

$$
\begin{gathered}
0 \longrightarrow \operatorname{ker} \alpha_{n}^{q} \stackrel{\text { inc. }}{\longrightarrow} \mathscr{O}_{L} \otimes_{\mathscr{O}_{K}} \Omega_{\mathscr{O}_{K} / \mathbb{Z}}^{q} /\left(p^{n}\right) \stackrel{\alpha_{n}^{q}}{\longrightarrow} \operatorname{Im} \alpha_{n}^{q} \longrightarrow 0, \\
0 \longrightarrow \operatorname{Im} \alpha_{n}^{q} \stackrel{\text { inc. }}{\longrightarrow} \Omega_{{ }_{O_{L}} / \mathbb{Z}}^{q} /\left(p^{n}\right) \stackrel{\text { pr. }}{\longrightarrow} \operatorname{cok} \alpha_{n}^{q} \longrightarrow 0 .
\end{gathered}
$$

By passing to limits, we obtain the following exact sequences:

$0 \longrightarrow \lim _{n} \operatorname{ker} \alpha_{n}^{q} \stackrel{\text { inc. }}{\longrightarrow} \mathcal{O}_{L} \otimes_{0_{K}} \hat{\Omega}_{\mathscr{O}_{K}}^{q} \stackrel{\text { can. }}{\longrightarrow} \lim _{\longleftarrow} \operatorname{Im} \alpha_{n}^{q} \stackrel{\delta}{\longrightarrow} \lim _{n}^{1} \operatorname{ker} \alpha_{n}^{q}$,

$$
0 \longrightarrow \lim _{\longleftarrow} \operatorname{Im} \alpha_{n}^{q} \stackrel{\text { inc. }}{\longrightarrow} \widehat{\Omega}_{\mathrm{O}_{L}}^{q} \stackrel{\text { pr. }}{\longrightarrow} \lim _{\longleftarrow} \operatorname{cok} \alpha_{n}^{q} .
$$

Since $\operatorname{ker} \alpha_{n}^{q}$ and $\operatorname{cok} \alpha_{n}^{q}$ are killed by $p^{3 q c}, \lim _{n} \operatorname{ker} \alpha_{n}^{q}$ and $\lim _{n}^{1} \operatorname{ker} \alpha_{n}^{q}, \lim _{\leftarrow} \operatorname{cok} \alpha_{n}^{q}$ are also killed by $p^{3 q c}$ [Neukirch et al. 2008, Proposition 2.7.4]. Hence, the kernel and cokernel of the canonical map $0_{L} \otimes_{O_{K}} \hat{\Omega}_{O_{K}}^{q} \rightarrow \hat{\Omega}_{\mathscr{O}_{L}}^{q}$ are killed by $p^{3 q c}$ and $p^{6 q c}$ respectively. By inverting $p$, we obtain the assertion.

Note that the kernel and cokernel of $\alpha_{n}^{\otimes q}$ are killed by $p^{2 q c}$ by Lemma 1.9. By the snake lemma, it suffices to prove that the cokernel of the map $\alpha_{n}^{\otimes q}: \mathscr{2}_{n} \rightarrow Q_{n}$ is killed by $p^{q c}$. The $\mathrm{O}_{L}$-module $Q_{n}$ is generated by the elements of the form $x:=x_{1} \otimes \cdots \otimes x_{q}$ with $x_{i} \in \Omega_{{ }_{O_{L} / \mathbb{Z}}^{1}}^{1} /\left(p^{n}\right)$ such that $x_{i}=x_{j}$ for some $i \neq j$. Since the cokernel of $\alpha_{n}$ is killed by $p^{c}$, there exist $y_{1}, \ldots, y_{q} \in \mathscr{O}_{L} \otimes_{O_{K}} \Omega_{O_{L} / \mathbb{Z}}^{1} /\left(p^{n}\right)$ such that $p^{c} x_{i}=\alpha_{n}\left(y_{i}\right)$ and $y_{i}=y_{j}$. Hence we have $p^{q c} x=\left(p^{c} x_{1}\right) \otimes \cdots \otimes\left(p^{c} x_{q}\right)=$ $\alpha_{n}^{\otimes q}\left(y_{1} \otimes \cdots \otimes y_{q}\right)$ and $y_{1} \otimes \cdots \otimes y_{q} \in 2_{n}$, which implies the assertion.

Remark 1.11. If $\left[k_{K}: k_{K}^{p}\right]=p^{d}<\infty$, then $\operatorname{dim}_{K} \hat{\Omega}_{K}^{q}=\left(\begin{array}{l}d \\ q\end{array}\right)<\infty$ for $q \in \mathbb{N}$ by Lemma 1.10. In particular, the canonical derivation $d$ is $K_{\text {can-linear since the }}$ restriction $\left.d\right|_{K_{\text {can }}}$ factors through $\widehat{\Omega}_{K_{\text {can }}}^{1}=0$ by functoriality. 
Defintion 1.12. Fix a lift $\left\{t_{j}\right\}_{j \in J_{K}} \subset \mathrm{O}_{K_{0}}$ of a $p$-basis of $k_{K}$. By Lemma 1.10(i), $d x$ for $x \in \mathcal{O}_{K_{0}}$ can be uniquely written in the form $\sum_{j \in J_{K}} d t_{j} \otimes \partial_{j}(x)$, where $\left\{\partial_{j}(x)\right\}_{j \in J_{K}} \subset \mathcal{O}_{K_{0}}$ is such that $\left\{v_{p}\left(\partial_{j}(x)\right)\right\}_{j \in J_{K}} \rightarrow \infty$. Note that $\left\{\partial_{j}\right\}_{j \in J_{K}}$ are mutually commutative derivations of $0_{K_{0}}$ by the formula $d_{1} \circ d=0$. We also note that $\partial_{j}$ is continuous since we have the inequality $v_{p}\left(\partial_{j}(x)\right) \geq v_{p}(x)$ for $x \in \mathbb{O}_{K_{0}}$, which we can check by taking modulo $p$.

The following is another characterization of the canonical subfields.

Proposition 1.13 [Brinon 2006, Proposition 2.28]. We have the exact sequence

$$
0 \longrightarrow K_{\text {can }} \stackrel{\text { inc. }}{\longrightarrow} K \stackrel{d}{\longrightarrow} \hat{\Omega}_{K}^{1} .
$$

Proof. We first reduce to the case $K=K_{0}$. In the case that $K$ satisfies condition (H), we obtain the exact sequence by applying $K_{\text {can }} \otimes_{K_{\text {can }, 0}}$ to the exact sequence for $K=K_{0}$ by Remark 1.4(ii) and Lemma 1.10(iii). In the general case, we choose a finite Galois extension $K^{\prime} / K_{\text {can }}$ such that $K K^{\prime}$ satisfies condition (H) by Epp's Theorem 1.6. Since we have $\left(K K^{\prime}\right)_{\text {can }}=K^{\prime}$ by Lemma 1.5 (iv), $K^{\prime} \otimes_{K_{\text {can }}} K=K K^{\prime}$ by Lemma $1.5(\mathrm{i})$ and $\left(\hat{\Omega}_{K K^{\prime}}^{1}\right)^{G_{K^{\prime} / K_{\text {can }}}}=\widehat{\Omega}_{K}^{1}$ by Lemma 1.10 (iii), the assertion follows from Galois descent.

We will prove the assertion in the case $K=K_{0}$. We may replace $K_{\text {can }}, K$ and $\widehat{\Omega}_{K}^{1}$ by $\hat{O}_{K_{\text {can }}}, \mathscr{O}_{K}$ and $\hat{\Omega}_{\mathscr{O}_{K}}^{1}$ respectively. Notation is as above. Let $\varphi$ be the Frobenius on $\hat{O}_{K}$ given by $\varphi\left(t_{j}\right)=t_{j}^{p}$ for $j \in J_{K}$. Let $\varphi_{*}: \hat{\Omega}_{K}^{1} \rightarrow \hat{\Omega}_{K}^{1}$ be the Frobenius induced by $\varphi$. Since we have $d \circ \varphi=\varphi_{*} \circ d$, by a simple calculation, we have $\partial_{j} \circ \varphi=p t_{j}^{p-1} \varphi \circ \partial_{j}$, that is, $\left(t_{j} \partial_{j}\right) \circ \varphi=p \varphi \circ\left(t_{j} \partial_{j}\right)$ for $j \in J_{K}$.

The ring $\varphi\left(\mathbb{O}_{K}\right)$ is a complete discrete valuation ring of mixed characteristic $(0, p)$ and we may regard its residue field as $k_{K}^{p}$. Let $\Lambda:=\{0, \ldots, p-1\} \oplus J_{K}$. Since the image of $\left\{\boldsymbol{t}^{\boldsymbol{n}}\right\}_{\boldsymbol{n} \in \Lambda}$ in $k_{K}$ forms a $k_{K}^{p}$-basis of $k_{K}$, by approximation, every element $x \in \mathfrak{O}_{K}$ can be uniquely written in the form $x=\sum_{\boldsymbol{n} \in \Lambda} \varphi\left(a_{\boldsymbol{n}}\right) \boldsymbol{t}^{\boldsymbol{n}}$, where $a_{\boldsymbol{n}} \in \mathcal{O}_{K}$ is such that $\left\{v_{p}\left(a_{\boldsymbol{n}}\right)\right\}_{\boldsymbol{n} \in \Lambda} \rightarrow \infty$. We claim that if $\varphi^{n}(x) \in \operatorname{ker} d$ with $n \in \mathbb{N}$ and $x \in \mathscr{O}_{K}$, we have $x \in \varphi\left(\mathbb{O}_{K}\right)$. Since the Frobenius $\varphi_{*}$ on $\widehat{\Omega}_{\mathscr{O}_{K}}^{1}$ is injective by Lemma 1.10(i) and the commutativity $d \circ \varphi=\varphi_{*} \circ d$, we may assume $n=0$. By definition, we have $\partial_{j}(x)=0$ for all $j \in J_{K}$. We have

$$
t_{j} \partial_{j}(x)=\sum_{\boldsymbol{n} \in \Lambda}\left(t_{j} \partial_{j} \circ \varphi\right)\left(a_{\boldsymbol{n}}\right) \boldsymbol{t}^{\boldsymbol{n}}+\sum_{\boldsymbol{n} \in \Lambda} \varphi\left(a_{\boldsymbol{n}}\right) t_{j} \partial_{j}\left(\boldsymbol{t}^{\boldsymbol{n}}\right)=\sum_{\boldsymbol{n} \in \Lambda} \varphi\left(p t_{j} \partial_{j}\left(a_{\boldsymbol{n}}\right)+n_{j} a_{\boldsymbol{n}}\right) \boldsymbol{t}^{\boldsymbol{n}} .
$$

Hence, we have $a_{\boldsymbol{n}}=-n_{j}^{-1} p t_{j} \partial_{j}\left(a_{\boldsymbol{n}}\right)$ if $n_{j} \neq 0$. Therefore, for $\boldsymbol{n} \in \Lambda \backslash\{\boldsymbol{0}\}$, we have $v_{p}\left(a_{n}\right) \geq v_{p}\left(a_{n}\right)+1$, that is, $a_{n}=0$, which implies the claim. By using the claim, if we have $x \in \operatorname{ker} d$, then we have $x \in \bigcap_{n \in \mathbb{N}} \varphi^{n}\left(0_{K}\right)$. Since the complete discrete valuation ring $\bigcap_{n \in \mathbb{N}} \varphi^{n}\left(\mathcal{O}_{K}\right)$ is absolutely unramified with residue field $k_{K}^{p^{\infty}}$, the inclusion ${ }^{O_{K}} K_{\text {can }} \subset \bigcap_{n \in \mathbb{N}} \varphi^{n}\left(\mathbb{O}_{K}\right)$ is an equality by approximation, which implies the assertion. 
1D. A spectral sequence of continuous group cohomology. The following lemma is a basic fact when we calculate continuous Galois cohomology whose coefficient is an inverse limit of $p$-adic Banach spaces with surjective transition maps. For example, we need it later when we calculate cohomology of $\mathbb{B}_{\mathrm{dR}}^{+}$-modules.

Lemma 1.14 (Compare [Neukirch et al. 2008, Theorem 2.7.5].). Let $G$ be a profinite group and $\left\{M_{n}\right\}_{n \in \mathbb{N}}$ be an inverse system of continuous $G$-modules (each $M_{n}$ may not be discrete) such that the transition map $M_{n+1} \rightarrow M_{n}$ admits a continuous section (as topological spaces) for all $n \in \mathbb{N}$. Let $M_{\infty}$ be the continuous $G$-module $\lim _{\leftarrow} M_{n}$ with the inverse limit topology. Then, we have a canonical exact sequence

$$
0 \longrightarrow \lim _{n}^{1} H^{q-1}\left(G, M_{n}\right) \longrightarrow H^{q}\left(G, M_{\infty}\right) \longrightarrow \lim _{n} H^{q}\left(G, M_{n}\right) \longrightarrow 0
$$

for all $q \in \mathbb{N}$, where $\lim ^{\bullet}$ is the derived functor of $\lim _{\longleftarrow}$ in the category of inverse systems of abelian groups indexed by $\mathbb{N}$.

Proof. Let $\mathscr{C}_{\infty}^{\bullet}:=\mathscr{C}_{\text {cont. }}^{\bullet}\left(G, M_{\infty}\right)$ (resp. $\left.\mathscr{C}_{n}^{\bullet}:=\mathscr{C}_{\text {cont. }}^{\bullet}\left(G, M_{n}\right)\right)$ be the continuous cochain complex of $G$ with coefficients in $M_{\infty}$ (resp. $M_{n}$ ). Then, $\left\{\mathscr{C}_{n}^{\bullet}\right\}_{n \in \mathbb{N}}$ forms an inverse system of cochain complexes and we have $\mathscr{C}_{\infty}^{\bullet}=\lim _{n} \mathscr{C}_{n}^{\bullet}$. Moreover, the transition maps of the inverse system $\left\{\mathscr{C}_{n}^{\bullet}\right\}_{n \in \mathbb{N}}$ are surjective by the existence of continuous sections, in particular, $\left\{\mathscr{C}_{n}^{\bullet}\right\}_{n \in \mathbb{N}}$ satisfies the Mittag-Leffler condition. Then, the assertion follows from [Weibel 1994, Variant in pp.84].

1E. Hyodo's calculations of Galois cohomology. We will recall Hyodo's calculations of Galois cohomology. For $n \in \mathbb{Z}$, denote by $\mathbb{Z}_{p}(n)$ the $n$-th Tate twist of $\mathbb{Z}_{p}$. For a $\mathbb{Z}_{p}\left[G_{K}\right]$-module $V$, let $V(n):=V \otimes_{\mathbb{Z}_{p}} \mathbb{Z}_{p}(n)$.

Theorem 1.15 [Hyodo 1986, Theorem 1]. For $n \in \mathbb{N}$ and $q \in \mathbb{Z}$, we have canonical isomorphisms

$$
H^{n}\left(G_{K}, \mathbb{C}_{p}(q)\right) \cong \begin{cases}0 & q \neq n, n-1, \\ \hat{\Omega}_{K}^{q} & \text { otherwise. }\end{cases}
$$

We will generalize the following theorem as an application of the Main Theorem in Section 7.

Theorem 1.16. (i) [Hyodo 1986, Theorem 2] We have the exact sequence

$$
0 \longrightarrow H^{1}\left(G_{K_{\text {can }}}, \mathbb{Z}_{p}(1)\right) \stackrel{\text { Inf }}{\longrightarrow} H^{1}\left(G_{K}, \mathbb{Z}_{p}(1)\right) \stackrel{\text { can. }}{\longrightarrow} H^{1}\left(G_{K}, \mathbb{C}_{p}(1)\right) \text {. }
$$

(ii) [Hyodo 1987, Theorem (0-2)] If $k_{K}$ is separably closed, then

$$
\text { Inf : } H^{1}\left(G_{K_{\text {can }}}, \mathbb{Z}_{p}(n)\right) \rightarrow H^{1}\left(G_{K}, \mathbb{Z}_{p}(n)\right)
$$

is an isomorphism for $n \neq 1$. 
1F. Closed subgroups of $\boldsymbol{G}_{\boldsymbol{K}}$. Let $L$ be an algebraic extension of $K$ in $\mathbb{C}_{p}$. Let $\widehat{L}^{\text {alg }}$ be the algebraic closure of $\widehat{L}$ in $\mathbb{C}_{p}$. Let $M$ be a finite extension of $\widehat{L}$ and choose a polynomial $f(X) \in \hat{L}[X]$ such that $M \cong \widehat{L}[X] /(f(X))$. Let $f_{0}(X) \in L[X]$ be a polynomial such that the $p$-adic valuations of the coefficients of $f-f_{0}$ are large enough. Then, we have $M \cong \widehat{L}[X] /\left(f_{0}(X)\right)$ by Krasner's lemma. In particular, the algebraic extension $\left(M \cap L^{\mathrm{alg}}\right) / L$ is dense in $M$. Hence, we have a canonical morphism of profinite groups $G_{L} \rightarrow G_{\widehat{L}}$, which is an isomorphism whose inverse $G_{\widehat{L}} \rightarrow G_{L}$ maps $g$ to $\left.g\right|_{L^{\text {alg }}}$. In the sequel, we will identify $G_{L}$ with $G_{\widehat{L}}$ and we also regard $G_{\widehat{L}}$ as a closed subgroup of $G_{K}$.

1G. Perfection. For a subset $J$ of $J_{K}$, we denote the $p$-adic completion of the field $\bigcup_{n \in \mathbb{N}} K\left(\left\{t_{j}^{p^{-n}}\right\}_{j \in J}\right)$ by $K_{J}$. Then, $K_{J}$ is a complete discrete valuation field of mixed characteristic $(0, p)$ with $e_{K_{J} / K}=1$ and its residue field is isomorphic to $\bigcup_{n \in \mathbb{N}} k_{K}\left(\left\{\bar{t}_{j}^{p^{-n}}\right\}_{j \in J}\right)$. We also denote $K_{J_{K}}$ by $K^{\mathrm{pf}}$, which is referred as a perfection of $K$ since the residue field $k_{K^{\mathrm{pf}}} \cong k_{K}^{\mathrm{pf}}$ of $K^{\mathrm{pf}}$ is perfect. Since we may assume that $\left\{t_{j}\right\}_{j \in J_{K}}$ is contained in $K_{0}$ (Section 1A), we may assume $\left(K_{0}\right)_{J}=$ $\left(K_{J}\right)_{0}$, which is denoted by $K_{J, 0}$ for simplicity.

Let $\mathscr{P}\left(J_{K}\right)$ be the subsets of $J_{K}$ consisting of subsets $J \in J_{K}$ such that $J_{K} \backslash J$ is finite. Note that we have $\left[k_{K_{J}}: k_{K_{J}}^{p}\right]=p^{\left|J_{K} \backslash J\right|}<\infty$ for $J \in \mathscr{P}\left(J_{K}\right)$ since $\left\{\bar{t}_{j}\right\}_{j \in J_{K} \backslash J}$ forms of a $p$-basis of $k_{K_{J}}$. We regard $\mathscr{P}\left(J_{K}\right)$ as an inverse system with respect to the reverse inclusion. Then, we have

$$
K \cong \lim _{J \in \mathscr{P}\left(J_{K}\right)} K_{J}=\bigcap_{J \in \mathscr{P}\left(J_{K}\right)} K_{J}
$$

that is, $K$ is represented by an inverse limit of complete discrete valuation fields, whose residue fields admit a finite $p$-basis. In fact, if we endow $J_{K}$ with a wellorder $\precsim$ by the axiom of choice, then for $J \in \mathscr{P}\left(J_{K}\right)$, the subset

$$
\mathscr{E}_{J}:=\{1\} \cup\left\{\begin{array}{l|l}
t_{j_{1}}^{a_{1} p^{-n_{1}}} \ldots t_{j_{m}}^{a_{m} p^{-n_{m}}} & \begin{array}{l}
j_{1} \precsim \ldots \precsim j_{m} \in J, 0<a_{j_{i}}<p^{n_{j_{i}} \in \mathbb{N}_{>0}} \\
\left(p, a_{j_{i}}\right)=1 \text { for } 1 \leq i \leq m \in \mathbb{N}_{>0}
\end{array}
\end{array}\right\}
$$

of $K_{J}$ forms a basis of $K_{J}$ as a $K$-Banach space. If $J_{1} \subset J_{2}$ are in $\mathscr{P}\left(J_{K}\right)$, then we have $\mathscr{E}_{J_{1}} \subset \mathscr{E}_{J_{2}}$ and the assertion follows from the fact $\{1\}=\bigcap_{J \in \mathscr{P}\left(J_{K}\right)} \mathscr{E}_{J}$.

1H. $G$-regular ring. We will recall basic facts about $G$-regular rings. For details, see [Fontaine 1994b, Section 1].

Let $E$ be a topological field and $G$ a topological group. A finite-dimensional $E$-vector space $V$ is an $E$-representation of $G$ if $V$ has a continuous $E$-linear action of $G$. We denote the category of $E$-representations of $G$ by $\operatorname{Rep}_{E} G$. We call $B$ an $(E, G)$-ring if $B$ is a commutative $E$-algebra and $G$ acts on $B$ by $E$-algebra automorphisms. Let $B$ be an $(E, G)$-ring. For $V \in \operatorname{Rep}_{E} G$, let $D_{B}(V):=\left(B \otimes_{E} V\right)^{G}$ 
and we will call the following canonical homomorphism the comparison map:

$$
\alpha_{B}(V): B \otimes_{B^{G}} D_{B}(V) \rightarrow B \otimes_{E} V .
$$

We say that an $(E, G)$-ring $B$ is $G$-regular if the following is satisfied:

$\left(G \cdot R_{1}\right)$ The ring $B$ is reduced.

$\left(G \cdot R_{2}\right)$ For all $V \in \operatorname{Rep}_{E} G, \alpha_{B}(V)$ is injective.

$\left(G \cdot R_{3}\right)$ Every $G$-stable $E$-line in $B$ is generated by an invertible element of $B$.

Here, a $G$-stable $E$-line in $B$ means one-dimensional $G$-stable $E$-vector space in $B$. The condition $\left(G \cdot R_{3}\right)$ implies that $B^{G}$ is a field. We say that $V \in \operatorname{Rep}_{E} G$ is $B$-admissible if $\alpha_{B}(V)$ is an isomorphism. We denote the category of $B$-admissible $E$-representations of $G$ by $\operatorname{Rep}_{B / E} G$, which is a Tannakian full subcategory of $\operatorname{Rep}_{E} G$ [Fontaine 1994b, Proposition 1.5.2].

Notation. We will call an object of $\operatorname{Rep}_{\mathbb{Q}_{p}} G_{K}$ a $p$-adic representation of $G_{K}$. For a $\left(\mathbb{Q}_{p}, G_{K}\right)$-ring $B$, we denote $\operatorname{Rep}_{B / \mathbb{Q}_{p}} G_{K}$ by $\operatorname{Rep}_{B}^{\text {adm }} G_{K}$ if no confusion arises.

We recall the basic facts about $G$-regular rings.

Lemma 1.17. Let $B$ be a field and $G$ a group acting on $B$ by ring automorphisms. Let $M$ be a finite-dimensional B-vector space with semilinear $G$-action. Then, the canonical map

$$
B \otimes_{B^{G}} M^{G} \rightarrow M
$$

is injective. In particular, we have $\operatorname{dim}_{B G} M^{G} \leq \operatorname{dim}_{B} M$.

Proof. Suppose that the assertion does not hold. Let $n \in \mathbb{N}$ be the smallest integer such that there exist $n$ elements $v_{1}, \ldots, v_{n} \in M^{G}$ which are linearly independent over $B^{G}$ but not over $B$. Let $\sum_{1 \leq i \leq n} \lambda_{i} v_{i}=0$ be a nontrivial relation with $\lambda_{i} \in B$. Since $B$ is a field, we may assume that $\lambda_{1}=1$. Then, we have

$$
0=(g-1)\left(\sum_{1 \leq i \leq n} \lambda_{i} v_{i}\right)=\sum_{1<i \leq n}\left(g\left(\lambda_{i}\right)-\lambda_{i}\right) v_{i} .
$$

Hence, we have $\lambda_{i} \in B^{G}$ by assumption, which is a contradiction.

Example 1.18 [Fontaine 1994b, Proposition 1.6.1]. All ( $E, G)$-rings which are fields are $G$-regular. In fact, we have only to verify $\left(G \cdot R_{2}\right)$, which follows by applying the above lemma to $M:=B \otimes_{E} V$.

Lemma 1.19 [Fontaine 1994b, Proposition 1.4.2]. Let B be a $G$-regular $(E, G)$ ring and $V$ an E-representation of $G$. Then, we have $\operatorname{dim}_{B} D_{B}(V) \leq \operatorname{dim}_{E} V$. Moreover, the equality holds if and only if $V$ is $B$-admissible. 
Lemma 1.20 [Fontaine 1994b, Proposition 1.6.5]. Let B be a $G$-regular $(E, G)$ ring and $B^{\prime}$ an $E$-subalgebra of $B$ stable by $G$. Assume that $B^{\prime}$ satisfies $\left(G \cdot R_{3}\right)$ and that the canonical map $B^{G} \otimes_{B^{\prime} G} B^{\prime} \rightarrow B$ is injective. Then, $B^{\prime}$ is a $G$ regular $(E, G)$-ring. Moreover, if $V \in \operatorname{Rep}_{E} G$ is $B^{\prime}$-admissible, then $V$ is $B$ admissible and the canonical map

$$
B^{G} \otimes_{B^{\prime} G} D_{B^{\prime}}(V) \rightarrow D_{B}(V)
$$

is an isomorphism.

Lemma 1.21 [Fontaine 1994b, Corollaire 1.6.6]. Let $B^{\prime}$ be an integral domain which is an $(E, G)$-ring, and $B$ the fraction field of $B^{\prime}$. If $B^{\prime}$ satisfies $\left(G \cdot R_{3}\right)$ and $B^{\prime G}=B^{G}$, then $B^{\prime}$ is $G_{K}$-regular.

Remark 1.22 (restriction). Let $B$ be a $G$-regular $(E, G)$-ring and $H$ a subgroup of $G$ such that $B$ is $H$-regular as an $(E, H)$-ring. If $V \in \operatorname{Rep}_{E} G$ is $B$-admissible, then $\left.V\right|_{H}$ is also $B$-admissible in $\operatorname{Rep}_{E} H$. Moreover, we have a canonical isomorphism $B^{H} \otimes_{B^{G}} D_{B}(V) \cong D_{B}\left(\left.V\right|_{H}\right)$. Indeed, the admissibility of $V$ implies that we have the comparison isomorphism $B \otimes_{B^{G}} D_{B}(V) \cong B \otimes_{E} V$ as $B\left[G_{K}\right]$ modules. By taking $H$-invariants, we have $B^{H} \otimes_{B^{G}} D_{B}(V) \cong D_{B}\left(\left.V\right|_{H}\right)$. In particular, we have $\operatorname{dim}_{B^{H}} D_{B}\left(\left.V\right|_{H}\right)=\operatorname{dim}_{B^{G}} D_{B}(V)=\operatorname{dim}_{E} V$, which implies the $B$-admissibility of $\left.V\right|_{H}$ by Lemma 1.19 .

\section{A generalization of Sen's theorem}

The aim of this section is to prove the following generalization of Sen's theorem on $\mathbb{C}_{p}$-admissible representations [Sen 1980, Corollary in (3.2)].

Theorem 2.1. Let $V \in \operatorname{Rep}_{\mathbb{Q}_{p}} G_{K}$. The following are equivalent:

(i) There exists a finite extension $L$ over the maximal unramified extension of $K$ such that $G_{L}$ acts trivially on $V$.

(ii) $V$ is $\mathbb{C}_{p^{-} \text {-admissible. }}$

(iii) $\left.V\right|_{K^{\mathrm{pf}}}$ is $\mathbb{C}_{p^{-}}$admissible as an object of $\operatorname{Rep}_{\mathbb{Q}_{p}} G_{K^{\mathrm{pf}}}$.

Lemma 2.2. Let $E$ be a field of characteristic 0 and $\rho: U_{\mathbb{Q}_{p}}^{(n)} \ltimes \prod_{i \in I} p^{n_{i}} \mathbb{Z}_{p} \rightarrow$ $\mathrm{GL}_{r}(E)$ a group homomorphism with $n, r \in \mathbb{N}_{>0}$ and $\left(n_{i}\right)_{i \in I} \in \mathbb{N}^{I}$, where the action of $U_{\mathbb{Q}_{p}}^{(n)}$ on $\prod_{i \in I} p^{n_{i}} \mathbb{Z}_{p}$ is given by scalar multiplication. If $\operatorname{ker} \rho$ contains an open subgroup of $U_{\mathbb{Q} p}^{(n)}$, then the image of $\rho$ is finite.

Proof. By shrinking $U_{\mathbb{Q}_{p}}^{(n)}$, we may assume that $\operatorname{ker} \rho$ contains $U_{\mathbb{Q}_{p}}^{(n)}$. Also, we may assume that $E$ is algebraically closed. Let $x_{0}:=1+p^{n} \in U_{\mathbb{Q} p}^{(n)}, \boldsymbol{x} \in \prod_{i \in I} p^{n_{i}} \mathbb{Z}_{p}$. By the fact that $\operatorname{ker} \rho$ is a normal subgroup of $U:=U_{\mathbb{Q}_{p}}^{(n)} \ltimes \prod_{i \in I} p^{n_{i}} \mathbb{Z}_{p}$ and a simple calculation, we have

$$
(1, \boldsymbol{x})^{-1}\left(x_{0}, \mathbf{0}\right)(1, \boldsymbol{x})\left(x_{0}^{-1}, \mathbf{0}\right)=\left(1,\left(x_{0}-1\right) \boldsymbol{x}\right)=\left(1, p^{n} \boldsymbol{x}\right) \in \operatorname{ker} \rho .
$$


In particular, $\operatorname{ker} \rho$ contains $U_{\mathbb{Q}_{p}}^{(n)} \ltimes \prod_{i \in I} p^{n+n_{i}} \mathbb{Z}_{p}$ as a normal subgroup. By taking the quotient of $U$ by this subgroup, $\rho$ factors through a group homomorphism $\bar{\rho}:\left(\mathbb{Z} / p^{n} \mathbb{Z}\right)^{I} \rightarrow \mathrm{GL}_{r}(E)$.

To prove the assertion, it suffices to prove that for any finite subset $S$ of $\operatorname{Im} \bar{\rho}$, we have $|S| \leq p^{n r}$. Any $g \in \operatorname{Im} \bar{\rho}$ is conjugate to a diagonal matrix whose diagonal entries are in $\mu_{p^{n}}(E)$ since the order of $g$ divides $p^{n}$. Since the elements of $S$ commute, $S$ is simultaneously diagonalizable. Hence, up to conjugation, $S$ is contained in the set $\left\{\operatorname{diag}\left(a_{1}, \ldots, a_{r}\right) \mid a_{i} \in \mu_{p^{n}}(E)\right\}$, whose order is $p^{n r}$.

Proof of Theorem 2.1. The implication (i) $\Rightarrow$ (ii) follows from Hilbert 90 and (ii) $\Rightarrow$ (iii) follows from Remark 1.22. We will prove (iii) $\Rightarrow$ (i). Note that if $k_{K}$ is perfect, then the assertion is a theorem of Sen ([1980, Corollary in (3.2)]).

By replacing $K$ by a finite extension of $K^{\text {ur }}$, we may assume that $k_{K}$ is separably closed and $K$ satisfies condition (H). In this case, the assertion to prove is that $G_{K}$ acts on $V$ via a finite quotient. Since the residue field $k_{K}^{\mathrm{pf}}$ of $K^{\mathrm{pf}}$ is algebraically closed, $G_{K^{\text {pf }}}=G_{K^{\text {geo }}}$ acts on $V$ via a finite quotient by Sen's theorem, where $K^{\text {geo }}:=\bigcup_{n \in \mathbb{N}} K\left(\left\{t_{j}^{p^{-n}}\right\}_{j \in J_{K}}\right)$. Hence, there exists a finite extension $L / K$ such that $G_{L K^{\text {geo }}}$ acts trivially on $V$. In particular, if we put $K_{\infty}:=K^{\mathrm{geo}}\left(\mu_{p} \infty\right)$, then $G_{L K_{\infty}}$ acts trivially on $V$. In the following, we regard $V$ as a $p$-adic representation of $G_{L K_{\infty} / L}$. Take a basis of $V$ and let $\rho^{\prime}: G_{L K_{\infty} / L} \rightarrow \mathrm{GL}_{r}\left(\mathbb{Q}_{p}\right)$ be the corresponding matrix presentation of $V$ with $r:=\operatorname{dim}_{\mathbb{Q}_{p}} V$. We have only to prove that the image of $\rho^{\prime}$ is finite.

Since $K$ satisfies condition (H), we have an isomorphism $G_{K_{\infty} / K} \cong U_{\mathbb{Q}_{p}}^{\left(n_{0}\right)} \ltimes \mathbb{Z}_{p}^{J_{K}}$, where $n_{0} \in \mathbb{N}_{>1}$ satisfies $G_{K}\left(\mu_{p} \infty\right) / K \cong U_{\mathbb{Q}_{p}}^{\left(n_{0}\right)}$ via the cyclotomic character and $U_{\mathbb{Q}_{p}}^{\left(n_{0}\right)}$ acts on $\mathbb{Z}_{p}^{J_{K}}$ by scalar multiplication (see [Hyodo 1986, Section 1] for details). We have $G_{L K_{\infty} / L K}$ geo $\leq \operatorname{ker} \rho^{\prime} \unlhd_{c} G_{L K_{\infty} / L}$. By using the restriction map $\operatorname{Res}_{K_{\infty}}^{L K_{\infty}}$ and the above isomorphism, we may regard these groups as subgroups of $U_{\mathbb{Q}_{p}}^{\left(n_{0}\right)} \ltimes \mathbb{Z}_{p}^{J_{K}}$. Since $G_{L K_{\infty} / L}$ is an open subgroup of $G_{K_{\infty} / K}$, there exists $n \in \mathbb{N}$ and $\left(n_{j}\right)_{j \in J_{K}} \in \mathbb{N}^{J_{K}}$ such that $G_{L K_{\infty} / L}$ contains $U:=U_{\mathbb{Q}_{p}}^{(n)} \ltimes \prod_{j \in J_{K}} p^{n_{j}} \mathbb{Z}_{p}$ as an open subgroup. Since $G_{L K_{\infty} / L K^{\text {geo }}}$ is an open subgroup of $G_{K_{\infty} / K^{\text {geo }}} \cong$ $G_{K\left(\mu_{p} \infty\right) / K} \cong U_{\mathbb{Q}_{p}}^{\left(n_{0}\right)} \cong \mathbb{Z}_{p}$, ker $\rho^{\prime}$ contains an open subgroup of $U_{\mathbb{Q}_{p}}^{(n)}$. Therefore, the group homomorphism $\rho:=\left.\rho^{\prime}\right|_{U}: U \rightarrow \operatorname{GL}_{r}\left(\mathbb{Q}_{p}\right)$ satisfies the assumption of Lemma 2.2, hence, the image of $\rho$ is finite. Since $U$ is open in $G_{L K_{\infty} / L}$, we obtain the assertion.

\section{Basic construction of rings of $p$-adic periods}

Throughout this section, let $\mathscr{K}$ be a closed subfield of $\mathbb{C}_{p}$ whose value group $v_{p}\left(\mathscr{K}^{\times}\right)$ is discrete. We will recall the construction of rings of $p$-adic periods

$$
\mathbb{A}_{\text {inf }, \mathbb{C}_{p} / \mathscr{K}}, \quad \mathbb{B}_{\text {cris }, \mathbb{C}_{p} / \mathscr{K}}, \quad \mathbb{B}_{\mathrm{st}, \mathbb{C}_{p} / \mathscr{K}}, \quad \mathbb{B}_{\mathrm{dR}, \mathbb{C}_{p} / \mathscr{K}}, \quad \mathbb{B}_{\mathrm{HT}, \mathbb{C}_{p} / \mathscr{K}}
$$


due to Fontaine [1994a], which is functorial with respect to $\mathbb{C}_{p}$ and $\mathscr{K}$. We also recall abstract algebraic properties of these rings as in [Brinon 2006]. Although we do not assume $\mathscr{K}=K$, standard techniques of proofs in the case $\mathscr{K}=K$, which are developed in [Fontaine 1994a; Brinon 2006], can be applied to our situation.

\section{A. Universal pro-infinitesimal thickenings.}

Defintion 3.1 [Fontaine 1994a, Section 1]. A $p$-adically formal pro-infinitesimal $\mathrm{O}_{\mathscr{K}}$-thickening of $\mathbb{O}_{\mathbb{C}_{p}}$ is a pair $\left(D, \theta_{D}\right)$, where

- $D$ is an $O_{\mathscr{K}}$-algebra,

- $\theta_{D}: D \rightarrow \mathscr{O}_{\mathbb{C}_{p}}$ is a surjective $\mathscr{O}_{\mathscr{K}}$-algebra homomorphism such that $D$ is $\left(p, \operatorname{ker} \theta_{D}\right)$-adic Hausdorff complete.

Obviously, $p$-adically formal $\mathscr{O}_{\mathscr{K}}$-thickenings of $\mathbb{O}_{\mathbb{C}_{p}}$ form a category.

Theorem 3.2 [Fontaine 1994a, Théorème 1.2.1]. The category of p-adically formal pro-infinitesimal $\mathscr{O}_{\mathscr{K}}$-thickenings of $\mathbb{O}_{\mathbb{C}_{p}}$ admits a universal object, that is, an initial object.

Such an object is unique up to a canonical isomorphism and we denote it by $\left(\mathbb{A}_{\mathrm{inf}, \mathbb{C}_{p} / \mathscr{K}}, \theta_{\mathbb{C}_{p} / \mathscr{K}}\right)$. Note that $\mathbb{A}_{\text {inf, } \mathbb{C}_{p} / \mathscr{K}}$ is functorial with respect to $\mathbb{C}_{p}$ and $\mathscr{K}$. We recall the construction. Let $R_{\mathbb{C}_{p}}:=\lim _{x \mapsto x^{p}} \mathcal{O}_{\mathbb{C}_{p}} / p \mathscr{O}_{\mathbb{C}_{p}}$ be the perfection of the ring $\mathbb{O}_{\mathbb{C}_{p}} / p \mathscr{O}_{\mathbb{C}_{p}}$. We have the canonical isomorphism

$$
\lim _{x \mapsto x^{p}} \mathscr{O}_{\mathbb{C}_{p}} \rightarrow R_{\mathbb{C}_{p}} ; \quad\left(x^{(n)}\right)_{n \in \mathbb{N}} \mapsto\left(x^{(n)} \bmod p \mathscr{O}_{\mathbb{C}_{p}}\right)_{n \in \mathbb{N}},
$$

where the addition and the multiplication of the LHS are given by

$$
\left(\left(x^{(n)}\right)+\left(y^{(n)}\right)\right)_{n}=\lim _{m}\left(x^{(n+m)}+y^{(n+m)}\right)^{p^{m}}, \quad\left(x^{(n)}\right) \cdot\left(y^{(n)}\right)=\left(x^{(n)} y^{(n)}\right) .
$$

Let $\theta_{\mathbb{C}_{p} / \mathbb{Q}_{p}}: W\left(R_{\mathbb{C}_{p}}\right) \rightarrow \mathbb{O}_{\mathbb{C}_{p}}$ be defined by $\sum_{n \in \mathbb{N}} p^{n}\left[x_{n}\right] \mapsto \sum_{n \in \mathbb{N}} p^{n} x_{n}^{(0)}$. This is a surjective $\mathbb{Z}_{p}$-algebra homomorphism. Let $\theta_{\mathbb{C}_{p} / \mathscr{K}}: \mathscr{O}_{\mathscr{K}} \otimes_{\mathbb{Z}} W\left(R_{\mathbb{C}_{p}}\right) \rightarrow \mathbb{O}_{\mathbb{C}_{p}}$

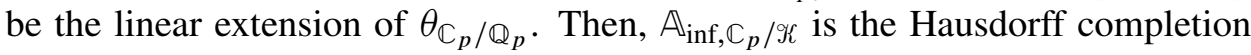
of $\mathscr{O}_{\mathscr{K}} \otimes_{\mathbb{Z}} W\left(R_{\mathbb{C}_{p}}\right)$ with respect to the $\left(p, \operatorname{ker} \theta_{\mathbb{C}_{p} / \mathscr{K}}\right)$-adic topology. We will give an explicit description of $\mathbb{A}_{\text {inf, }}, \mathbb{C}_{p} / \mathscr{K}$ later: Note that the description, together with the isomorphism $W\left(R_{\mathbb{C}_{p}}\right) \cong \mathbb{A}_{\mathrm{inf}, \mathbb{C}_{p} / \mathbb{Q}_{p}}$ (Remark 3.5), immediately implies that $\mathbb{A}_{\text {inf, } \mathbb{C}_{p} / \mathscr{K}}$ is an integral domain (at least) when we have $\mathscr{K}=\mathscr{K}_{0}$.

We define $\tilde{t}_{j}:=\left(t_{j}, t_{j}^{p^{-1}}, \ldots\right) \in R_{\mathbb{C}_{p}}$ and $u_{j}:=t_{j}-\left[\tilde{t}_{j}\right] \in \operatorname{ker} \theta_{\mathbb{C}_{p} / \mathscr{K}_{0}}$. Let $v_{\text {inf, }, \mathbb{C}_{p} / \mathscr{K}}$ be the $p$-adic semivaluation of $\mathbb{A}_{\text {inf }, \mathbb{C}_{p} / \mathscr{K}}$. We put

$$
\begin{aligned}
& \mathbb{A}_{\text {inf, } \mathbb{C}_{p} / \mathbb{Q}_{p}}\left\{\mathbf{u}_{J_{\mathscr{K}}}\right\}:=
\end{aligned}
$$

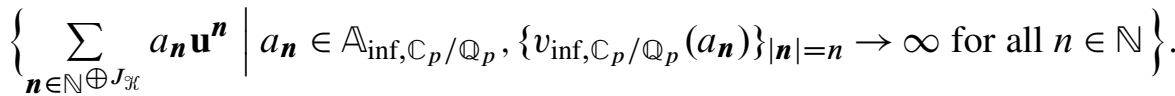


If $J_{\mathscr{K}}$ is finite, $\mathbb{A}_{\text {inf }, \mathbb{C}_{p} / \mathbb{Q}_{p}}\left\{\mathbf{u}_{J_{\mathscr{K}}}\right\}$ is a ring of formal power series with coefficients

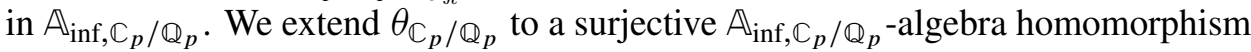

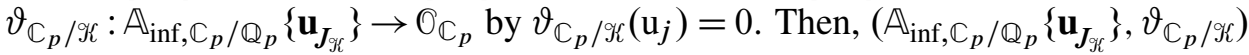
is a $p$-adically formal $\mathbb{Z}_{p}$-pro-infinitesimal thickening of $\mathbb{O}_{\mathbb{C}_{p}}$. We have a canonical $\mathbb{A}_{\text {inf, } \mathbb{C}_{p} / \mathbb{Q}_{p}}$-algebra homomorphism

$$
\iota_{\text {inf, }, \mathbb{C}_{p} / \mathscr{K}}: \mathbb{A}_{\mathrm{inf}, \mathbb{C}_{p} / \mathbb{Q}_{p}}\left\{\mathbf{u}_{J_{\mathscr{H}}}\right\} \rightarrow \mathbb{A}_{\mathrm{inf}, \mathbb{C}_{p} / \mathscr{K}} ; \quad \mathbf{u}^{\boldsymbol{n}} \mapsto \boldsymbol{u}^{\boldsymbol{n}} .
$$

Lemma 3.3. If we assume $\mathscr{K}=\mathscr{K}_{0}$, then $\iota_{\mathrm{inf}, \mathbb{C}_{p} / \mathscr{K}}$ is an isomorphism. In particular, we have

$$
v_{\text {inf, } \mathbb{C}_{p} / \mathscr{K}}(x)=\inf _{\boldsymbol{n} \in \mathbb{N} \oplus J_{\mathscr{K}}} v_{\text {inf, } \mathbb{C}_{p} / \mathbb{Q}_{p}}\left(a_{\boldsymbol{n}}\right)
$$

for $x=\sum_{\boldsymbol{n} \in \mathbb{N} \oplus J_{\mathscr{K}}} a_{\boldsymbol{n}} \boldsymbol{u}^{\boldsymbol{n}}$ with $a_{\boldsymbol{n}} \in \mathbb{A}_{\mathrm{inf}, \mathbb{C}_{p} / \mathbb{Q}_{p}}$.

Proof. Denote $\mathscr{A}=\mathbb{A}_{\mathrm{inf}, \mathbb{C}_{p} / \mathbb{Q}_{p}}\left\{\mathbf{u}_{J_{\mathscr{K}}}\right\}$ and $\vartheta=\vartheta_{\mathbb{C}_{p} / \mathscr{K}}$. We regard $\bigcirc_{\mathscr{K}}$ as a $\mathbb{Z}\left[T_{j}\right]_{j \in J_{\mathscr{T}}}{ }^{-}$ algebra as in Section $1 \mathrm{~A}$. We recall that since $\mathscr{K}=\mathscr{K}_{0}$, the map $\mathbb{Z}\left[T_{j}\right]_{j \in J_{\mathscr{K}}} \rightarrow \mathcal{O}_{\mathscr{K}}$ is formally étale for the $p$-adic topology. We also regard $\mathscr{A}$ as a $\mathbb{Z}\left[T_{j}\right]_{j \in J_{\mathscr{K}}}{ }^{-}$ algebra by $T_{j} \mapsto\left[\tilde{t}_{j}\right]+\mathrm{u}_{j}$. Then, by the lifting property, we can lift the canonical $O_{\mathscr{K}}$-algebra structure on $\mathscr{A} /(p, \operatorname{ker} \vartheta) \cong \mathbb{O}_{\mathbb{C}_{p}} /(p)$ to an $\mathscr{O}_{\mathscr{K}}$-algebra structure on $\mathscr{A} \cong \lim _{n} \mathscr{A} /(p, \operatorname{ker} \vartheta)^{n}:$

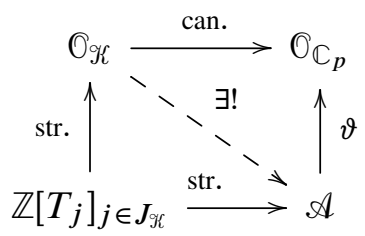

By this structure map, we may regard $\mathscr{A}$ as a pro-infinitesimal $\mathscr{O}_{\mathscr{K}}$-thickening

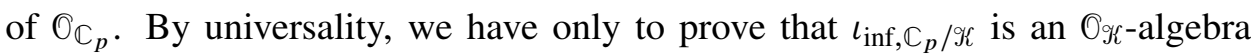
homomorphism. Let $\alpha: \mathscr{O}_{\mathscr{K}} \rightarrow \mathbb{A}_{\mathrm{inf}, \mathbb{C}_{p} / \mathscr{K}}$ be the composition of the structure

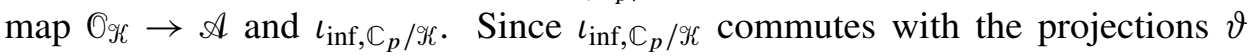
and $\theta_{\mathbb{C}_{p} / \mathscr{K}}$, we have the commutative diagram

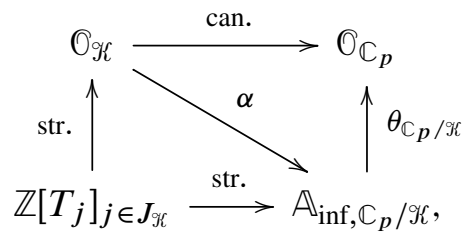

where the horizontal structure map is given by $T_{j} \mapsto t_{j}$. By this diagram and the lifting property, $\alpha$ coincides with the structure map $\mathscr{O}_{\mathscr{K}} \rightarrow \mathbb{A}_{\text {inf, } \mathbb{C}_{p} / \mathscr{K}}$ modulo $\left(p, \operatorname{ker} \theta_{\mathbb{C}_{p} / \mathscr{K}}\right)^{n}$ for all $n \in \mathbb{N}$. Since $\mathbb{A}_{\mathrm{inf}, \mathbb{C}_{p} / \mathscr{K}}$ is $\left(p, \operatorname{ker} \theta_{\mathbb{C}_{p} / \mathscr{K}}\right)$-adically Hausdorff complete, $\alpha$ coincides with the structure map $\mathscr{O}_{\mathscr{K}} \rightarrow \mathbb{A}_{\text {inf, } \mathbb{C}_{p} / \mathscr{K}}$, which implies the assertion. 
For general $\mathscr{K}$, we have:

Lemma 3.4. (i) The canonical map

$$
\mathbb{A}_{\text {inf, } \mathbb{C}_{p} / \mathscr{K}} \rightarrow \mathbb{A}_{\text {inf, } \mathbb{C}_{p} / \mathscr{F} \text { ur }}
$$

is an isomorphism.

(ii) If $\mathscr{L} / \mathscr{K}$ is a finite extension with $\left[k_{\mathscr{L}}: k_{\mathscr{K}}\right]_{\mathrm{sep}}=1$, then the canonical map

$$
\mathrm{O}_{\mathscr{L}} \otimes_{\mathcal{O}_{\mathscr{K}}} \mathbb{A}_{\mathrm{inf}, \mathbb{C}_{p} / \mathscr{K}} \rightarrow \mathbb{A}_{\mathrm{inf}, \mathbb{C}_{p} / \mathscr{L}}
$$

is an isomorphism.

(iii) Let $\mathscr{L}$ be a finite extension of the p-adic completion of an unramified extension of $\mathscr{K}$. Then, the canonical map

$$
\mathbb{A}_{\mathrm{inf}, \mathbb{C}_{p} / \mathscr{K}}\left[p^{-1}\right] /\left(\operatorname{ker} \theta_{\mathbb{C}_{p} / \mathscr{K}}\right)^{n} \rightarrow \mathbb{A}_{\mathrm{inf}, \mathbb{C}_{p} / \mathscr{L}}\left[p^{-1}\right] /\left(\operatorname{ker} \theta_{\mathbb{C}_{p} / \mathscr{L}}\right)^{n}
$$

is an isomorphism for all $n \in \mathbb{N}$.

Proof. (i) The assertion is equivalent to saying that the category of $p$-adically formal Oभr-pro-infinitesimal thickening of $\mathbb{O}_{\mathbb{C}_{p}}$ is equivalent to the category of $p$-adically formal $\mathscr{O}_{\mathscr{Y}}$ ur-pro-infinitesimal thickening of $\mathcal{O}_{\mathbb{C}_{p}}$. Let $\left(D, \theta_{D}\right)$ be a $p$-adically formal Oभ-pro-infinitesimal thickening of $\mathbb{O}_{\mathbb{C}_{p}}$. Then, we have only to prove that there

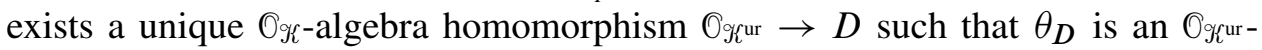
algebra homomorphism. By dévissage, we may replace $D$ by $D /\left(p, \operatorname{ker} \theta_{D}\right)^{n}$ with $n \in \mathbb{N}$. Since $\theta_{D}$ induces an isomorphism $D /\left(p, \operatorname{ker} \theta_{D}\right) \cong \mathbb{O}_{\mathbb{C}_{p}} /(p)$ and $\mathscr{O}_{\mathscr{Y}}$ ur $/ \mathscr{O}_{\mathscr{K}}$ is $p$-adically formally étale, the assertion follows from the commutative diagram

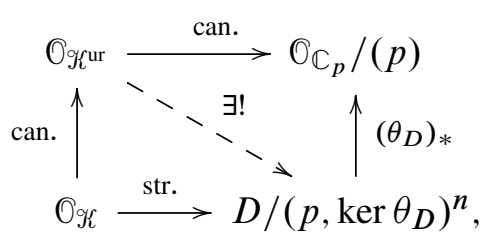

where $\left(\theta_{D}\right)_{*}$ is the ring homomorphism induced by $\theta_{D}$.

(ii) By assumption, the canonical map $\mathscr{O}_{\mathscr{L}} \otimes_{O_{K}} \mathrm{O}_{\mathscr{Y}}$ ur $\rightarrow \mathrm{O}_{\mathscr{L} \text { ur }}$ is an isomorphism. By using this fact and (i), we may assume that $\mathscr{K}=\mathscr{K} \mathcal{K}^{\mathrm{ur}}$ and $\mathscr{L}=\mathscr{L}^{\mathrm{ur}}$. In particular, we may consider the case that $k_{\mathscr{K}}$ is separably closed, where the condition $\left[k_{\mathscr{L}}: k_{\mathscr{K}}\right]_{\mathrm{sep}}=1$ is always satisfied. By faithfully flat descent, the assertion is reduced to the case that $\mathscr{L} / \mathscr{K}$ is Galois. Since $\mathscr{L} / \mathscr{K}$ is a solvable extension [Fesenko and Vostokov 2002, Exercise 2, Section 2, Chapter II], we may assume that $\mathscr{L} / \mathscr{K}$ has prime degree.

By universality, we have only to prove that the LHS is a $p$-adically formal $\mathrm{O}_{\mathscr{L}}$-proinfinitesimal thickening of $\mathscr{O}_{\mathbb{C}_{p}}$. Hence, it suffices to verify that $\mathscr{O}_{\mathscr{L}} \otimes_{\mathscr{O}_{\mathscr{K}}} \mathbb{A}_{\text {inf, } \mathbb{C}_{p} / \mathscr{K}}$ is $(p, I)$-adically Hausdorff complete, where $I$ denotes the kernel of the canonical map $1 \otimes \theta_{\mathbb{C}_{p} / \mathscr{K}}: \mathscr{O}_{\mathscr{L}} \otimes_{\mathscr{O}_{\mathscr{K}}} \mathbb{A}_{\mathrm{inf}, \mathbb{C}_{p} / \mathscr{K}} \rightarrow \mathbb{O}_{\mathbb{C}_{p}}$. Since we have an isomorphism 


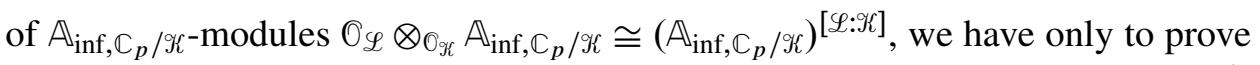
that the topologies on $\mathscr{O}_{\mathscr{L}} \otimes_{\mathcal{O}_{\mathscr{K}}} \mathbb{A}_{\mathrm{inf}, \mathbb{C}_{p} / \mathscr{K}}$ defined by the ideals $(p, I)$ and $\left(p, I^{\prime}\right)$ are equivalent, where $I^{\prime}$ denotes the ideal of $\mathbb{O}_{\mathscr{L}} \otimes_{\mathscr{O}_{\mathscr{K}}} \mathbb{A}_{\mathrm{inf}, \mathbb{C}_{p} / \mathscr{K}}$ generated by $\operatorname{ker}\left(\theta_{\mathbb{C}_{p} / \mathscr{K}}: \mathbb{A}_{\text {inf, } \mathbb{C}_{p} / \mathscr{K}} \rightarrow \mathbb{O}_{\mathbb{C}_{p}}\right)$. By definition, we have $\left(p, I^{\prime}\right) \subset(p, I)$. We have only to prove that we have $I^{n} \subset\left(\pi_{\mathscr{K}} \otimes 1, I^{\prime}\right)$ for some $n \in \mathbb{N}$ since $p$ divides $\pi_{\mathscr{K}}^{e_{\mathscr{C}}}$.

In the following, for $x \in \mathbb{O}_{\mathbb{C}_{p}}$, we denote by $\tilde{x}$ any element $\tilde{x} \in R_{\mathbb{C}_{p}}$ such that $\tilde{x}^{(0)}=x$. Since we have $\pi_{\mathscr{K}} \otimes 1-1 \otimes\left[\tilde{\pi}_{\mathscr{K}}\right] \in I^{\prime}$, we have $\left(\pi_{\mathscr{K}} \otimes 1,1 \otimes\left[\tilde{\pi}_{\mathscr{K}}\right]\right) \subset$ $\left(\pi_{\mathscr{K}} \otimes 1, I^{\prime}\right)$. Note that if $x \in \mathcal{O}_{\mathscr{L}}$ is primitive, that is, $1, x, \ldots, x^{[\mathscr{L}: \mathscr{K}]-1}$ is an $\mathscr{O}_{\mathscr{K}}{ }^{-}$ basis of $O_{\mathscr{L}}$, then we have $I \subset\left(x \otimes 1-1 \otimes[\tilde{x}], I^{\prime}\right)$. Hence, we have only to prove the existence of a primitive element $x \in \mathscr{O}_{\mathscr{L}}$ satisfying $(x \otimes 1-1 \otimes[\tilde{x}])^{n} \in\left(\pi_{\mathscr{C}} \otimes 1, I^{\prime}\right)$ for some $n \in \mathbb{N}$. In the case $[\mathscr{L}: \mathscr{K}]=e_{\mathscr{L} / \mathscr{L}}, \pi_{\mathscr{L}}$ is a primitive element of $\mathrm{O}_{\mathscr{L}}$

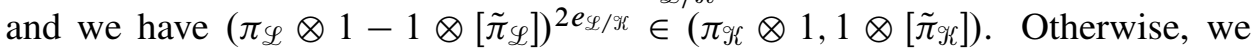
have $[\mathscr{L}: \mathscr{K}]=\left[k_{\mathscr{L}}: k_{\mathscr{K}}\right]_{\text {insep }}=p$. If we choose $x \in \mathrm{O}_{\mathscr{L}}$ whose image in $\mathrm{O}_{\mathscr{L}} / \pi_{\mathscr{K}} \mathrm{O}_{\mathscr{L}}$ does not belong to $k_{\mathscr{H}}$, then $x$ is primitive by Nakayama's lemma. Moreover, if we choose $a \in \mathrm{O}_{\mathscr{K}}$ such that $x^{p} \equiv a \bmod \pi_{\mathscr{C}} \mathcal{O}_{\mathscr{L}}$, then we have

$$
(x \otimes 1-1 \otimes[\tilde{x}])^{p} \equiv a \otimes 1-1 \otimes[\tilde{a}] \bmod \left(\pi_{\mathscr{K}} \otimes 1,1 \otimes\left[\tilde{\pi}_{\mathscr{K}}\right]\right)
$$

and $a \otimes 1-1 \otimes[\tilde{a}] \in I^{\prime}$, which implies the assertion.

(iii) We denote the map by $i$ and we will construct the inverse. By replacing $\mathscr{K}$ and $\mathscr{L}$ by $\mathscr{K}^{\mathrm{ur}}$ and $\mathscr{L}^{\mathrm{ur}}$, we may assume $\left[k_{\mathscr{L}}: k_{\mathscr{K}}\right]_{\mathrm{sep}}=1$. By (ii), we identify $\mathbb{A}_{\text {inf, } \mathbb{C}_{p} / \mathscr{L}}$ with $O_{\mathscr{L}} \otimes_{\mathcal{O}_{\mathscr{K}}} \mathbb{A}_{\text {inf, } \mathbb{C}_{p} / \mathscr{K}}$. Since $\mathscr{L} / \mathscr{K}$ is étale, by a similar argument as in the proof of (i), we have a unique $\mathscr{K}$-algebra homomorphism

$$
j: \mathscr{L} \rightarrow \mathbb{A}_{\text {inf, } \mathbb{C}_{p} / \mathscr{F}}\left[p^{-1}\right] /\left(\operatorname{ker} \theta_{\mathbb{C}_{p} / \mathscr{K}}\right)^{n}
$$

such that $\theta_{\mathbb{C}_{p} / \mathscr{K}}: \mathbb{A}_{\text {inf, } \mathbb{C}_{p} / \mathscr{K}}\left[p^{-1}\right] /\left(\operatorname{ker} \theta_{\mathbb{C}_{p} / \mathscr{K}}\right)^{n} \rightarrow \mathbb{C}_{p}$ is an $\mathscr{L}$-algebra homomor-

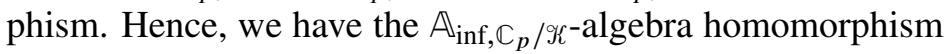

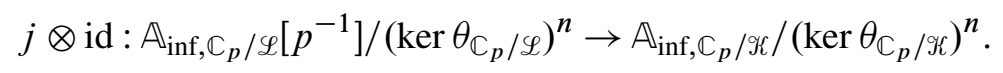

By construction, we have $(j \otimes \mathrm{id}) \circ i=\mathrm{id}$. To prove $i \circ(j \otimes \mathrm{id})=\mathrm{id}$, we have only to prove that $i \circ(j \otimes \mathrm{id})$ is an $\mathscr{L}$-algebra homomorphism, which follows from the uniqueness of $j$.

Remark 3.5. We may identify $\mathbb{A}_{\text {inf, } \mathbb{C}_{p} / \mathbb{Q}_{p}}$ with $W\left(R_{\mathbb{C}_{p}}\right)$ [Fontaine 1994a, 1.2.4(e)] and the kernel of $\theta_{\mathbb{C}_{p} / \mathbb{Q}_{p}}$ is principal by [Fontaine 1994a, 2.3.3]. Moreover, if $\mathscr{K}=\mathscr{K}_{0}$ and $k_{\mathscr{K}}$ is perfect, then the canonical map $\mathbb{A}_{\mathrm{inf}, \mathbb{C}_{p} / \mathbb{Q}_{p}} \rightarrow \mathbb{A}_{\mathrm{inf}, \mathbb{C}_{p} / \mathscr{K}_{K}}$ is an isomorphism [Fontaine 1994a, 1.2.4(e)]. Note that we have no canonical choice of an embedding $W\left(k_{K}^{\mathrm{alg}}\right)\left[p^{-1}\right] \rightarrow \mathbb{C}_{p}$ when $k_{K}$ is imperfect, since different

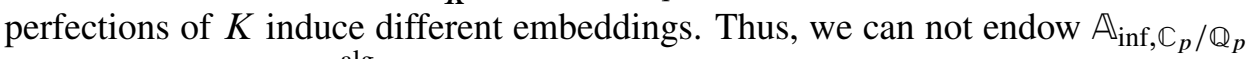
with a canonical $W\left(k_{K}^{\mathrm{alg}}\right)$-algebra structure induced by that of $\mathbb{A}_{\mathrm{inf}, \mathbb{C}_{p} / W\left(k_{K}^{\mathrm{alg}}\right)\left[p^{-1}\right]}$ via the above isomorphism as in the perfect residue field case. 
3B. $\mathbb{B}_{\mathbf{d R}}$ and $\mathbb{B}_{\mathbf{H T}}$. We define $\mathbb{B}_{\mathrm{dR}, \mathbb{C}_{p} / \mathscr{K}}^{+}:=\lim _{\leftarrow} \mathbb{A}_{\text {inf, } \mathbb{C}_{p} / \mathscr{K}}\left[p^{-1}\right] /\left(\operatorname{ker} \theta_{\mathbb{C}_{p} / \mathscr{K}}\right)^{n}$ and

$$
t:=\log ([\varepsilon])=\sum_{n \in \mathbb{N}_{>0}}(-1)^{n-1} \frac{([\varepsilon]-1)^{n}}{n} \in \mathbb{B}_{\mathrm{dR}, \mathbb{C}_{p} / \mathbb{Q}_{p}}^{+}
$$

with $\varepsilon:=\left(1, \zeta_{p}, \zeta_{p^{2}}, \ldots\right) \in R_{\mathbb{C}_{p}}$. We also define $\mathbb{B}_{\mathrm{dR}, \mathbb{C}_{p} / \mathscr{K}}:=\mathbb{B}_{\mathrm{dR}, \mathbb{C}_{p} / \mathscr{K}}^{+}\left[t^{-1}\right]$. We denote the projection $\mathbb{B}_{\mathrm{dR}, \mathbb{C}_{p} / \mathscr{K}}^{+} \rightarrow \mathbb{C}_{p}$ by $\theta_{\mathbb{C}_{p} / \mathscr{K}}$ again. Then, $\mathbb{B}_{\mathrm{dR}, \mathbb{C}_{p} / \mathscr{K}}^{+}$is a Hausdorff complete local ring with maximal ideal $\operatorname{ker} \theta_{\mathbb{C}_{p} / \mathscr{K}}$. Moreover, $\mathbb{B}_{\mathrm{dR}, \mathbb{C}_{p} / \mathscr{K}}$ is an integral domain. In fact, by the following explicit description of $\mathbb{B}_{\mathrm{dR}, \mathbb{C}_{p} / \mathscr{K}}$, it follows from the fact that $\mathbb{B}_{\mathrm{dR}, \mathbb{C}_{p} / \mathbb{Q}_{p}}$ is a field (Remark 3.6(ii) below).

We define the canonical topology on $\mathbb{B}_{\mathrm{dR}, \mathbb{C}_{p} / \mathscr{\kappa}}^{+}$as follows. We regard

$$
\mathbb{A}_{\text {inf, } \mathbb{C}_{p} / \mathscr{K}}\left[p^{-1}\right] /\left(\operatorname{ker} \theta_{\mathbb{C}_{p} / \mathscr{K}}\right)^{n}
$$

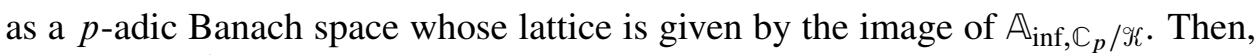
we endow $\mathbb{B}_{\mathrm{dR}, \mathbb{C}_{p} / \mathscr{K}}^{+}$with the inverse limit topology, which is a Fréchet complete $\mathscr{K}$-algebra. We also endow $\mathbb{B}_{\mathrm{dR}, \mathbb{C}_{p} / \mathscr{K}}$ with a limit of Fréchet topology by regarding $\mathbb{B}_{\mathrm{dR}, \mathbb{C}_{p} / \mathscr{C}}$ as the direct limit of $\mathbb{B}_{\mathrm{dR}, \mathbb{C}_{p} / \mathscr{K}}^{+}$with respect to the multiplication by $t^{-1}$. Let $v_{\mathrm{dR}, \mathbb{C}_{p} / \mathscr{K}}^{(n)}$ be the semivaluation of $\mathbb{B}_{\mathrm{dR}, \mathbb{C}_{p} / \mathscr{K}}^{+}$induced by the $p$-adic semivaluation of $\mathbb{B}_{\mathrm{dR}, \mathbb{C}_{p} / \mathscr{K}}^{+} /\left(\operatorname{ker} \theta_{\mathbb{C}_{p} / \mathscr{K}}\right)^{n}$ defined by the lattice

$$
\operatorname{Im}\left(\mathbb{A}_{\mathrm{inf}, \mathbb{C}_{p} / \mathscr{K}} \stackrel{\text { can. }}{\longrightarrow} \mathbb{B}_{\mathrm{dR}, \mathbb{C}_{p} / \mathscr{K}}^{+} /\left(\operatorname{ker} \theta_{\mathbb{C}_{p} / \mathscr{K}}\right)^{n}\right) .
$$

Obviously, the semivaluations $\left\{v_{\mathrm{dR}, \mathbb{C}_{p} / \mathscr{K}}^{(n)}\right\}_{n \in \mathbb{N}}$ are decreasing.

We will give an explicit description of $\mathbb{B}_{\mathrm{dR}, \mathbb{C}_{p} / \Re \text {. Let }}^{+}$ $\mathbb{B}_{\mathrm{dR}, \mathbb{C}_{p} / \mathbb{Q}_{p}}^{+}\left\{\mathbf{u}_{J_{\mathscr{K}}}\right\}:=$

$$
\left\{\sum_{\boldsymbol{n} \in \mathbb{N} \oplus J_{K}} a_{\boldsymbol{n}} \mathbf{u}^{\boldsymbol{n}} \mid a_{\boldsymbol{n}} \in \mathbb{B}_{\mathrm{dR}, \mathbb{C}_{p} / \mathbb{Q}_{p}}^{+},\left\{v_{\mathrm{dR}, \mathbb{C}_{p} / \mathbb{Q}_{p}}^{(r)}\left(a_{\boldsymbol{n}}\right)\right\}_{|\boldsymbol{n}|=n} \rightarrow \infty \text { for all } n, r \in \mathbb{N}\right\} .
$$

This is a $\mathbb{B}_{\mathrm{dR}, \mathbb{C}_{p} / \mathbb{Q}_{p}}^{+}$-algebra. Then, the canonical $\mathbb{B}_{\mathrm{dR}, \mathbb{C}_{p} / \mathbb{Q}_{p}}^{+}$-algebra homomorphism

$$
\iota_{\mathrm{dR}, \mathbb{C}_{p} / \mathscr{K}}: \mathbb{B}_{\mathrm{dR}, \mathbb{C}_{p} / \mathbb{Q}_{p}}^{+}\left\{\mathbf{u}_{J_{K}}\right\} \rightarrow \mathbb{B}_{\mathrm{dR}, \mathbb{C}_{p} / \mathscr{K}}^{+} ; \quad \mathbf{u}^{\boldsymbol{n}} \mapsto \boldsymbol{u}^{\boldsymbol{n}}
$$

is an isomorphism. To prove this, by Remark 3.6(ii) below, we may reduce to the case $\mathscr{K}=\mathscr{K}_{0}$. In this case, the assertion follows from the explicit description

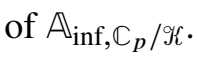

For $n \in \mathbb{N}$, let Fil ${ }^{n} \mathbb{B}_{\mathrm{dR}, \mathbb{C}_{p} / \mathscr{K}}^{+}$be the closed ideal of $\mathbb{B}_{\mathrm{dR}, \mathbb{C}_{p} / \mathscr{K}}^{+}$generated by the ideal $\left(\operatorname{ker} \theta_{\mathbb{C}_{p} / \mathscr{T}}\right)^{n}$. We endow $\mathbb{B}_{\mathrm{dR}, \mathbb{C}_{p} / \mathscr{K}}$ with the decreasing filtration defined by $\mathrm{Fil}^{n} \mathbb{B}_{\mathrm{dR}, \mathbb{C}_{p} / \mathscr{F}}:=\sum_{i+j=n} t^{i} \mathrm{Fil}^{j} \mathbb{B}_{\mathrm{dR}, \mathbb{C}_{p} / \mathscr{K}}^{+}$. Denote the graded $\mathbb{C}_{p}$-algebra associated to the filtration by $\mathbb{B}_{\mathrm{HT}, \mathbb{C}_{p} / \mathscr{\kappa}}$. We also denote by $v_{j}$ the image of $u_{j} / t$ in $\mathbb{B}_{\mathrm{HT}, \mathbb{C}_{p} / K, 0}$ for $j \in J_{K}$. Since the filtration is compatible with the multiplication by $t$, that is, $t^{m} \mathrm{Fil}^{n} \mathbb{B}_{\mathrm{dR}, \mathbb{C}_{p} / \mathscr{K}}=\mathrm{Fil}^{n+m} \mathbb{B}_{\mathrm{dR}, \mathbb{C}_{p} / \mathscr{K}}$, we have an isomorphism $\mathbb{B}_{\mathrm{HT}, \mathbb{C}_{p} / \mathscr{K}} \cong \bigoplus_{n \in \mathbb{Z}} \mathbb{B}_{\mathrm{HT}, \mathbb{C}_{p} / \mathscr{K}, 0} t^{n}$. 
For $n \in \mathbb{N}$, let

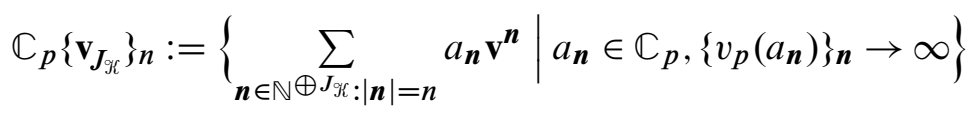

and $\mathbb{C}_{p}\left\{\mathbf{v}_{J_{\mathscr{H}}}\right\}:=\bigoplus_{n \in \mathbb{N}} \mathbb{C}_{p}\left\{\mathbf{v}_{J_{\mathscr{H}}}\right\}_{n}$. We have a $\mathbb{C}_{p}$-algebra homomorphism

$$
\iota_{\mathrm{HT}, \mathbb{C}_{p} / \mathscr{K}, 0}: \mathbb{C}_{p}\left\{\mathbf{v}_{J_{\mathscr{K}}}\right\} \rightarrow \mathbb{B}_{\mathrm{HT}, \mathbb{C}_{p} / \mathscr{K}, 0} ; \quad \mathbf{v}^{\boldsymbol{n}} \mapsto \boldsymbol{v}^{\boldsymbol{n}},
$$

which is an isomorphism. One reduces to the case $\mathscr{K}=\mathscr{K}_{0}$ by Remark 3.6(ii) below. Then, the assertion follows from the above explicit description of $\mathbb{B}_{\mathrm{dR}, \mathbb{C}_{p} / \mathscr{K}}^{+}$ and the formula of the semivaluation $v_{\mathrm{dR}, \mathbb{C}_{p} / \mathscr{K}}^{(n)}$ (Remark 3.6(iii) below). By this description, $\mathbb{B}_{\mathrm{HT}, \mathbb{C}_{p} / \mathscr{K}}$ is an integral domain.

Remark 3.6. (i) (The perfect residue field case) Assume that $k_{\mathscr{F}}$ is perfect. Then, we have a canonical isomorphism $\mathbb{B}_{\odot, \mathbb{C}_{p} / \mathbb{Q}_{p}} \rightarrow \mathbb{B}_{\odot, \mathbb{C}_{p} / \mathscr{F}}$ for $\odot \in\{\mathrm{dR}, \mathrm{HT}\}$. Moreover, $\mathbb{B}_{\mathrm{dR}, \mathbb{C}_{p} / \mathbb{Q}_{p}}$ is a complete discrete valuation field of equal characteristic 0 with valuation ring $\mathbb{B}_{\mathrm{dR}, \mathbb{C}_{p} / \mathbb{Q}_{p}}^{+}, t$ is a uniformizer and the residue field is $\mathbb{C}_{p}$. We also have an isomorphism $\mathbb{B}_{\mathrm{HT}, \mathbb{C}_{p} / \mathbb{Q}_{p}} \cong \bigoplus_{n \in \mathbb{Z}} \mathbb{C}_{p} t^{n}$. In fact, the first assertion follows from Remark 3.5 and the latter assertion reduces to the case where $k_{K}$ is perfect by regarding $\mathbb{C}_{p}$ as the $p$-adic completion of $\left(K^{\mathrm{pf}}\right)^{\mathrm{alg}}$ [Fontaine 1994a, 1.5.1].

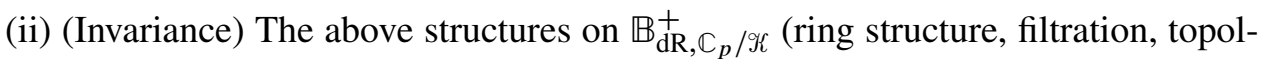
ogy) are invariant under finite or unramified extensions. As a consequence, we may regard $\mathbb{B}_{\mathrm{dR}, \mathbb{C}_{p} / \mathscr{K}}^{+}$as a $\mathscr{K}^{\mathrm{alg}}$-algebra and a similar invariance for $\mathbb{B}_{\mathrm{HT}, \mathbb{C}_{p} / \mathscr{K}}$ as a graded $\mathbb{C}_{p}$-algebra also holds. As for a filtered ring, the invariance follows from Lemma 3.4(iii). To prove the rest of the assertion, we have only to prove that for an unramified extension or a finite extension $\mathscr{L} / \mathscr{K}$, the $p$-adic semivaluations $v_{\mathrm{dR}, \mathbb{C}_{p} / \mathscr{K}}^{(n)}$ and $v_{\mathrm{dR}, \mathbb{C}_{p} / \mathscr{L}}^{(n)}$ are equivalent for all $n \in \mathbb{N}$. The unramified case follows from Lemma 3.4(i). In the other case, let $\Lambda_{\mathscr{K}}^{(n)}$ (resp. $\Lambda_{\mathscr{L}}^{(n)}$ ) be the image of $\mathbb{A}_{\mathrm{inf}, \mathbb{C}_{p} / \mathscr{K}}\left(\right.$ resp. $\left.\mathbb{A}_{\mathrm{inf}, \mathbb{C}_{p} / \mathscr{L}}\right)$ in $\mathbb{B}_{\mathrm{dR}, \mathbb{C}_{p} / \mathscr{K}}^{+} /\left(\operatorname{ker} \theta_{\mathbb{C}_{p} / \mathscr{K}}\right)^{n}$. Replacing $\mathscr{K}$ by the maximal unramified extension of $\mathscr{K}$ in $\mathscr{L}$, we may assume that $\mathscr{L} / \mathscr{K}$ satisfies the

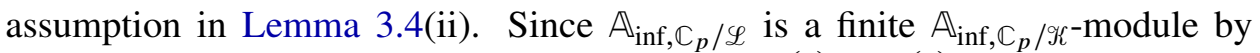
Lemma 3.4(ii), there exists $m \in \mathbb{N}$ such that $p^{m} \Lambda_{\mathscr{L}}^{(n)} \subset \Lambda_{\mathscr{K}}^{(n)}$ by Lemma 3.4(iii). Since we have $\Lambda_{\mathscr{K}}^{(n)} \subset \Lambda_{\mathscr{L}}^{(n)}$ by definition, the two $p$-adic topologies induced by the lattices $\Lambda_{\mathscr{K}}^{(n)}$ and $\Lambda_{\mathscr{L}}^{(n)}$ respectively are equivalent, which implies the assertion.

(iii) Assume $\mathscr{K}=\mathscr{K}_{0}$. Then, we have the formula

$$
v_{\mathrm{dR}, \mathbb{C}_{p} / \mathscr{K}}^{(n)}(x)=\inf _{|\boldsymbol{n}|<n} v_{\mathrm{dR}, \mathbb{C}_{p} / \mathbb{Q}_{p}}^{(n)}\left(a_{\boldsymbol{n}}\right),
$$

where we have $x=\sum_{\boldsymbol{n} \in \mathbb{N} \oplus J_{\mathscr{T}}} a_{\boldsymbol{n}} \boldsymbol{u}^{\boldsymbol{n}} \in \mathbb{B}_{\mathrm{dR}, \mathbb{C}_{p} / \mathscr{K}}^{+}$with $a_{\boldsymbol{n}} \in \mathbb{B}_{\mathrm{dR}, \mathbb{C}_{p} / \mathbb{Q}_{p}}^{+}$. This follows from the explicit description of $\mathbb{A}_{\text {inf, }, \mathbb{C}_{p} / \mathscr{F}}$. 
3C. Connections on $\mathbb{B}_{\mathbf{d R}}$ and $\mathbb{B}_{\mathbf{H T}}$. We denote by $\hat{\Omega}_{\mathscr{K}}^{q} \hat{\otimes}_{\mathscr{K}} \mathbb{B}_{\mathrm{dR}, \mathbb{C}_{p} / \mathscr{K}}$ the direct limit $\underset{\lim }{\longrightarrow} \hat{\Omega}_{\mathscr{K}}^{q} \hat{\otimes}_{\mathscr{K}} \mathbb{B}_{\mathrm{dR}, \mathbb{C}_{p} / \mathscr{K}}^{+}$, where the transition maps are the multiplication by $1 \otimes t^{-1}$. $\overrightarrow{T h e n}$, the canonical derivation $d: \mathscr{K} \rightarrow \widehat{\Omega}_{\mathscr{K}}^{1}$ uniquely extends to a $\mathbb{B}_{\mathrm{dR}, \mathbb{C}_{p} / \mathbb{Q}_{p}}$-linear continuous derivation

$$
\nabla: \mathbb{B}_{\mathrm{dR}, \mathbb{C}_{p} / \mathscr{K}} \rightarrow \hat{\Omega}_{\mathscr{K}}^{1} \hat{\otimes}_{\mathscr{K}} \mathbb{B}_{\mathrm{dR}, \mathbb{C}_{p} / \mathscr{K}}
$$

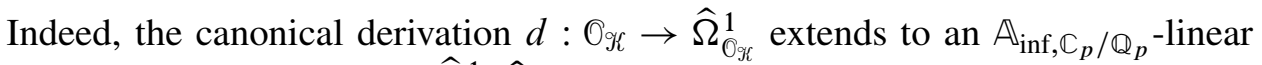
derivation $d: \mathbb{A}_{\mathrm{inf}, \mathbb{C}_{p} / \mathscr{K}} \rightarrow \widehat{\Omega}_{\mathscr{O}_{\mathscr{K}}}^{1} \hat{\otimes}_{\mathscr{O}_{\mathscr{K}}} \mathbb{A}_{\mathrm{inf}, \mathbb{C}_{p} / \mathscr{K}}$ by the construction of $\mathbb{A}_{\text {inf }}$. After inverting $p$, then taking the $\operatorname{ker} \theta_{\mathbb{C}_{p} / \mathscr{K}}$-adic Hausdorff completion, we obtain a desired derivation. Since the image of $\mathscr{K} \otimes_{\mathbb{Z}} \mathbb{B}_{\mathrm{dR}, \mathbb{C}_{p} / \mathbb{Q}_{p}}$ is dense in $\mathbb{B}_{\mathrm{dR}, \mathbb{C}_{p} / \mathscr{K}}$ by construction, the uniqueness follows. More precisely, if we denote by $\left\{\partial_{j}\right\}_{j \in J_{\gamma_{C}}}$ the derivations on $\mathbb{B}_{\mathrm{dR}, \mathbb{C}_{p} / \mathscr{K}}$ given by $\nabla(x)=\sum_{j \in J_{\mathscr{C}}} d t_{j} \otimes \partial_{j}(x)$, then $\left\{\partial_{j}\right\}_{j \in J_{\mathscr{K}}}$ are

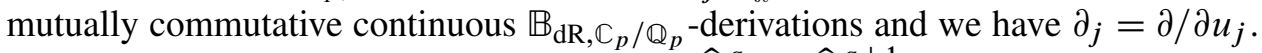
More generally, the exterior derivation $d_{q}: \widehat{\Omega}_{\mathscr{K}}^{q} \rightarrow \widehat{\Omega}_{\mathscr{K}}^{q+1}$ for $q \in \mathbb{N}_{>0}$ uniquely extends to a $\mathbb{B}_{\mathrm{dR}, \mathbb{C}_{p} / \mathbb{Q}_{p}}$-linear continuous homomorphism

$$
\nabla_{q}: \hat{\Omega}_{\mathscr{K}}^{q} \hat{\otimes}_{\mathscr{K}} \mathbb{B}_{\mathrm{dR}, \mathbb{C}_{p} / \mathscr{K}} \rightarrow \widehat{\Omega}_{\mathscr{K}}^{q+1} \hat{\otimes}_{\mathscr{K}} \mathbb{B}_{\mathrm{dR}, \mathbb{C}_{p} / \mathscr{K}}
$$

such that we have $\nabla_{q}(\omega \otimes x)=\nabla_{q}(\omega) \otimes x+(-1)^{q} \omega \wedge \nabla(x)$ for $x \in \mathbb{B}_{\mathrm{dR}, \mathbb{C}_{p} / \mathscr{K}}$ and $\omega \in \widehat{\Omega}_{\mathscr{K}}^{q}$. Obviously, the connection $\nabla$ satisfies Griffith transversality

$$
\nabla\left(\mathrm{Fil}^{n} \mathbb{B}_{\mathrm{dR}, \mathbb{C}_{p} / \mathscr{K}}\right) \subset \hat{\Omega}_{\mathscr{K}}^{1} \hat{\otimes}_{\mathscr{K}} \mathrm{Fil}^{n-1} \mathbb{B}_{\mathrm{dR}, \mathbb{C}_{p} / \mathscr{K}}
$$

for $n \in \mathbb{Z}$. These connections are invariant under finite or unramified extensions by Lemma 1.10(iii) and Remark 3.6(ii).

Notation. We will use the following notation:

$$
\begin{aligned}
& \mathbb{B}_{\mathrm{dR}, \mathbb{C}_{p} / \mathscr{K}}^{\nabla+}:=\left(\mathbb{B}_{\mathrm{dR}, \mathbb{C}_{p} / \mathscr{K}}^{+}\right)^{\nabla=0}, \mathbb{B}_{\mathrm{dR}, \mathbb{C}_{p} / \mathscr{K}}^{\nabla}:=\left(\mathbb{B}_{\mathrm{dR}, \mathbb{C}_{p} / \mathscr{K}}\right)^{\nabla=0}
\end{aligned}
$$

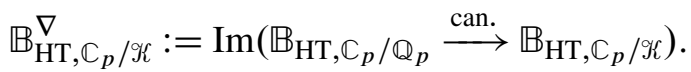

We endow the first two rings with induced filtrations and the last one with an induced graded structure. Note that these rings are invariant under finite or unramified extensions of $\mathscr{K}$ and that $\mathbb{B}_{\mathrm{dR}, \mathbb{C}_{p} / \mathscr{K}}^{\nabla+}$ and $\mathbb{B}_{\mathrm{dR}, \mathbb{C}_{p} / \mathscr{K}}^{\nabla}$ (resp. $\mathbb{B}_{\mathrm{HT}, \mathbb{C}_{p} / \mathscr{K}}^{\nabla}$ ) have a canonical $\left(\mathscr{K}_{\text {can }}\right)^{\text {alg }}$-algebra (resp. $\mathbb{C}_{p}$-algebra) structure. By the above description of the connection and the explicit descriptions of $\mathbb{B}_{\mathrm{dR}, \mathbb{C}_{p} / \mathscr{K}}$ and $\mathbb{B}_{\mathrm{HT}, \mathbb{C}_{p} / \mathscr{K}}$, we have:

Lemma 3.7. The canonical maps

$$
\mathbb{B}_{\mathrm{dR}, \mathbb{C}_{p} / \mathbb{Q}_{p}}^{+} \rightarrow \mathbb{B}_{\mathrm{dR}, \mathbb{C}_{p} / \mathscr{\kappa}}^{\nabla+}, \quad \mathbb{B}_{\mathrm{dR}, \mathbb{C}_{p} / \mathbb{Q}_{p}} \rightarrow \mathbb{B}_{\mathrm{dR}, \mathbb{C}_{p} / \mathscr{\kappa}}^{\nabla}, \quad \mathbb{B}_{\mathrm{HT}, \mathbb{C}_{p} / \mathbb{Q}_{p}} \rightarrow \mathbb{B}_{\mathrm{HT}, \mathbb{C}_{p} / \mathscr{K}}^{\nabla}
$$

are isomorphisms. These maps are compatible with filtrations and gradings. 
Remark 3.8. Assume that $\left[k_{\mathscr{K}}: k_{\mathscr{K}}^{p}\right]<\infty$. Since $\widehat{\Omega}_{\mathscr{K}}^{1}$ is a finite-dimensional $\mathscr{K}$ vector space (Remark 1.11), the connection $\nabla: \mathbb{B}_{\mathrm{dR}, \mathbb{C}_{p} / \mathscr{K}} \rightarrow \widehat{\Omega}_{\mathscr{K}}^{1} \otimes_{\mathscr{K}} \mathbb{B}_{\mathrm{dR}, \mathbb{C}_{p} / \mathscr{K}}$ induces a $\mathbb{B}_{\mathrm{HT}, \mathbb{C}_{p} / \mathbb{Q}_{p}}$-linear derivation

$$
\nabla: \mathbb{B}_{\mathrm{HT}, \mathbb{C}_{p} / \mathscr{K}} \rightarrow \hat{\Omega}_{\mathscr{K}}^{1} \otimes_{\mathscr{K}} \mathbb{B}_{\mathrm{HT}, \mathbb{C}_{p} / \mathscr{K}} .
$$

More precisely, if we denote by $\left\{\partial_{j}\right\}_{j \in J_{\mathscr{K}}}$ the derivations on $\mathbb{B}_{\mathrm{HT}, \mathbb{C}_{p} / \mathscr{K}}$ defined as above, then, by the explicit description of $\mathbb{B}_{\mathrm{HT}, \mathbb{C}_{p} / \mathscr{K}},\left\{\partial_{j}\right\}_{j \in J_{\mathscr{K}}}$ are commuting $\mathbb{B}_{\mathrm{HT}, \mathbb{C}_{p} / \mathbb{Q}_{p}}$-linear derivations and we have $\partial_{j}=t \partial / \partial v_{j}$. In particular, $\mathbb{B}_{\mathrm{HT}, \mathbb{C}_{p} / \mathscr{K}}^{\nabla}$ coincides with $\left(\mathbb{B}_{\mathrm{HT}, \mathbb{C}_{p} / \mathscr{K}}\right)^{\nabla=0}$. In the general case, we must handle complicated topologies to define such a connection. To avoid it, we define $\mathbb{B}_{\mathrm{HT}, \mathbb{C}_{p} / \mathscr{K}}^{\nabla}$ in an ad-hoc way as above.

We also have an analogue of Poincaré lemma.

Lemma 3.9. The complex

$$
0 \longrightarrow \mathbb{B}_{\mathrm{dR}, \mathbb{C}_{p} / \mathscr{K}}^{\nabla+} \stackrel{\text { inc. }}{\longrightarrow} \mathbb{B}_{\mathrm{dR}, \mathbb{C}_{p} / \mathscr{K}}^{+} \stackrel{\nabla}{\longrightarrow} \hat{\Omega}_{\mathscr{K}}^{1} \hat{\otimes}_{\mathscr{K}} \mathbb{B}_{\mathrm{dR}, \mathbb{C}_{p} / \mathscr{K}}^{+} \stackrel{\nabla_{1}}{\longrightarrow} \hat{\Omega}_{\mathscr{K}}^{2} \hat{\otimes}_{\mathscr{K}} \mathbb{B}_{\mathrm{dR}, \mathbb{C}_{p} / \mathscr{K}}^{+}
$$

is exact.

Proof. By the invariance of the above complex under a finite extension, we may assume $\mathscr{K}=\mathscr{K}_{0}$. Recall the explicit description of $\mathbb{B}_{\mathrm{dR}, \mathbb{C}_{p} / \mathscr{K}}^{+}$in Section 3B. Since we have $v_{p}(\boldsymbol{n} !) \leq|\boldsymbol{n}|$ for $\boldsymbol{n} \in \mathbb{N} \oplus J_{\mathscr{T}}, x \in \mathbb{B}_{\mathrm{dR}, \mathbb{C}_{p} / \mathscr{K}}^{+}$is written uniquely in the form $x=\sum_{\boldsymbol{n} \in \mathbb{N} \oplus J_{\Re \ell}} a_{\boldsymbol{n}} \boldsymbol{u}^{[\boldsymbol{n}]}$ with $a_{\boldsymbol{n}} \in \mathbb{B}_{\mathrm{dR}, \mathbb{C}_{p} / \mathbb{Q}_{p}}^{+}$such that $\left\{v_{\mathrm{dR}, \mathbb{C}_{p} / \mathbb{Q}_{p}}^{(r)}\left(a_{\boldsymbol{n}}\right)\right\}_{|\boldsymbol{n}|=n} \rightarrow \infty$ for all $r, n \in \mathbb{N}$. Moreover, we have the inequality

$$
\inf _{|\boldsymbol{n}|<r} v_{\mathrm{dR}, \mathbb{C}_{p} / \mathbb{Q}_{p}}^{(r)}\left(a_{\boldsymbol{n}}\right)+r>\inf _{|\boldsymbol{n}|<r} v_{\mathrm{dR}, \mathbb{C}_{p} / \mathbb{Q}_{p}}^{(r)}\left(\boldsymbol{n} ! a_{\boldsymbol{n}}\right)=v_{\mathrm{dR}, \mathbb{C}_{p} / \mathscr{K}}^{(r)}(x)
$$

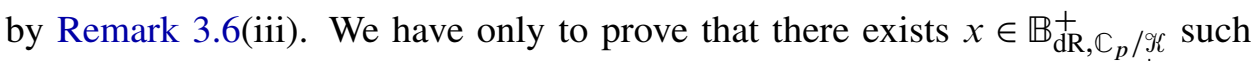
that $\nabla(x)=\omega$ for $\omega \in \operatorname{ker} \nabla_{1}$. Write $\omega=\sum_{j \in J_{\mathscr{K}}} d t_{j} \otimes \lambda_{j}$ with $\lambda_{j} \in \mathbb{B}_{\mathrm{dR}, \mathbb{C}_{p} / \mathscr{K}}^{+}$ such that $\left\{v_{\mathrm{dR}, \mathbb{C}_{p} / \mathscr{F}}^{(r)}\left(\lambda_{j}\right)\right\}_{j \in J_{\mathscr{}}} \rightarrow \infty$ for all $r \in \mathbb{N}$. The assumption $\omega \in \operatorname{ker} \nabla_{1}$ implies that we have $\partial_{j^{\prime}}\left(\lambda_{j}\right)=\partial_{j}\left(\lambda_{j^{\prime}}\right)$ for $j, j^{\prime} \in J_{\mathscr{K}}$. As above, we can write $\lambda_{j}=\sum_{\boldsymbol{n} \in \mathbb{N} \oplus J_{\mathscr{K}}} \lambda_{j, \boldsymbol{n}} \boldsymbol{u}^{[\boldsymbol{n}]}$, where $\lambda_{j, \boldsymbol{n}} \in \mathbb{B}_{\mathrm{dR}, \mathbb{C}_{p} / \mathbb{Q}_{p}}^{+}$satisfies the convergence condi-

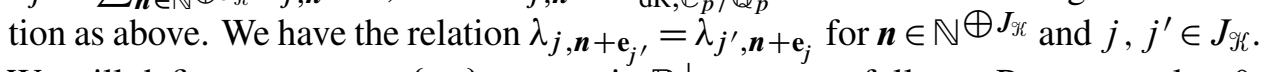
We will define a sequence $\left\{a_{\boldsymbol{n}}\right\}_{\boldsymbol{n} \in \mathbb{N} \oplus J_{Y C}}$ in $\mathbb{B}_{\mathrm{dR}, \mathbb{C}_{p} / \mathbb{Q}_{p}}^{+}$as follows: Put $a_{\mathbf{0}}$ equal to 0 . For $\boldsymbol{n} \neq \mathbf{0}$, choose any $j \in J_{\mathscr{K}}$ such that $n_{j} \neq 0$ and define $a_{\boldsymbol{n}}:=\lambda_{j, \boldsymbol{n}-\mathbf{e}_{j}}$. By the above relation, this is independent of the choice of $j$. To prove the assertion, it suffices to prove that we have $\left\{v_{\mathrm{dR}, \mathbb{C}_{p} / \mathbb{Q}_{p}}^{(r)}\left(a_{\boldsymbol{n}}\right)\right\}_{|\boldsymbol{n}|=n} \rightarrow \infty$ for all $r, n \in \mathbb{N}$. Indeed, if this is proved, we see that the element $x:=\sum_{\boldsymbol{n} \in \mathbb{N} \oplus J_{\mathscr{K}}} a_{\boldsymbol{n}} \mathbf{u}^{[\boldsymbol{n}]}$ belongs to $\mathbb{B}_{\mathrm{dR}, \mathbb{C}_{p} / \mathscr{K}}^{+}$ and we have $\nabla(x)=\omega$. We have only to prove that, for fixed $r, n, N \in \mathbb{N}$, we have $v_{\mathrm{dR}, \mathbb{C}_{p} / \mathbb{Q}_{p}}^{(r)}\left(a_{\boldsymbol{n}}\right) \geq N$ for all but finitely many $\boldsymbol{n} \in \mathbb{N} \oplus J_{\mathscr{F}}$ such that $|\boldsymbol{n}|=n$. We may assume $r \geq n$. Choose a finite subset $J$ of $J_{\mathscr{K}}$ such that $v_{\mathrm{dR}, \mathbb{C}_{p} / \mathscr{K}_{\mathcal{K}}}^{(r)}\left(\lambda_{j}\right) \geq r+N$ 
for $j \in J_{\mathscr{K}} \backslash J$. Let $\boldsymbol{n} \in \mathbb{N} \oplus J_{K}$ such that $|\boldsymbol{n}|=n$. If there exists $j \in J_{\mathscr{K}} \backslash J$ such that $n_{j} \neq 0$, then we have

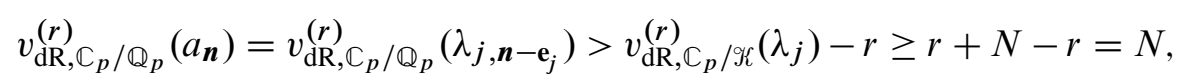

where the first inequality follows from inequality (1). This implies the assertion since our exceptional set $\left\{\boldsymbol{n} \in \mathbb{N}^{J}|| \boldsymbol{n} \mid=n\right\}$ is finite.

\section{D. Universal PD-thickenings.}

Defintion 3.10. A $p$-adically formal $\mathscr{O}_{\mathscr{K}}$-PD-thickening of $\mathbb{O}_{\mathbb{C}_{p}}$ is a triple

$$
\left(D, \theta_{D}, \gamma_{D}\right)
$$

where

- $D$ is a $p$-adically Hausdorff complete $0_{\mathscr{K}}$-algebra,

- $\theta_{D}: D \rightarrow \mathbb{O}_{\mathbb{C}_{p}}$ is a surjective $\mathscr{O}_{\mathscr{K}}$-algebra homomorphism,

- $\gamma_{D}$ is a PD-structure on $\operatorname{ker} \theta_{D}$, compatible with the canonical PD-structure on the ideal $(p)$.

Obviously, $p$-adically formal $\mathscr{O}_{\mathscr{K}}$-thickenings of $\mathbb{O}_{\mathbb{C}_{p}}$ form a category.

Theorem 3.11 [Fontaine 1994b, Théorème 2.2.1]. The category of p-adically formal $\mathbb{O}_{\mathscr{K}}$-thickenings of $\mathbb{O}_{\mathbb{C}_{p}}$ admits a universal object, that is, an initial object.

Such an object is unique up to a canonical isomorphism and we denote it by $\left(\mathbb{A}_{\text {cris, } \mathbb{C}_{p} / \mathscr{K}}, \theta_{\mathbb{C}_{p} / \mathscr{K}}, \gamma\right)$. Let's recall the construction. Let $\left(\mathscr{O}_{\mathscr{K}} \otimes_{\mathbb{Z}} W\left(R_{\mathbb{C}_{p}}\right)\right)^{\mathrm{PD}}$ be the PD-envelope of $\mathrm{O}_{\mathscr{K}} \otimes_{\mathbb{Z}} W\left(R_{\mathbb{C}_{p}}\right)$ with respect to the ideal

$$
\operatorname{ker}\left(\theta_{\mathbb{C}_{p} / \mathscr{K}}: \mathscr{O}_{\mathscr{K}} \otimes_{\mathbb{Z}} W\left(R_{\mathbb{C}_{p}}\right) \rightarrow \mathbb{O}_{\mathbb{C}_{p}}\right),
$$

compatible with the canonical PD-structure on the ideal $(p)$. Then, $\mathbb{A}_{\text {cris, } \mathbb{C}_{p} / \mathscr{K}}$ is the $p$-adic Hausdorff completion of $\left(\mathscr{O}_{\mathscr{K}} \otimes_{\mathbb{Z}} W\left(R_{\mathbb{C}_{p}}\right)\right)^{\mathrm{PD}}$.

Remark 3.12. (i) By [Fontaine 1994a, Remarques 2.2.3], if we have $\mathscr{K}=\mathscr{K}_{0}$ and $k_{\mathscr{K}}$ is perfect, then the canonical map $\mathbb{A}_{\text {cris, } \mathbb{C}_{p} / \mathbb{Q}_{p}} \rightarrow \mathbb{A}_{\text {cris, } \mathbb{C}_{p} / \mathscr{K}}$ is an isomorphism.

(ii) By a similar proof as Lemma 3.4(i), the canonical map

$$
\mathbb{A}_{\text {cris }, \mathbb{C}_{p} / \mathscr{K}} \rightarrow \mathbb{A}_{\text {cris }, \mathbb{C}_{p} / \mathscr{F} \text { ur }}
$$

is an isomorphism. In general, we have no invariance for $\mathbb{A}_{\text {cris, }, \mathbb{C}_{p} / \mathscr{F}}$ as in Remark 3.6(ii) even after inverting $p$.

If $\mathscr{K}=\mathscr{K}_{0}$ and $k_{\mathscr{K}}$ is perfect, then we have an explicit description of $\mathbb{A}_{\text {cris, } \mathbb{C}_{p} / \mathscr{K}}$ :

$$
\mathbb{A}_{\text {cris }, \mathbb{C}_{p} / \mathscr{K}}=\left\{\sum_{n \in \mathbb{N}} a_{n} \frac{\omega^{n}}{n !} \mid a_{n} \in \mathbb{A}_{\mathrm{inf}, \mathbb{C}_{p} / \mathscr{K}},\left\{v_{\mathrm{inf}, \mathbb{C}_{p} / \mathscr{K}}\left(a_{n}\right)\right\}_{n \in \mathbb{N}} \rightarrow \infty\right\},
$$


where $\omega$ denotes a generator of $\operatorname{ker}\left(\theta_{\mathbb{C}_{p} / \mathscr{K}}: \mathbb{A}_{\text {inf, } \mathbb{C}_{p} / \mathscr{K}} \rightarrow \mathbb{O}_{\mathbb{C}_{p}}\right)$. Note that the sequence $\left\{a_{n}\right\}_{n \in \mathbb{N}}$ is not uniquely determined. Moreover, we have $t \in \mathbb{A}_{\text {cris, }} \mathbb{C}_{p} / \mathscr{F}$ and $\mathbb{A}_{\text {cris, }} \mathbb{C}_{p} / \mathscr{K}$ is an integral domain of characteristic 0 whose PD-structure is given by $\gamma_{n}(x)=x^{[n]}=x^{n} / n$ ! for $x \in \operatorname{ker} \theta_{\mathbb{C}_{p} / \mathscr{F}}$. In fact, the assertions follow from the case $\mathscr{K}=K_{0}^{\mathrm{pf}}$ by Remark 3.5 and Remark 3.12(i), and the assertion in this case follows from [Fontaine 1994a, 2.3.3].

We define $\mathbb{B}_{\text {cris }, \mathbb{C}_{p} / \mathscr{K}}^{+}:=\mathbb{A}_{\text {cris, }, \mathbb{C}_{p} / \mathscr{K}}\left[p^{-1}\right]$ and $\mathbb{B}_{\text {cris, }, \mathbb{C}_{p} / \mathscr{K}}:=\mathbb{B}_{\text {cris, } \mathbb{C}_{p} / \mathscr{K}}^{+}\left[t^{-1}\right]$. We also define $\mathbb{A}_{s t, \mathbb{C}_{p} / \mathscr{K}}:=\mathbb{A}_{\text {cris, }, \mathbb{C}_{p} / \mathscr{K}}[\mathrm{x}]$, where $\mathrm{x}$ is a formal variable, and we set $\mathbb{B}_{\mathrm{st}, \mathbb{C}_{p} / \mathscr{K}}^{+}:=\mathbb{A}_{\mathrm{st}, \mathbb{C}_{p} / \mathscr{K}}\left[p^{-1}\right]$ and $\mathbb{B}_{\mathrm{st}, \mathbb{C}_{p} / \mathscr{K}}:=\mathbb{B}_{\mathrm{st}, \mathbb{C}_{p} / \mathscr{K}[}^{+}\left[t^{-1}\right]$. We define a monodromy

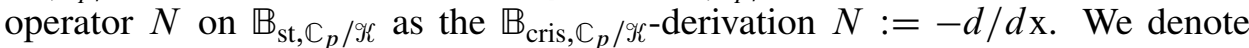
by $v_{\text {cris }, \mathbb{C}_{p} / \mathscr{K}}$ the $p$-adic semivaluation on $\mathbb{B}_{\text {cris }, \mathbb{C}_{p} / \mathscr{K}}^{+}\left(\right.$or $\mathbb{A}_{\text {cris }, \mathbb{C}_{p} / \mathscr{K}}$ ) defined by the lattice $\mathbb{A}_{\text {cris, }} \mathbb{C}_{p} / \mathscr{K}$.

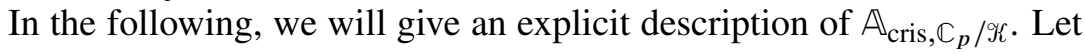

$$
\mathbb{A}_{\text {cris, } \mathbb{C}_{p} / \mathbb{Q}_{p}}\left\langle\mathbf{u}_{J_{\mathscr{C}}}\right\rangle
$$

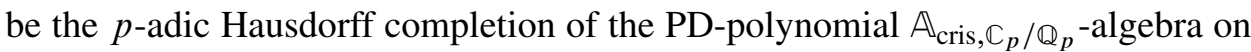
the indeterminates $\left\{\mathrm{u}_{j}\right\}_{j \in J_{\mathscr{K}}}$. Note that the PD-structure is given by $\gamma_{n}\left(\mathrm{u}_{j}\right)=$ $\mathrm{u}_{j}^{n} / n !=\mathrm{u}_{j}^{[n]}$ for $n \in \mathbb{N}$ and $j \in J_{\mathscr{K}}$. We also have

$$
\begin{aligned}
& \mathbb{A}_{\text {cris, } \mathbb{C}_{p} / \mathbb{Q}_{p}}\left\langle\mathbf{u}_{J_{\mathscr{S}}}\right\rangle=
\end{aligned}
$$

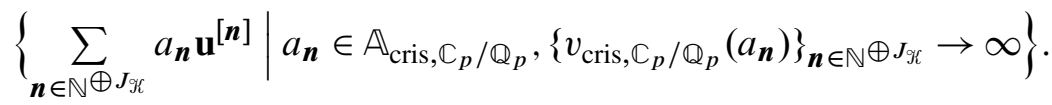

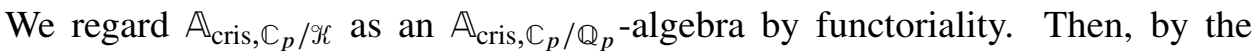

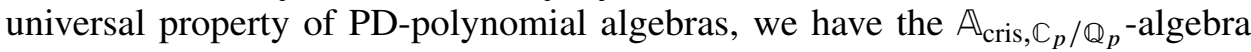
homomorphism

$$
\iota_{\text {cris, }, \mathbb{C}_{p} / \mathscr{K}}: \mathbb{A}_{\text {cris, }, \mathbb{C}_{p} / \mathbb{Q}_{p}}\left\langle\mathbf{u}_{J_{K}}\right\rangle \rightarrow \mathbb{A}_{\text {cris, }, \mathbb{C}_{p} / \mathscr{K}} ; \quad \mathbf{u}^{[n]} \mapsto \boldsymbol{u}^{[n]} .
$$

Lemma 3.13. If $\mathscr{K}=\mathscr{K}_{0}$, then $\iota_{\text {cris, } \mathbb{C}_{p} / \mathscr{K}}$ is an isomorphism. Moreover, we have

$$
v_{\text {cris }, \mathbb{C}_{p} / \mathscr{K}}(x)=\inf _{\boldsymbol{n} \in \mathbb{N} \oplus J_{\mathscr{K}}} v_{\text {cris, }, \mathbb{C}_{p} / \mathbb{Q}_{p}}\left(a_{\boldsymbol{n}}\right)
$$

for $x=\sum_{\boldsymbol{n} \in \mathbb{N} \oplus J_{\mathscr{H}}} a_{\boldsymbol{n}} \boldsymbol{u}^{[\boldsymbol{n}]} \in \mathbb{B}_{\text {cris, } \mathbb{C}_{p} / \mathscr{F}}^{+}$with $a_{\boldsymbol{n}} \in \mathbb{B}_{\text {cris, } \mathbb{C}_{p} / \mathbb{Q}_{p}}^{+}$.

We use the following lemma in the proof:

Lemma 3.14. We also assume that $\mathscr{K}=\mathscr{K}_{0}$ and we use the notation in Section $1 A$.

(i) If $R$ is a p-adically Hausdorff complete $\mathbb{Z}\left[T_{j}\right]_{j \in J_{\%}}$-algebra, then the canonical map

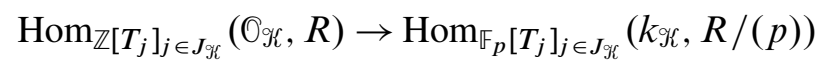


is bijective, where the $\mathbb{F}_{p}\left[T_{j}\right]_{j \in J_{\mathscr{F}}}$-algebra structure on $k_{\mathscr{K}}$ (resp. $\left.R /(p)\right)$ is given by $T_{j} \mapsto \bar{t}_{j}$ (resp. is induced by $\mathbb{Z}\left[T_{j}\right]_{j \in J_{\Im C}} \rightarrow R$ ). Moreover, the restriction map

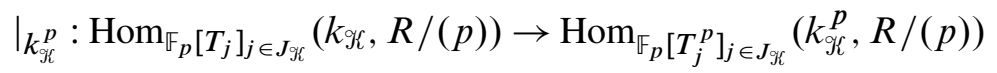

is bijective, where the $\mathbb{F}_{p}\left[T_{j}^{p}\right]_{j \in J_{\mathscr{K}}}$-algebra structure on $k_{\mathscr{K}}^{p}($ resp. $R /(p))$ is given by $T_{j}^{p} \mapsto t_{j}^{p}$ (resp. the composition of the inclusion $\mathbb{E}_{p}\left[T_{j}^{p}\right]_{j \in J_{\mathscr{S}}} \rightarrow \mathbb{F}_{p}\left[T_{j}\right]_{j \in J_{\mathscr{}}}$ and the above structure map $\left.\mathbb{F}_{p}\left[T_{j}\right]_{j \in J_{\mathscr{K}}} \rightarrow R /(p)\right)$.

(ii) Let $\vartheta: S \rightarrow R$ be a surjective homomorphism of p-adically Hausdorff complete $\mathbb{Z}\left[T_{j}\right]_{j \in J_{\mathscr{K}}}$-algebras, whose kernel admits a PD-structure, compatible with the canonical PD-structure on the ideal ( $p$ ). Then, the canonical map

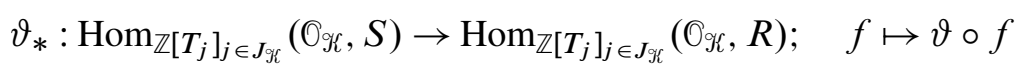

is bijective.

Proof. (i) The first claim follows from the $p$-adic formal étaleness of $\mathcal{O}_{\mathscr{K}} / \mathbb{Z}\left[T_{j}\right]_{j \in J_{\mathscr{K}}}$. The latter assertion follows by using the isomorphism of $k_{\mathscr{K}}^{p}$-algebras

$$
k_{\mathscr{K}}^{p}\left[T_{j}\right]_{j \in J_{\mathscr{K}}} /\left(\left\{T_{j}^{p}-\bar{t}_{j}^{p}\right\}_{j \in J_{\mathscr{K}}}\right) \cong k_{\mathscr{K}} ; \quad \bar{T}_{j} \mapsto \bar{t}_{j} .
$$

(ii) We denote by $\vartheta_{1}: S /(p) \rightarrow R /(p)$ the ring homomorphism induced by $\vartheta$. By the first assertion of (i), we have only to prove that the canonical map

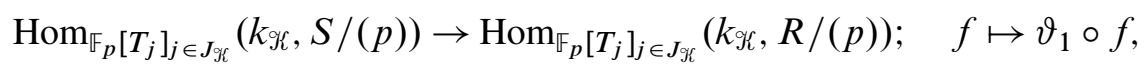

which is denoted by $\vartheta_{*}$ again, is bijective.

We first note the following: We regard $R /(p)$ as a quotient of $S /(p)$ by $\vartheta_{1}$. Let $x \in R /(p)$ and let $\hat{x}_{1}, \hat{x}_{2} \in S /(p)$ be lifts of $x$. Then, we have $\hat{x}_{1}-\hat{x}_{2} \in \operatorname{ker} \vartheta_{1}$. Since $a^{p}=p ! \gamma_{p}(a) \in p S$ for $a \in \operatorname{ker} \vartheta$, where $\gamma$ denotes a PD-structure on ker $\vartheta$, we have $\hat{x}_{1}^{p}=\hat{x}_{2}^{p}$. In particular, if we denote by $\hat{x} \in S /(p)$ a lift of $x \in R /(p)$, then $\hat{x}^{p}$ depends only on $x$.

We prove the injectivity. Let $\bar{f}: k_{\mathscr{K}} \rightarrow R /(p)$ be an $\mathbb{F}_{p}\left[T_{j}\right]_{j \in J_{\mathscr{K}}}$-algebra homomorphism and $f, f^{\prime}: k_{\mathscr{K}} \rightarrow S /(p)$ lifts of $\bar{f}$, that is, $\vartheta_{*}(f)=\vartheta_{*}\left(f^{\prime}\right)=\bar{f}$. For $\bar{x} \in k_{K}, f(\bar{x})$ and $f^{\prime}(\bar{x}) \in S /(p)$ are lifts of $\bar{f}(\bar{x}) \in R /(p)$, hence we have $f\left(\bar{x}^{p}\right)=f(\bar{x})^{p}=f^{\prime}(\bar{x})^{p}=f^{\prime}\left(\bar{x}^{p}\right)$ by the above remark. Hence, we

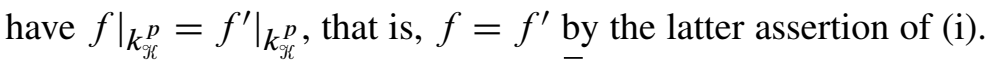

We prove the surjectivity. Let $\bar{f}: k_{\mathscr{K}} \rightarrow R /(p)$ be an $\mathbb{F}_{p}\left[T_{j}\right]_{j \in J_{\mathscr{K}}}$-algebra homomorphism. We have only to construct an $\mathbb{F}_{p}\left[T_{j}^{p}\right]_{j \in J_{\mathscr{K}}}$-algebra homomorphism $f: k_{\mathscr{F}}^{p} \rightarrow S /(p)$ such that $\left.\vartheta_{*}(f)\right|_{k_{\mathscr{C}}^{p}}$ coincides with $\left.\bar{f}\right|_{k_{\mathscr{C}}^{p}}$, where we endow $k_{\mathscr{K}}^{p}$ and $S /(p)$ with $\mathbb{E}_{p}\left[T_{j}^{p}\right]_{j \in J_{\mathscr{K}}}$-algebra structures by a similar way as in the statement of (i). In fact, we can uniquely extend $f$ to a $\mathbb{Z}\left[T_{j}\right]_{j \in J_{\mathscr{F}}}$-algebra homomorphism $f: k_{\mathscr{K}} \rightarrow S /(p)$ by the latter assertion of (i). Moreover, $\left.\left(\vartheta_{*}(f)\right)\right|_{k_{\digamma_{k}}^{p}}=$ $\vartheta_{*}\left(\left.f\right|_{k_{\Re}^{p}}\right)$ coincides with $\left.\bar{f}\right|_{k_{\Re}^{p}}$, which implies $\vartheta_{*}(f)=\bar{f}$ by the latter assertion 
of (i) again. The set-theoretic map $f: k_{\mathscr{q}}^{p} \rightarrow S /(p)$ taking $\bar{y}$ to $\hat{x}^{p}$, where $\hat{x} \in S /(p)$ is any lift of $\bar{f}\left(\bar{y}^{p^{-1}}\right) \in R /(p)$, is well-defined by the above remark. Moreover, $f$ is a $\mathbb{Z}\left[T_{j}\right]_{j \in J_{\mathscr{K}}}$-algebra homomorphism by a simple calculation and $\left.\vartheta_{*}(f)\right|_{k_{\mathscr{H}}^{p}}$ coincides with $\left.\bar{f}\right|_{k_{\mathscr{K}}^{p}}$ by construction, which implies the assertion.

Proof of Lemma 3.13. Obviously, we have only to prove the first assertion. Put $\mathscr{A}=$

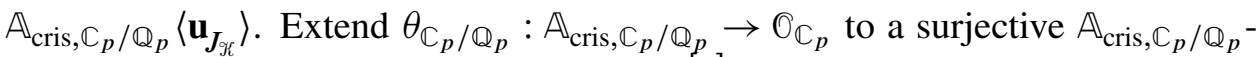
algebra homomorphism $\vartheta: \mathscr{A} \rightarrow \mathscr{O}_{\mathbb{C}_{p}}$ by $\vartheta\left(\mathbf{u}^{[\boldsymbol{n}]}\right)=0$. We first prove that $\mathscr{A}$ has an Oभi-algebra structure such that $\vartheta$ is an $O_{\mathscr{K}}$-algebra homomorphism.

Denote by $\omega$ a generator of the kernel of $\theta_{\mathbb{C}_{p} / \mathbb{Q}_{p}}: \mathbb{A}_{\text {cris, } \mathbb{C}_{p} / \mathbb{Q}_{p}} \rightarrow \mathbb{O}_{\mathbb{C}_{p}}$. Then, the PD-structure on the ideal $\operatorname{ker} \theta_{\mathbb{C}_{p} / \mathbb{Q}_{p}}$ of $\mathbb{A}_{\text {cris, }, \mathbb{C}_{p} / \mathbb{Q}_{p}}$ canonically extends to a PD-structure $\delta_{1}$ on the ideal $(\omega)$ of $\mathscr{A}$, compatible with the canonical PD-structure on the ideal $(p)$. By construction, the kernel of the map $\xi: \mathscr{A} \rightarrow \mathbb{A}_{\text {cris, } \mathbb{C}_{p} / \mathbb{Q}_{p}}$ taking $\mathbf{u}^{[n]}$ to 0 is endowed with a PD-structure $\delta_{2}$, compatible with the canonical PD-structure on the ideal $(p)$. Since $\mathscr{A}$ is an integral domain of characteristic 0 , $\delta_{1}$ and $\delta_{2}$ induce the same PD-structure on $(\omega) \cap \operatorname{ker} \xi$. Hence, by [Berthelot and Ogus 1978, Proposition 3.12], the ideal $\operatorname{ker} \vartheta=(\omega)+\operatorname{ker} \xi$ admits a PD-structure, compatible with the canonical PD-structure on the ideal $(p)$. Then, the assertion follows by applying Lemma 3.14(ii) to $\vartheta$ :

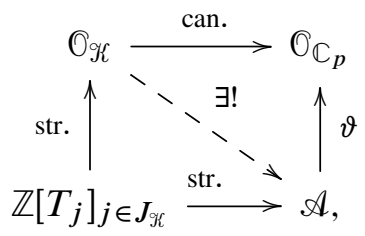

where the horizontal structure map is given by $T_{j} \mapsto \mathrm{u}_{j}+\left[\tilde{t}_{j}\right] \in \mathscr{A}$.

By the above $O_{\mathscr{K}}$-structure, we may regard $\mathscr{A}$ as a $p$-adically formal $\mathscr{O}_{\mathscr{K}}$-PD-

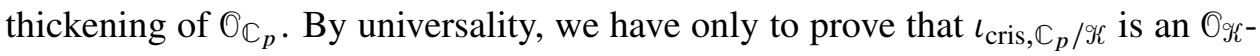
algebra homomorphism. Let $\alpha: \mathscr{O}_{\mathscr{K}} \rightarrow \mathbb{A}_{\text {cris, } \mathbb{C}_{p} / \mathscr{K}}$ be the composition of the structure map $\mathscr{O}_{\mathscr{K}} \rightarrow \mathscr{A}$ and $\iota_{\text {cris }, \mathbb{C}_{p} / \mathscr{K}}$. Since $\iota_{\text {cris }, \mathbb{C}_{p} / \mathscr{K}}$ commutes with the projections $\vartheta$ and $\theta_{\mathbb{C}_{p} / \mathscr{K}}$, we have the commutative diagram

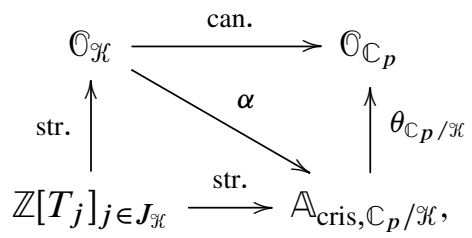

where the horizontal structure map is given by $T_{j} \mapsto t_{j}$. By Lemma 3.14(ii), $\alpha$ coincides with the structure map $\mathbb{O}_{\mathscr{K}} \rightarrow \mathbb{A}_{\text {cris, } \mathbb{C}_{p} / \mathscr{K}}$, which implies the assertion.

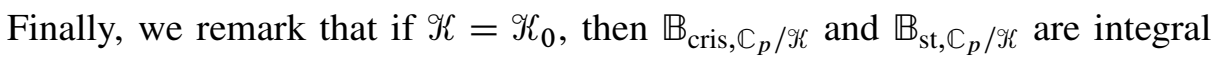
domains by the above explicit description of $\mathbb{A}_{\text {cris, }, \mathbb{C}_{p} / \mathscr{K}}$. 
3E. Connections and Frobenius on $\mathbb{B}_{\text {cris }}$ and $\mathbb{B}_{\text {st. }}$ In this section, assume $\mathscr{K}=\mathscr{K}_{0}$.

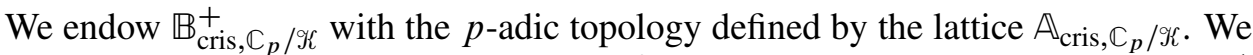
regard $\mathbb{B}_{\text {cris, }, \mathbb{C}_{p} / \mathscr{K}}$ as the direct limit of $\mathbb{B}_{\text {cris, } \mathbb{C}_{p} / \mathscr{K}}^{+}$under the multiplication by $t^{-1}$ and we set

$$
\widehat{\Omega}_{\mathscr{K}}^{q} \hat{\otimes}_{\mathscr{K}} \mathbb{B}_{\text {cris }, \mathbb{C}_{p} / \mathscr{K}}=\underline{\lim } \hat{\Omega}_{\mathscr{K}}^{q} \hat{\otimes}_{\mathscr{K}} \mathbb{B}_{\text {cris }, \mathbb{C}_{p} / \mathscr{K}}^{+} .
$$

Then, the canonical derivation $d: \mathscr{K} \rightarrow \widehat{\Omega}_{\mathscr{K}}^{1}$ uniquely extends to a $\mathbb{B}_{\text {cris }, \mathbb{C}_{p} / \mathscr{K}}$-linear continuous derivation $\nabla: \mathbb{B}_{\text {cris, } \mathbb{C}_{p} / \mathscr{K}} \rightarrow \widehat{\Omega}_{\mathscr{F}}^{1} \widehat{\otimes}_{\mathscr{K}} \mathbb{B}_{\text {cris, } \mathbb{C}_{p} / \mathscr{K}}$ by the explicit description

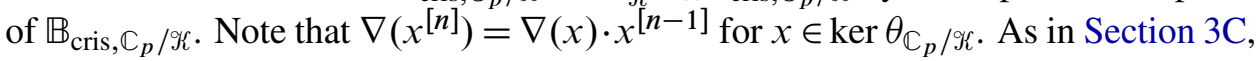
if we denote by $\left\{\partial_{j}\right\}_{j \in J_{\mathscr{K}}}$ the derivations on $\mathbb{B}_{\text {cris }, \mathbb{C}_{p} / \mathscr{K} \mathcal{K}}$ given by $\nabla(x)=\sum_{j \in J_{\mathscr{K}}} d t_{j} \otimes$

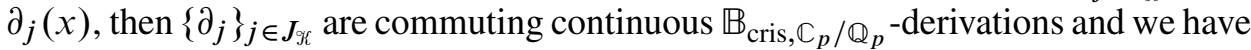
$\partial_{j}=\partial / \partial u_{j}$. We also have a canonical extension $\nabla_{q}$ of exterior derivations $d_{q}$. Also, we can uniquely extend $\nabla_{q}$ to the map $\nabla_{q}: \hat{\Omega}_{\mathscr{K}}^{q} \hat{\otimes}_{\mathscr{K}} \mathbb{B}_{\mathrm{st}, \mathbb{C}_{p} / \mathscr{K}} \rightarrow \hat{\Omega}_{\mathscr{K}}^{q+1} \hat{\otimes}_{\mathscr{K}} \mathbb{B}_{\mathrm{st}, \mathbb{C}_{p} / \mathscr{K}}$ by putting $\nabla(\mathrm{x})=0$, where we define $\widehat{\Omega}_{\mathscr{K}}^{q} \hat{\otimes}_{\mathscr{K}} \mathbb{B}_{\mathrm{st}, \mathbb{C}_{p} / \mathscr{K}}:=\left(\widehat{\Omega}_{\mathscr{K}}^{q} \hat{\otimes}_{\mathscr{K}} \mathbb{B}_{\text {cris }, \mathbb{C}_{p} / \mathscr{K}}\right)[\mathrm{x}]$.

Let $\varphi: \mathrm{O}_{\mathscr{K}} \rightarrow \mathrm{O}_{\mathscr{K}}$ be a lift of the absolute Frobenius on $k_{\mathscr{K}}$. The ring homomorphism $\varphi \otimes \varphi: \mathscr{O}_{\mathscr{K}} \otimes W\left(R_{\mathbb{C}_{p}}\right) \rightarrow \mathscr{O}_{\mathscr{K}} \otimes W\left(R_{\mathbb{C}_{p}}\right)$ induces a ring homomorphism on $\mathbb{A}_{\text {cris }, \mathbb{C}_{p} / \mathscr{K}}$. Although the resulting map depends on the choice of a Frobenius lift of $O_{\mathscr{K}}$ in general, we denote it by $\varphi$ again. By defining $\varphi(\mathrm{x}):=p \mathrm{x}$, we also have a Frobenius on $\mathbb{B}_{\mathrm{st}, \mathbb{C}_{p} / \mathscr{r}}$. By construction, the connection and the Frobenius on $\mathbb{B}_{\text {cris, } \mathbb{C}_{p} / \mathscr{F}}$ commute and we have the relation $N \circ \varphi=p \varphi \circ N$ by a simple calculation.

Notation. We define $\mathbb{B}_{\diamond, \mathbb{C}_{p} / \mathscr{K}}^{\nabla}:=\left(\mathbb{B}_{\diamond, \mathbb{C}_{p} / \mathscr{K}}\right)^{\nabla=0}$ for $\diamond \in\{$ cris, st $\}$.

By the commutativity of $\nabla$ and $\varphi$, these rings are endowed with $\varphi$-actions. Obviously, $\mathbb{B}_{\mathrm{st}, \mathbb{C}_{p} / \mathscr{K}}^{\nabla}$ is endowed with the monodromy operator $N$. By the explicit description of $\mathbb{B}_{\text {cris, } \mathbb{C}_{p} / \mathscr{\digamma}}$, we have:

Lemma 3.15. For $\diamond \in\{$ cris, st $\}$, the canonical map

$$
\mathbb{B}_{\diamond, \mathbb{C}_{p} / \mathbb{Q}_{p}} \rightarrow \mathbb{B}_{\diamond, \mathbb{C}_{p} / \mathscr{K}}^{\nabla}
$$

is an isomorphism. Since this map is compatible with Frobenius, Frobenius on $\mathbb{B}_{\diamond, \mathbb{C}_{p} / \mathscr{K}}^{\nabla}$ is independent of the choice of a Frobenius lift of $\mathcal{O}_{\mathscr{K}}$. In particular, the Frobenius on $\mathbb{B}_{\diamond, \mathbb{C}_{p} / \mathscr{K}}^{\nabla}$ is injective.

3F. Compatibility with limit. When a $p$-basis of $k_{\mathscr{K}}$ is not finite, some technical difficulties occur. In this case, we will reduce to the finite $p$-basis case by using the results of Section $1 \mathrm{G}$ and the following inverse limits.

Let the notation be as in Section 1G. By functoriality, we have canonical maps

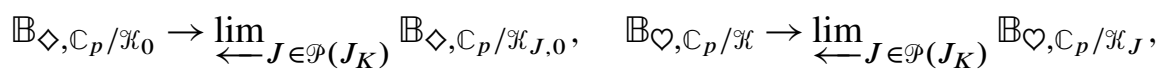

where $\diamond \in\{$ cris, st $\}, \varnothing \in\{\mathrm{dR}, \mathrm{HT}\}$. Since these morphisms are compatible with 
the above explicit descriptions of these rings, it is easy to see that these maps are injective.

3G. Embeddings of $\mathbb{B}_{\text {cris }}$ and $\mathbb{B}_{\mathrm{st}}$ into $\mathbb{B}_{\mathrm{dR}}$. Let

$$
\mathbb{J}_{\mathbb{C}_{p} / \mathscr{K}}:=\operatorname{ker}\left(\theta_{\mathbb{C}_{p} / \mathscr{K}}: \mathbb{A}_{\mathrm{inf}, \mathbb{C}_{p} / \mathscr{K}}\left[p^{-1}\right] \rightarrow \mathbb{C}_{p}\right) .
$$

We endow the ideal $\mathbb{J}_{\mathbb{C}_{p} / \mathscr{K}} / \mathbb{J}_{\mathbb{C}_{p} / \mathscr{K}}^{n}$ of the $\mathbb{Q}$-algebra $\mathbb{A}_{\text {inf, } \mathbb{C}_{p} / \mathscr{K}}\left[p^{-1}\right] / \mathbb{J}_{\mathbb{C}_{p} / \mathscr{K}}^{n}$ with the unique PD-structure. This is compatible with the canonical PD-structure of $O_{\mathscr{K}}$ on the ideal $(p)$. Hence, the canonical map $\mathcal{O}_{\mathscr{K}} \otimes_{\mathbb{Z}} W\left(R_{\mathbb{C}_{p}}\right) \rightarrow \mathbb{A}_{\text {inf, } \mathbb{C}_{p} / \mathscr{K}}\left[p^{-1}\right] / \mathbb{J}_{\mathbb{C}_{p}}^{n} / \mathscr{K}$ factors through $\left(\mathscr{O}_{\mathscr{K}} \otimes_{\mathbb{Z}} W\left(R_{\mathbb{C}_{p}}\right)\right)^{\mathrm{PD}} \rightarrow \mathbb{A}_{\text {inf, } \mathbb{C}_{p} / \mathscr{K}}\left[p^{-1}\right] / \mathbb{I}_{\mathbb{C}_{p} / \mathscr{K}}^{n}$. If we endow the LHS and the RHS with the $p$-adic topology and the $p$-adic Banach space topology respectively (see Section $3 \mathrm{~B}$ ), then the above morphism is continuous. In fact, the canonical map times $n$ ! factors through the image of $\mathbb{A}_{\mathrm{inf}, \mathbb{C}_{p} / \mathscr{K}}$. By

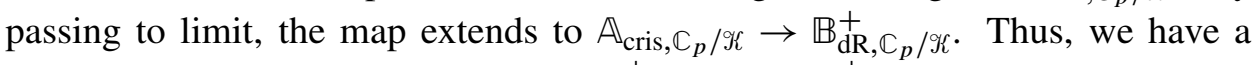

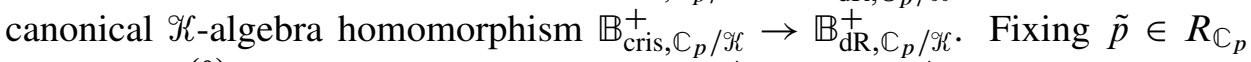
such that $\tilde{p}^{(0)}=p$, we extend this map to $\mathbb{B}_{\mathrm{st}, \mathbb{C}_{p} / \mathscr{K}}^{+} \rightarrow \mathbb{B}_{\mathrm{dR}, \mathbb{C}_{p} / \mathscr{K}}^{+}$by sending $\mathrm{x}$ to $\log ([\tilde{p}] / p):=\sum_{n \in \mathbb{N}_{>0}}(-1)^{n-1}([\tilde{p}] / p-1)^{n} / n$. Note that these morphisms are compatible with connections.

Proposition 3.16. Assume that the algebraic closure of $\mathscr{K}$ in $\mathbb{C}_{p}$ is dense in $\mathbb{C}_{p}$. Then, the canonical maps

$$
\begin{aligned}
& \mathscr{K}_{\mathrm{can}} \otimes \mathscr{K}_{\mathrm{can}, 0} \mathbb{B}_{\mathrm{cris}, \mathbb{C}_{p} / \mathscr{K}_{0}}^{\nabla} \rightarrow \mathbb{B}_{\mathrm{dR}, \mathbb{C}_{p} / \mathscr{K}}^{\nabla}, \quad \mathscr{K}_{\mathrm{can}} \otimes_{\mathscr{K}_{\mathrm{can}, 0}} \mathbb{B}_{\mathrm{st}, \mathbb{C}_{p} / \mathscr{K}_{0}}^{\nabla} \rightarrow \mathbb{B}_{\mathrm{dR}, \mathbb{C}_{p} / \mathscr{K}}^{\nabla}, \\
& \mathscr{K} \otimes_{\mathscr{K}_{0}} \mathbb{B}_{\mathrm{cris}, \mathbb{C}_{p} / \mathscr{K}_{0}} \rightarrow \mathbb{B}_{\mathrm{dR}, \mathbb{C}_{p} / \mathscr{K}}, \quad \mathscr{K} \otimes_{\mathscr{K}_{0}} \mathbb{B}_{\mathrm{st}, \mathbb{C}_{p} / \mathscr{K}_{0}} \rightarrow \mathbb{B}_{\mathrm{dR}, \mathbb{C}_{p} / \mathscr{K}}
\end{aligned}
$$

are injective.

Proof. By identifying $\mathbb{C}_{p}$ with the $p$-adic completion of $\mathscr{K}^{\text {alg }}$, we may assume $\mathscr{K}=K$. Note that if $k_{K}$ is perfect, then this is due to [Fontaine 1994a, 4.2.4]. We consider the general case. We first prove the first two cases. We have only to prove the semistable case. The canonical map $K_{\text {can }} \otimes_{K_{\text {can }, 0}} K_{0}^{\mathrm{pf}} \rightarrow K^{\mathrm{pf}}$ is injective since $K_{\text {can }} / K_{\text {can }, 0}$ is totally ramified and $K_{0}^{\mathrm{pf}}$ is absolutely unramified. Hence, we have the commutative diagram

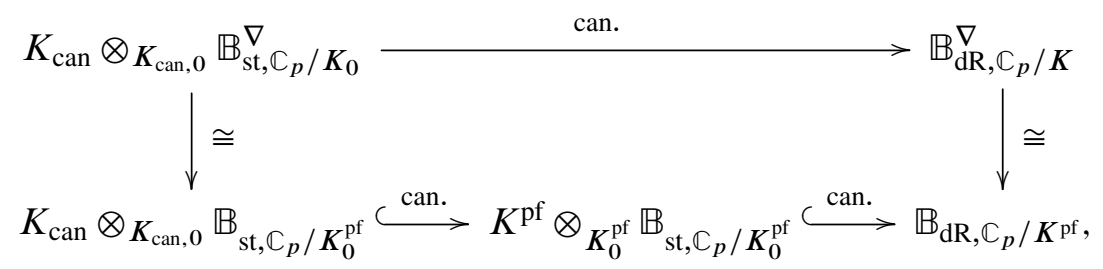

where the vertical arrows are induced by base changes and the injectivity of the bottom second arrow follows from the perfect residue field case. Then, the assertion follows from the above diagram. We consider the latter two cases. By passing 
to limit (Section $3 \mathrm{~F}$ ), we may assume $\left[k_{K}: k_{K}^{p}\right]<\infty$. Then, the crystalline case follows from [Brinon 2006, Proposition 2.47], where $\mathbb{B}_{\text {cris, } \mathbb{C}_{p} / K_{0}}$ is denoted by $\mathrm{B}_{\text {cris }}$. We will prove the semistable case. By regarding $K \otimes_{K_{0}} \mathbb{B}_{\text {cris, } \mathbb{C}_{p} / K_{0}}$ as a subring of $\operatorname{Frac}\left(\mathbb{B}_{\mathrm{dR}, \mathbb{C}_{p} / K}\right)$, the assertion is equivalent to saying that $\mathrm{x}$ is transcendental over Frac $\left(\mathbb{B}_{\text {cris, } \mathbb{C}_{p} / K_{0}}\right)$. Suppose that it is not the case. To deduce a contradiction,

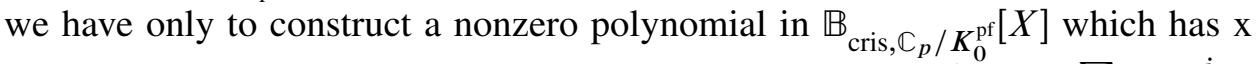
as a zero. By assumption, we have a nonzero polynomial $f(X)=\sum_{i} a_{i} X^{i} \in$ $\mathbb{B}_{\text {cris, } \mathbb{C}_{p} / K_{0}}^{+}[X]$ such that $f(\mathrm{x})=0$. For $\boldsymbol{m} \in \mathbb{N} \oplus J_{K}$, we denote by $\partial^{\boldsymbol{m}}$ the product $\prod_{j \in J_{K}} \partial_{j}^{m_{j}}$, where $\left\{\partial_{j}\right\}_{j \in J_{K}}$ are the derivations defined in Section 3C. Denote by

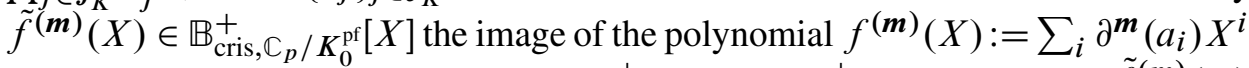

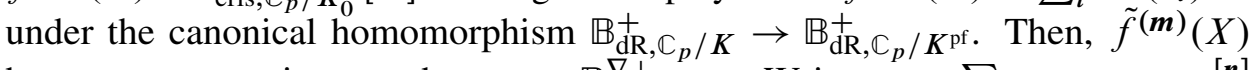
has $\mathrm{x}$ as a zero since we have $\mathrm{x} \in \mathbb{B}_{\mathrm{dR}, \mathbb{C}_{p} / K}^{\nabla+}$. Write $a_{i}=\sum_{\boldsymbol{n} \in \mathbb{N}^{\oplus J_{K}}} a_{i, \boldsymbol{n}} \boldsymbol{u}^{[\boldsymbol{n}]}$

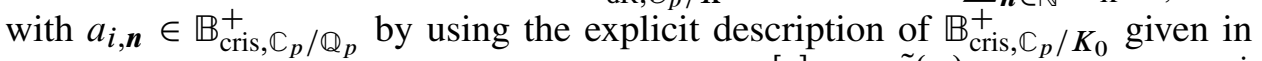
Section 3D. We have $\partial^{\boldsymbol{m}}\left(a_{i}\right)=\sum_{\boldsymbol{n} \in \mathbb{N} \oplus \nVdash} a_{i, \boldsymbol{n}+\boldsymbol{m}} \boldsymbol{u}^{[\boldsymbol{n}]}$ and $\tilde{f}^{(\boldsymbol{m})}(X)=\sum_{i} a_{i, \boldsymbol{m}} X^{i}$. Hence, we obtain the desired polynomial $\tilde{f}^{(\boldsymbol{m})}(X)$ by choosing $\boldsymbol{m} \in \mathbb{N} \oplus J_{K}$ such that we have $a_{i, \boldsymbol{m}} \neq 0$ for some $i$.

\section{Basic properties of rings of $p$-adic periods}

We will apply the preceding construction to the cases $\mathscr{K}=\mathbb{Q}_{p}, K, K^{\mathrm{pf}}$, among others. The resulting rings of $p$-adic periods will have an appropriate Galois action by the functoriality of the construction: For example, $G_{K}$ acts on $\mathbb{B}_{\mathrm{dR}, \mathbb{C}_{p} / \mathbb{Q}_{p}}$ and $\mathbb{B}_{\mathrm{dR}, \mathbb{C}_{p} / K}, G_{K^{\text {pf }}}$ acts on $\mathbb{B}_{\mathrm{dR}, \mathbb{C}_{p} / K^{\text {pr. }}}$ In this section, we will review Galois theoretic properties of these rings. The proofs of the properties are somewhat technical and the reader may skip this section by admitting the results including the $G_{K}$-regularities just below. We keep the notation of the previous section.

4A. Calculations of $\boldsymbol{H}^{0}$ and verification of $\boldsymbol{G}_{K}$-regularity. In this subsection, we will prove the $G_{K}$-regularity of the $\left(\mathbb{Q}_{p}, G_{K}\right)$-rings

$$
\begin{array}{llll}
\mathbb{B}_{\text {cris }, \mathbb{C}_{p} / K_{0}}, & \mathbb{B}_{\mathrm{st}, \mathbb{C}_{p} / K_{0},} & \mathbb{B}_{\mathrm{dR}, \mathbb{C}_{p} / K}, & \mathbb{B}_{\mathrm{HT}, \mathbb{C}_{p} / K}, \\
\mathbb{B}_{\text {cris }, \mathbb{C}_{p} / K_{0}}^{\nabla} & \mathbb{B}_{\mathrm{st}, \mathbb{C}_{p} / K_{0},}^{\nabla} & \mathbb{B}_{\mathrm{dR}, \mathbb{C}_{p} / K}^{\nabla}, & \mathbb{B}_{\mathrm{HT}, \mathbb{C}_{p} / K}^{\nabla},
\end{array}
$$

which are used later in the paper, and calculate their $H^{0}$. Note that these rings are integral domains by their explicit description.

Lemma 4.1. Let $\oslash \in\{\mathrm{dR}, \mathrm{HT}\}$.

(i) $H^{0}\left(G_{K}, \operatorname{Frac}\left(\mathbb{B}_{\bigcirc}, \mathbb{C}_{p} / K\right)\right)=K$.

(ii) The $\left(\mathbb{Q}_{p}, G_{K}\right)$-ring $\mathbb{B}_{\bigcirc, \mathbb{C}_{p} / K}$ satisfies condition $\left(G \cdot R_{3}\right)$ of Section $1 H$.

(iii) The $\left(\mathbb{Q}_{p}, G_{K}\right)$-ring $\mathbb{B}_{\mathcal{O}, \mathbb{C}_{p} / K}$ is $G_{K}$-regular. 
Proof. Assertion (iii) follows from (i), (ii) and Lemma 1.21. We will prove (i) and (ii) separately in the Hodge-Tate case and the de Rham case.

(a) The Hodge-Tate case: We first verify (i). By Theorem 1.15, we have only to prove that if we have nonzero $x, y \in \mathbb{B}_{\mathrm{HT}, \mathbb{C}_{p} / K}$ such that $g(x) y=x g(y)$ for all $g \in G_{K}$, then we have $x / y \in \mathbb{C}_{p}$. We first consider the case $\left|J_{K}\right|<\infty$. Note that $\mathbb{B}_{\mathrm{HT}, \mathbb{C}_{p} / K} \cong \mathbb{C}_{p}\left[t, t^{-1},\left\{v_{j}\right\}_{j \in J_{K}}\right]$ is a uniquely factorization domain. Hence we may assume that $x$ and $y$ are relatively prime by dividing $x$ and $y$ by their greatest common divisor. Then we have $g(x)=c_{g} x$ and $g(y)=c_{g} y$ for $c_{g} \in\left(\mathbb{B}_{\mathrm{HT}, \mathbb{C}_{p} / K}\right)^{\times} \cong \bigcup_{n \in \mathbb{Z}} \mathbb{C}_{p}^{\times} t^{n}$ by assumption. By the explicit description of $\mathbb{B}_{\mathrm{HT}, \mathbb{C}_{p} / K}$, we can choose $\boldsymbol{n} \in \mathbb{N}^{J_{K}}$ such that

$$
\partial^{\boldsymbol{n}}(x) \in \mathbb{B}_{\mathrm{HT}, \mathbb{C}_{p} / K}^{\nabla} \backslash\{0\} \cong \mathbb{C}_{p}\left[t, t^{-1}\right] \backslash\{0\},
$$

where $\partial_{j}=t \partial / \partial v_{j}$ and $\partial^{\boldsymbol{n}}:=\prod_{j} \partial_{j}^{n_{j}}$ (Remark 3.8). Write $\partial^{\boldsymbol{n}}(x)=\sum_{n \in \mathbb{Z}} a_{n} t^{n}$ with $a_{n} \in \mathbb{C}_{p}$. Then, we have $g\left(\partial^{\boldsymbol{n}}(x)\right)=c_{g} \partial^{\boldsymbol{n}}(x)$ by the commutativity of $\partial_{j}$ and the $G_{K}$-action. Since $c_{g}$ is homogeneous with respect to $t$, we have $c_{g} \in \mathbb{C}_{p}$ by comparing degrees. By comparing the leading terms, we have $c_{g}=g\left(a_{n}\right) / a_{n} \chi^{n}(g)$ for all $g \in G_{K}$, where $n$ is the degree of $\partial^{\boldsymbol{n}}(x)$ with respect to $t$. Hence, we have $x / a_{n} t^{n} \in\left(\mathbb{B}_{\mathrm{HT}, \mathbb{C}_{p} / K}\right)^{G_{K}}$. Note that we have $\left(\mathbb{B}_{\mathrm{HT}, \mathbb{C}_{p} / K}\right)^{G_{K}}=K$. This follows from the facts that we have $\mathbb{B}_{\mathrm{HT}, \mathbb{C}_{p} / K}=\bigcup_{r \in \mathbb{N}} t^{-r} \mathbb{C}_{p}\left[t,\left\{t v_{j}\right\}_{j \in J_{K}}\right]$ and

$$
H^{0}\left(G_{K}, t^{-r} \mathbb{C}_{p}\left[t,\left\{t v_{j}\right\}_{j \in J_{K}}\right]\right)=K
$$

by [Brinon 2006, Lemme 2.15], where $\mathbb{C}_{p}\left[t,\left\{t v_{j}\right\}_{j \in J_{K}}\right]$ is written $\bigoplus_{r \in \mathbb{N}} \operatorname{gr}^{r}\left(\mathbb{B}_{\mathrm{dR}}^{+}\right)$ in the reference. Thus, we have $x \in \mathbb{C}_{p}^{\times} t^{n}$. By the same argument, we have $y \in \mathbb{C}_{p}^{\times} t^{m}$ for some $m \in \mathbb{Z}$. Write $x=a t^{n}, y=b t^{m}$ with $a, b \in \mathbb{C}_{p}^{\times}$. Then, we have

$$
g(a / b)=\chi^{m-n}(g)(a / b)
$$

for $g \in G_{K}$. Since $H^{0}\left(G_{K}, \mathbb{C}_{p}(n-m)\right)$ is nonzero if and only if $n=m$ by Theorem 1.15, we must have $n=m$. In particular, we have $x / y=a / b \in \mathbb{C}_{p}$.

We consider the general case. Recall the notation in Section 1G. Let $J \in \mathscr{P}\left(J_{K}\right)$ and denote by $x_{J}, y_{J}$ the image of $x, y$ in $\mathbb{B}_{\mathrm{HT}, \mathbb{C}_{p} / K_{J}}$. By applying the above result to $J_{K}=J$, if $x_{J}$ and $y_{J}$ are nonzero, then there exists $\lambda_{J} \in \mathbb{C}_{p}^{\times}$such that $x_{J}=\lambda_{J} y_{J}$. Since this $\lambda_{J}$ is uniquely determined, $\lambda=\lambda_{J}$ is independent of the choice of $J$. Since $S_{x, y}:=\left\{J \in \mathscr{P}\left(J_{K}\right) \mid x_{J} \neq 0\right.$ and $\left.y_{J} \neq 0\right\}$ is a cofinal subset of $\mathscr{P}\left(J_{K}\right)$ by the explicit description of $\mathbb{B}_{\mathrm{HT}, \mathbb{C}_{p} / K}$, we have $x=\lambda y$ by the injection in Section $1 \mathrm{G}$.

We will verify (ii). Let $x \in \mathbb{B}_{\mathrm{HT}, \mathbb{C}_{p} / K}$ be a generator of a $G_{K}$-stable $\mathbb{Q}_{p}$-line in $\mathbb{B}_{\mathrm{HT}, \mathbb{C}_{p} / K}$. Write $g(x)=c_{g} x$ with $c_{g} \in \mathbb{Q}_{p}^{\times}$. We use the same notation as above. By a similar argument as above, if $x_{J} \neq 0$, then we have $x_{J}=a_{J} t^{n_{J}}$ for $a_{J} \in \mathbb{C}_{p}^{\times}$ and $n_{J} \in \mathbb{N}$. Moreover, $a_{J}$ and $n_{J}$ are unique. In particular, $\left\{a_{J}\right\}$ and $\left\{n_{J}\right\}$ are 
constant on the cofinal subset $S_{x, x}$ of $\mathscr{P}\left(J_{K}\right)$ and we have $x \in \mathbb{C}_{p}^{\times} t^{n} \subset\left(\mathbb{B}_{\mathrm{HT}, \mathbb{C}_{p} / K}\right)^{\times}$ by the injection in Section $1 \mathrm{G}$.

(b) The de Rham case: To prove assertion (i), we have only to prove that if we have nonzero $x, y \in \mathbb{B}_{\mathrm{dR}, \mathbb{C}_{p} / K}$ such that $g(x) y=x g(y)$ for all $g \in G_{K}$, then we have $x / y \in K$. Let $J \in \mathscr{P}\left(J_{K}\right)$ and denote by $x_{J}, y_{J} \in \mathbb{B}_{\mathrm{dR}, \mathbb{C}_{p} / K_{J}}$ the image of $x, y$. If $x_{J} \neq 0$ and $y_{J} \neq 0$, then we have $x_{J} / y_{J} \in H^{0}\left(G_{K_{J}}, \operatorname{Frac}\left(\mathbb{B}_{\mathrm{dR}, \mathbb{C}_{p} / K_{J}}\right)\right)=K_{J}$ by

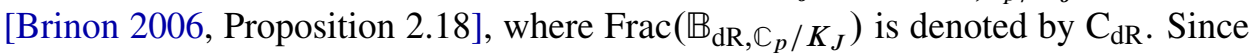
the set $\left\{J \in \mathscr{P}\left(J_{K}\right) \mid x_{J} \neq 0\right.$ and $\left.y_{J} \neq 0\right\}$ is a cofinal subset of $\mathscr{P}\left(J_{K}\right)$ by the explicit description of $\mathbb{B}_{\mathrm{dR}, \mathbb{C}_{p} / K}^{+}$, we have $x / y \in \bigcap_{J \in \mathscr{P}\left(J_{K}\right)} K_{J}=K$ by the injection in Section $1 \mathrm{G}$. We will verify (ii). By Remark 3.5(i), we may assume $K=K^{\mathrm{ur}}$. Let $V$ be a $G_{K}$-stable $\mathbb{Q}_{p}$-line in $\mathbb{B}_{\mathrm{dR}, \mathbb{C}_{p} / K}$ generated by $x$. By Lemma 4.2 below and Theorem 2.1, there exist $n \in \mathbb{Z}$ and a finite extension $L / K$ such that $V t^{n} \subset$ $\left(\mathbb{B}_{\mathrm{dR}, \mathbb{C}_{p} / K}\right)^{G_{L}}=\left(\mathbb{B}_{\mathrm{dR}, \mathbb{C}_{p} / L}\right)^{G_{L}}=L$; in particular, we have $x \in\left(\mathbb{B}_{\mathrm{dR}, \mathbb{C}_{p} / K}\right)^{\times}$.

Lemma 4.2. Let $V$ be a $G_{K}$-stable $\mathbb{Q}_{p}$-line in $\mathbb{B}_{\mathrm{dR}, \mathbb{C}_{p} / K}$. Then, up to a Tate twist, $V$ is $\mathbb{C}_{p}$-admissible as a p-adic representation.

Proof. We assume $K=K^{\mathrm{ur}}$ by Hilbert 90 and Remark 3.6(ii). Let $x \in \mathbb{B}_{\mathrm{dR}, \mathbb{C}_{p} / K}$ be a generator of $V$. By multiplying by a power of $t$, we may assume $x \in \mathbb{B}_{\mathrm{dR}, \mathbb{C}_{p} / K}^{+}$. Let $\rho: G_{K} \rightarrow \mathbb{Q}_{p}^{\times}$be the character defined by $\rho(g)=g(x) / x$. By the explicit description of $\mathbb{B}_{\mathrm{dR}, \mathbb{C}_{p} / K}^{+}$(Section 3B), we have

$$
x=\sum_{n \in \mathbb{N} \oplus J_{K}} a_{n} u^{n}
$$

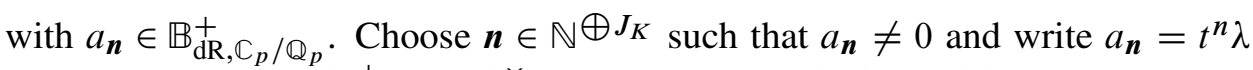
with $n \in \mathbb{N}$ and $\lambda \in\left(\mathbb{B}_{\mathrm{dR}, \mathbb{C}_{p} / \mathbb{Q}_{p}}^{+}\right)^{\times}$. Since we have $g\left(a_{\boldsymbol{n}}\right)=\rho(g) a_{\boldsymbol{n}}$ for $g \in G_{K^{\mathrm{pf}}}$, we have $\left(\rho \chi^{-n}\right)(g)=g(\lambda) / \lambda$ for $g \in G_{K^{\text {pf }}}$. By taking the $\mathbb{Q}_{p}$-linear map $\theta_{\mathbb{C}_{p} / \mathbb{Q}_{p}}$, we have $\left(\rho \chi^{-n}\right)(g)=g\left(\theta_{\mathbb{C}_{p} / \mathbb{Q}_{p}}(\lambda)\right) / \theta_{\mathbb{C}_{p} / \mathbb{Q}_{p}}(\lambda)$ for $g \in G_{K^{\mathrm{pf}}}$, that is, $\left.\rho \chi^{-n}\right|_{K^{\mathrm{pf}}}$ is $\mathbb{C}_{p^{-}}$-admissible. Hence, $\rho \chi^{-n}$ is $\mathbb{C}_{p}$-admissible by Theorem 2.1.

Corollary 4.3. We have

$$
\begin{gathered}
\left(\mathbb{B}_{\mathrm{cris}, \mathbb{C}_{p} / K_{0}}^{\nabla}\right)^{G_{K}}=\left(\mathbb{B}_{\mathrm{st}, \mathbb{C}_{p} / K_{0}}^{\nabla}\right)^{G_{K}}=K_{\mathrm{can}, 0}, \\
\left(\mathbb{B}_{\mathrm{cris}, \mathbb{C}_{p} / K_{0}}\right)^{G_{K}}=\left(\mathbb{B}_{\mathrm{st}, \mathbb{C}_{p} / K_{0}}\right)^{G_{K}}=K_{0}, \\
\left(\mathbb{B}_{\mathrm{dR}, \mathbb{C}_{p} / K}^{\nabla+}\right)^{G_{K}}=\left(\mathbb{B}_{\mathrm{dR}, \mathbb{C}_{p} / K}^{\nabla}\right)^{G_{K}}=K_{\mathrm{can}}, \\
\left(\mathbb{B}_{\mathrm{dR}, \mathbb{C}_{p} / K}^{+}\right)^{G_{K}}=\left(\mathbb{B}_{\mathrm{dR}, \mathbb{C}_{p} / K}\right)^{G_{K}}=K, \\
\left(\mathbb{B}_{\mathrm{HT}, \mathbb{C}_{p} / K}^{\nabla}\right)^{G_{K}}=\left(\mathbb{B}_{\mathrm{HT}, \mathbb{C}_{p} / K}\right)^{G_{K}}=K .
\end{gathered}
$$

Proof. Since we have trivial inclusions (such as $K_{0} \subset\left(\mathbb{B}_{\text {cris, } \mathbb{C}_{p} / K_{0}}\right)^{G_{K}}$ ), we have only to show the converse inclusions. By passing to limit (Section $1 \mathrm{G}$ and $3 \mathrm{~F}$ ), we may assume $\left[k_{K}: k_{K}^{p}\right]<\infty$. We prove the Hodge-Tate case first. Since we 
have $\mathbb{B}_{\mathrm{HT}, \mathbb{C}_{p} / K}^{\nabla} \cong \bigoplus_{n \in \mathbb{Z}} \mathbb{C}_{p}(n)$ (Section 3B), the assertion for $\mathbb{B}_{\mathrm{HT}, \mathbb{C}_{p} / K}^{\nabla}$ follows from Theorem 1.15. The assertion for $\mathbb{B}_{\mathrm{HT}, \mathbb{C}_{p} / K}$ follows from [Brinon 2006, Lemme 2.15].

We will prove the rest of the assertion. Since we have $K_{\text {can }, 0}=\left(K_{0}\right)_{\text {can }}$ by comparing the residue fields, the assertions in the horizontal case follow from those in the $\nabla$-less case by taking horizontal sections. The de Rham case follows from Lemma 4.1(i) and the crystalline and semistable cases follow from de Rham case and Proposition 3.16.

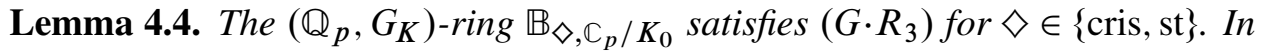

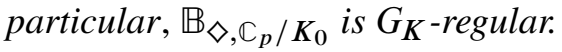

Proof. Note that the last assertion is obtained by applying Lemma 1.20, whose assumptions are satisfied by Proposition 3.16, Lemma 4.1(iii) and Corollary 4.3. By Remark 3.12(ii), we may assume $K=K^{\text {ur }}$. Let $V$ be a $G_{K}$-stable $\mathbb{Q}_{p}$-line in $\mathbb{B}_{\diamond, \mathbb{C}_{p} / K_{0}}$ with generator $x$. By Lemma 4.2, there exists $n \in \mathbb{Z}$ such that $V t^{n}$ is $\mathbb{C}_{p}$-admissible as a $p$-adic representation of $G_{K}$. By Theorem 2.1, the image of the map $\rho: G_{K} \rightarrow \mathbb{Q}_{p}^{\times}$that takes $g$ to $g\left(x t^{n}\right) /\left(x t^{n}\right)$ is included in $\left(\mathbb{Q}_{p}^{\times}\right)_{\text {tors }}$, which is killed by $2(p-1)$. Therefore, we have $\left(x t^{n}\right)^{2(p-1)} \in\left(\mathbb{B}_{\diamond, \mathbb{C}_{p} / K_{0}}\right)^{G_{K}}=K_{0}$, which implies $x \in \mathbb{B}_{\diamond, \mathbb{C}_{p} / K_{0}}^{\times}$

Lemma 4.5. The $\left(\mathbb{Q}_{p}, G_{K}\right)$-rings

$$
\mathbb{B}_{\mathrm{cris}, \mathbb{C}_{p} / K_{0}}^{\nabla}, \quad \mathbb{B}_{\mathrm{st}, \mathbb{C}_{p} / K_{0}}^{\nabla}, \quad \mathbb{B}_{\mathrm{dR}, \mathbb{C}_{p} / K}^{\nabla}, \quad \mathbb{B}_{\mathrm{HT}, \mathbb{C}_{p} / K}^{\nabla}
$$

are $G_{K}$-regular.

Proof. The $G_{K}$-regularity of the field $\mathbb{B}_{\mathrm{dR}, \mathbb{C}_{p} / K}^{\nabla}$ follows from Example 1.18.

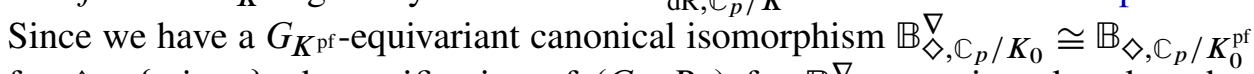
for $\diamond \in\{$ cris, st $\}$, the verification of $\left(G \cdot R_{3}\right)$ for $\mathbb{B}_{\diamond, \mathbb{C}_{p} / K_{0}}^{\nabla}$ is reduced to that

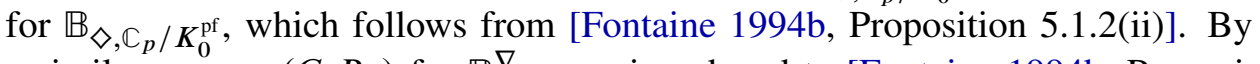
a similar reason, $\left(G \cdot R_{3}\right)$ for $\mathbb{B}_{\mathrm{dR}, \mathbb{C}_{p} / K}^{\nabla}$ is reduced to [Fontaine 1994b, Proposition 3.6]. The $\left(\mathbb{Q}_{p}, G_{K}\right)$-ring $\mathbb{C}_{p}((t))$ is a field containing the fractional field of $\mathbb{B}_{\mathrm{HT}, \mathbb{C}_{p} / K}^{\nabla} \cong \mathbb{C}_{p}\left[t, t^{-1}\right]$. By Theorem 1.15 and dévissage, we have $\mathbb{C}_{p}((t)){ }^{G_{K}}=$ $K=\left(\mathbb{B}_{\mathrm{HT}, \mathbb{C}_{p} / K}\right)^{G_{K}}$, where the last equality follows from Corollary 4.3. By applying Lemma $1.21, \mathbb{B}_{\mathrm{HT}, \mathbb{C}_{p} / K}^{\nabla}$ is $G_{K}$-regular. By Corollary 4.3 , the $G_{K}$-regularity for $\mathbb{B}_{\text {cris, }, \mathbb{C}_{p} / K_{0}}^{\nabla}$ and $\mathbb{B}_{\mathrm{st}, \mathbb{C}_{p} / K_{0}}^{\nabla}$ follows from Lemma 1.20 and Proposition 3.16.

Remark 4.6. For $\bullet \in\{$ cris, st, dR, HT $\}$, the $\left(\mathbb{Q}_{p}, G_{K}\right)$-rings $\mathbb{B}_{\bullet}^{\nabla}, \mathbb{C}_{p} / \mathbb{Q}_{p}$ and $\mathbb{B}_{\bullet}, \mathbb{C}_{p} / \mathbb{Q}_{p}$ are $G_{K}$-regular We also have

$$
\left.\left(\mathbb{B}_{\bullet, \mathbb{C}_{p} / \mathbb{Q}_{p}}^{\nabla}\right)^{G_{K}}=\left(\mathbb{B}_{\bullet}, \mathbb{C}_{p} / \mathbb{Q}_{p}\right)\right)^{G_{K}} \cong\left(\mathbb{B}_{\bullet, \mathbb{C}_{p} / K_{0}}^{\nabla}\right)^{G_{K}} .
$$

In fact, the assertion follows from canonical isomorphisms $\mathbb{B}_{\bullet}^{\nabla} \mathbb{C}_{p} / \mathbb{Q}_{p}=\mathbb{B}_{\bullet}, \mathbb{C}_{p} / \mathbb{Q}_{p} \rightarrow$ $\mathbb{B}_{\bullet}^{\nabla} \mathbb{C}_{p} / K_{0}$ as $\left(\mathbb{Q}_{p}, G_{K}\right)$-rings. 
Notation. (i) We define the category of crystalline (resp. horizontal crystalline) representations of $G_{K}$ as $\operatorname{Rep}_{\mathbb{B}_{\text {cris, } \mathbb{C}_{p} / K_{0}}^{\text {adm }}} G_{K}$ (resp. $\operatorname{Rep}_{\mathbb{B}_{\text {cris, } \mathbb{C}_{p} / K_{0}}^{\mathrm{adm}}}^{\text {adm }} G_{K}$ ), and we denote it by $\operatorname{Rep}_{\text {cris }} G_{K}$ (resp. $\operatorname{Rep}_{\text {cris }}^{\nabla} G_{K}$ ). The corresponding functor $\mathbb{D}_{B}$ is denoted by $\mathbb{D}_{\text {cris }}\left(\right.$ resp. $\mathbb{D}_{\text {cris }}^{\nabla}$ ) and the comparison map $\alpha_{B}$ by $\alpha_{\text {cris, }, \mathbb{C}_{p} / K_{0}}\left(\right.$ resp. $\alpha_{\text {cris, } \mathbb{C}_{p} / K_{0}}^{\nabla}$ ). We define the category of semistable representations similarly, with "cris" in place of "st".

(ii) We define the category of de Rham (resp. horizontal de Rham) representations of $G_{K}$ as $\operatorname{Rep}_{\mathbb{B}_{\mathrm{dR}, \mathbb{C}_{p} / K}}^{\mathrm{adm}} G_{K}$ (resp. $\operatorname{Rep}_{\mathbb{B}_{\mathrm{dR}, \mathbb{C}_{p} / K}^{\mathrm{adm}}}^{\mathrm{adm}} G_{K}$ ), and we denote it by $\operatorname{Rep}_{\mathrm{dR}} G_{K}$ (resp. $\operatorname{Rep}_{\mathrm{dR}}^{\nabla} G_{K}$ ). The corresponding functor $\mathbb{D}_{B}$ is denoted by $\mathbb{D}_{\mathrm{dR}}$ (resp. $\mathbb{D}_{\mathrm{dR}}^{\nabla}$ ) and the comparison map $\alpha_{B}$ (loc. cit.) by $\alpha_{\mathrm{dR}, \mathbb{C}_{p} / K}$ (resp. $\alpha_{\mathrm{dR}, \mathbb{C}_{p} / K}^{\nabla}$ ). We define the category of Hodge-Tate representations similarly, with "dR" in place of "HT".

(iii) We define rings with $G_{K}$-actions and automorphisms $\varphi$ by

$$
\tilde{\mathbb{B}}_{\mathrm{rig}, \mathbb{C}_{p} / K_{0}}^{\nabla+}:=\bigcap_{n \in \mathbb{N}} \varphi^{n}\left(\mathbb{B}_{\text {cris, } \mathbb{C}_{p} / K_{0}}^{\nabla+}\right), \quad \tilde{\mathbb{B}}_{\log , \mathbb{C}_{p} / K_{0}}^{\nabla+}:=\bigcap_{n \in \mathbb{N}} \varphi^{n}\left(\mathbb{B}_{\mathrm{st}, \mathbb{C}_{p} / K_{0}}^{\nabla+}\right) .
$$

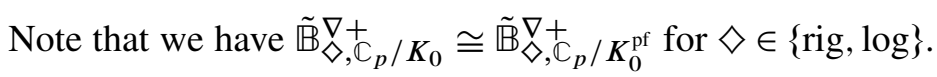

(iv) In the rest of the paper, when $k_{K}$ is perfect, we omit hyperscripts $\nabla$ to be

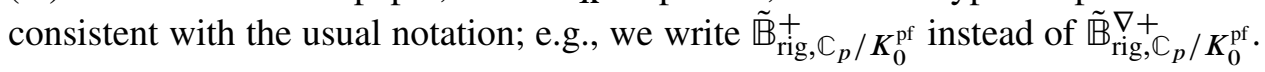

Remark 4.7. As is explained in Section $1 \mathrm{~A}$, there is no canonical choice of a Cohen ring of $k_{K}$ nor a Frobenius lift when $k_{K}$ is not perfect. Since some definitions, such as the definition of crystalline representations, involve these choices, we make some remarks on the independence of definitions.

(i) Since we have a canonical isomorphism $\mathbb{B}_{\mathcal{O}, \mathbb{C}_{p} / \mathbb{Q}_{p}} \cong \mathbb{B}_{\mathcal{O}, \mathbb{C}_{p} / K}^{\nabla}$ for $\odot \in\{\mathrm{dR}, \mathrm{HT}\}$ (Lemma 3.7), $\mathbb{B}_{\odot, \mathbb{C}_{p} / K}^{\nabla}$ depend only on $\mathbb{C}_{p}$ as an abstract ring.

(ii) Since we have a canonical isomorphism $\mathbb{B}_{\diamond, \mathbb{C}_{p} / \mathbb{Q}_{p}}^{+} \cong \mathbb{B}_{\diamond, \mathbb{C}_{p} / K_{0}}^{\nabla+}$ for $\diamond \in\{$ cris, st $\}$ (Lemma 3.15), the category $\operatorname{Rep}_{\diamond}^{\nabla} G_{K}$ depends only on $\mathbb{C}_{p}$ but not on the choice of $K_{0}$. It also follows that $\tilde{\mathbb{B}}_{\diamond, \mathbb{C}_{p} / K_{0}}^{\nabla}$ for $\diamond \in\{$ rig, log\} is independent of the choices of $K_{0}$ and $\varphi$ as a $\mathbb{Q}_{p}$-algebra with $\varphi$-action. Moreover, for a finite extension $L / K, \tilde{\mathbb{B}}_{\diamond, \mathbb{C}_{p} / K_{0}}^{\nabla+}$ coincides with $\tilde{\mathbb{B}}_{\diamond, \mathbb{C}_{p} / L_{0}}^{\nabla}$ in $\mathbb{B}_{\mathrm{dR}, \mathbb{C}_{p} / L}^{\nabla+}$

(iii) By definition, the category $\operatorname{Rep}_{\diamond} G_{K}$ for $\diamond \in\{$ cris, st $\}$ may depend on the choice of $K_{0}$. In the case $\left[k_{K}: k_{K}^{p}\right]<\infty$ with $\diamond=$ cris, the independence is proved by Brinon [2006, Proposition 3.42]: He proves the assertion by introducing a ring $A_{\max , K}$, which is independent of the choice of $K_{0}$ and is slightly bigger than $\mathbb{O}_{K} \otimes_{\mathcal{O}_{K_{0}}} \mathbb{A}_{\text {cris, } \mathbb{C}_{p} / K_{0}}$. Although a similar idea seems to work in the general case, we do not treat this problem in this paper. Instead, we will state a precise version of the Main Theorem later (see Section 6).

Remark 4.8 (Hilbert 90). Let $V \in \operatorname{Rep}_{\mathbb{Q}_{p}} G_{K}$. Then, $V$ is crystalline or semistable if

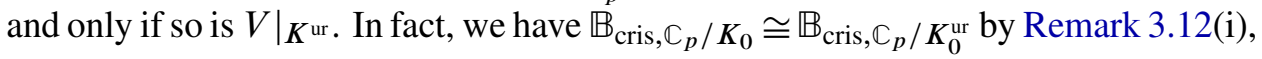


whose $G_{K}$ ur-invariant is $K_{0}^{\text {ur }}$ by Corollary 4.3 . Hence, the assertion in the crystalline case follows from Hilbert 90 and the same proof works also in the semistable case. We can also prove that $V$ is de Rham or Hodge-Tate if and only if so is $\left.V\right|_{L}$ for a finite extension $L$ of the completion of an unramified extension of $K$. This follows from the cases when $L / K$ is finite or unramified and in these cases the claim follows from Remark 3.6(ii) and Hilbert 90.

Algebraic structures of rings of $p$-adic period, which are compatible with the action of $G_{K}$, induce additional structures on the corresponding $\mathbb{D}$. We do not review these structures here since we do not need all of them to prove the Main Theorem. For the reader interested in these structures, see [Brinon 2006, 3.5] for example. We need only the connection on $\mathbb{D}_{\mathrm{dR}}$ for the proof of the Main Theorem: For $V \in \operatorname{Rep}_{\mathrm{dR}} G_{K}$, the finite-dimensional $K$-vector space $\mathbb{D}_{\mathrm{dR}}(V)$ has a connection $\nabla: \mathbb{D}_{\mathrm{dR}}(V) \rightarrow \widehat{\Omega}_{K}^{1} \otimes_{K} \mathbb{D}_{\mathrm{dR}}(V)$, which is compatible with the canonical derivation on $K$.

4B. Restriction to perfection. If we have $V \in \operatorname{Rep} . G_{K}$ with $\bullet \in\{$ cris, st, dR, HT $\}$, then we have $\left.V\right|_{K^{\text {pf }}} \in \operatorname{Rep} . G_{K^{\text {pf }}}$. Moreover, we have canonical isomorphisms

$$
K_{0}^{\mathrm{pf}} \otimes_{K_{0}} \mathbb{D}_{\diamond}(V) \rightarrow \mathbb{D}_{\diamond}\left(\left.V\right|_{K_{0}^{\mathrm{pf}}}\right), \quad K^{\mathrm{pf}} \otimes_{K} \mathbb{D}_{\odot}(V) \rightarrow \mathbb{D}_{\odot}\left(\left.V\right|_{K^{\mathrm{pf}}}\right),
$$

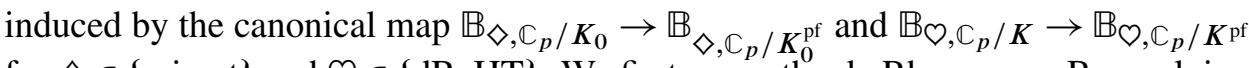
for $\diamond \in\{$ cris, st $\}$ and $\varnothing \in\{\mathrm{dR}, \mathrm{HT}\}$. We first prove the de Rham case. By applying $\mathbb{B}_{\mathrm{dR}, \mathbb{C}_{p} / K^{\mathrm{pf}}} \otimes_{\mathbb{B}_{\mathrm{dR}, \mathbb{C}_{p} / K}}$ to the comparison isomorphism $\alpha_{\mathrm{dR}, \mathbb{C}_{p} / K}(V)$, we have a $G_{K^{\mathrm{pf}}}$-equivariant isomorphism

$$
\mathbb{B}_{\mathrm{dR}, \mathbb{C}_{p} / K^{\mathrm{pf}}} \otimes_{K} \mathbb{D}_{\mathrm{dR}}(V) \rightarrow \mathbb{B}_{\mathrm{dR}, \mathbb{C}_{p} / K^{\mathrm{pf}}} \otimes_{\mathbb{Q}_{p}} V .
$$

By taking $G_{K^{\text {pf }}}$-invariant, we have an isomorphism $K^{\text {pf }} \otimes_{K} \mathbb{D}_{\mathrm{dR}}(V) \rightarrow \mathbb{D}_{\mathrm{dR}}\left(\left.V\right|_{K^{\mathrm{pf}}}\right)$. The other cases follow similarly.

\section{Construction of $\tilde{\mathbb{N}}_{\text {rig }}^{\nabla}+(V)$}

In this section, we construct a $\left(\varphi, G_{K}\right)$-module $\widetilde{\mathbb{N}}_{\text {rig }}^{\nabla+}(V)$ over $\widetilde{\mathbb{B}}_{\text {rig, }}^{\nabla+} \mathbb{C}_{p} / K_{0}$ for a de Rham representation $V$ of $G_{K}$, possibly after a Tate twist. Our $\widetilde{\mathbb{N}}_{\text {rig }}^{\nabla+}$ coincides with Colmez's $\tilde{\mathbb{N}}_{\text {rig }}^{+}$when the residue field $k_{K}$ is perfect.

We first recall Colmez's Dieudonné-Manin theorem, which is a key ingredient of

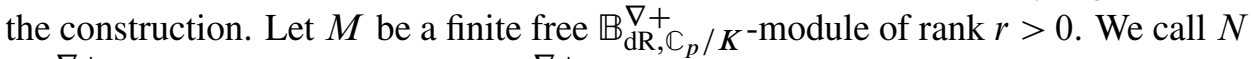
a $\mathbb{B}_{\mathrm{dR}, \mathbb{C}_{p} / K^{-}}^{\nabla+}$-lattice of $M$ if $N$ is a $\mathbb{B}_{\mathrm{dR}, \mathbb{C}_{p} / K^{-}}^{\nabla+}$-submodule of finite type of $M$ such that $N\left[t^{-1}\right]=M\left[t^{-1}\right]$. Note that a $\mathbb{B}_{\mathrm{dR}, \mathbb{C}_{p} / K^{-}}^{\nabla+}$ lattice of $M$ is finite free of rank $r$ over $\mathbb{B}_{\mathrm{dR}, \mathbb{C}_{p} / K}^{\nabla+}$ since $\mathbb{B}_{\mathrm{dR}, \mathbb{C}_{p} / K}^{\nabla+}$ is a discrete valuation ring.

For $n \in \mathbb{Z}$, denote the composition

$$
\widetilde{\mathbb{B}}_{\text {rig, }, \mathbb{C}_{p} / K_{0}}^{\nabla+} \stackrel{\varphi^{n}}{\hookrightarrow} \widetilde{\mathbb{B}}_{\text {rig, }, \mathbb{C}_{p} / K_{0}}^{\nabla+} \stackrel{\text { inc. }}{\hookrightarrow} \mathbb{B}_{\mathrm{dR}, \mathbb{C}_{p} / K}^{\nabla+}
$$


by $\varphi^{n}$ again. By the commutative diagram

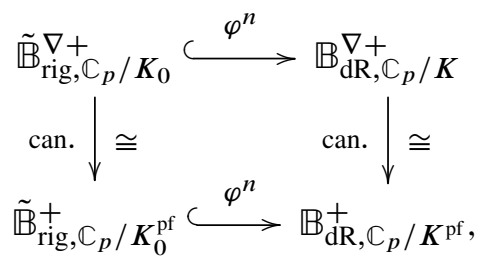

the proof of the following theorem is reduced to the perfect residue field case [Colmez 2008, Proposition 0.3] (see also the remark below).

Theorem 5.1 (Colmez's Dieudonné-Manin classification theorem). Let $r \in \mathbb{N}_{>0}$ and $M$ be a $\mathbb{B}_{\mathrm{dR}, \mathbb{C}_{p} / K}^{\nabla+}$-lattice of $\left(\mathbb{B}_{\mathrm{dR}, \mathbb{C}_{p} / K}^{\nabla+}\right)^{r}$. Let

$$
M_{\text {rig }}:=\left\{x \in\left(\widetilde{\mathbb{B}}_{\mathrm{rig}, \mathbb{C}_{p} / K_{0}}^{\nabla+}\right)^{r} \mid \varphi^{n}(x) \in M \text { for all } n \in \mathbb{Z}\right\} .
$$

Then, $M_{\text {rig }}$ is a finite free $\widetilde{\mathbb{B}}_{\text {rig, }, \mathbb{C}_{p} / K_{0}}^{\nabla+}$-module of rank $r$ with semilinear $\varphi$-action and there exists a basis $e_{1}, \ldots, e_{r}$ of $M_{\mathrm{rig}}$ over $\widetilde{\mathbb{B}}_{\mathrm{rig}, \mathbb{C}_{p} / K_{0}}^{\nabla+}$ such that:

(i) There exist $h \in \mathbb{N}_{>0}$ and $a_{1} \leq \cdots \leq a_{r} \in \mathbb{N}$ such that $\varphi^{h}\left(e_{i}\right)=p^{a_{i}} e_{i}$ for $1 \leq i \leq r$;

(ii) $e_{1}, \ldots, e_{r}$ is a basis of $M$ over $\mathbb{B}_{\mathrm{dR}, \mathbb{C}_{p} / K}^{\nabla+}$.

Remark 5.2. Though our condition (ii) is weaker than that in [Colmez 2008], the conclusions of the theorem are the same for the following reason: By definition, $\varphi$ acts on $M_{\text {rig. Since }} \varphi^{h}$ is an automorphism on $M_{\text {rig }}$ by (i), $\varphi$ is also an automorphism on $M_{\text {rig }}$. Hence, (ii) implies that $\varphi^{n}\left(e_{1}\right), \ldots, \varphi^{n}\left(e_{r}\right)$ is a $\widetilde{\mathbb{B}}_{\text {rig, } \mathbb{C}_{p} / K_{0}}^{\nabla+\text { basis of }} M_{\text {rig }}$

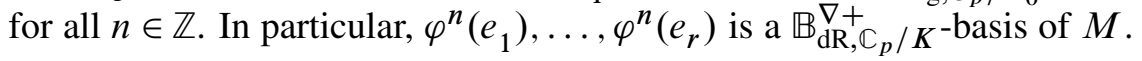

In the rest of this section, let $V$ be a de Rham representation of $G_{K}$ of dimension $r$ such that $\mathbb{D}_{\mathrm{dR}}(V)=\left(\mathbb{B}_{\mathrm{dR}, \mathbb{C}_{p} / K}^{+} \otimes_{\mathbb{Q}_{p}} V\right)^{G_{K}}$. Note that the last assumption is satisfied for any de Rham representation after some Tate twist. Let

$$
\mathbb{N}_{\mathrm{dR}}^{+}(V):=\mathbb{B}_{\mathrm{dR}, \mathbb{C}_{p} / K}^{+} \otimes_{K} \mathbb{D}_{\mathrm{dR}}(V) .
$$

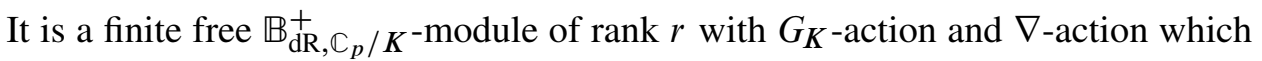
are commuting. By the comparison isomorphism $\alpha_{\mathrm{dR}, \mathbb{C}_{p} / K}$, we have a canonical isomorphism $\mathbb{N}_{\mathrm{dR}}^{+}(V)\left[t^{-1}\right] \cong \mathbb{B}_{\mathrm{dR}, \mathbb{C}_{p} / K} \otimes_{\mathbb{Q}_{p}} V$, in particular, we have

$$
t^{n} \mathbb{B}_{\mathrm{dR}, \mathbb{C}_{p} / K}^{+} \otimes_{\mathbb{Q}_{p}} V \subset \mathbb{N}_{\mathrm{dR}}^{+}(V) \subset \mathbb{B}_{\mathrm{dR}, \mathbb{C}_{p} / K}^{+} \otimes_{\mathbb{Q}_{p}} V
$$

for sufficiently large $n \in \mathbb{N}$. Taking horizontal sections, we see that $\mathbb{N}_{\mathrm{dR}}^{\nabla+}(V):=$ $\mathbb{N}_{\mathrm{dR}}^{+}(V)^{\nabla=0}$ is a $G_{K}$-stable $\mathbb{B}_{\mathrm{dR}, \mathbb{C}_{p} / K^{-}}^{\nabla+}$-lattice of $\mathbb{B}_{\mathrm{dR}, \mathbb{C}_{p} / K}^{\nabla+} \otimes_{\mathbb{Q}_{p}} V$. By applying Theorem 5.1 to $M=\mathbb{N}_{\mathrm{dR}}^{\nabla+}(V)$, we have the following proposition: (In the following, a $\left(\varphi, G_{K}\right)$-module over $\widetilde{\mathbb{B}}_{\text {rig, } \mathbb{C}_{p} / K_{0}}^{\nabla+}$ (of rank $r$ ) means a finite free module (of rank $r$ ) 
over $\tilde{\mathbb{B}}_{\text {rig, } \mathbb{C}_{p} / K_{0}}^{\nabla+}$ with a semilinear $\varphi$-action and a semilinear $G_{K}$-action, which are commuting.)

Proposition 5.3. The $\widetilde{\mathbb{B}}_{\text {rig, } \mathbb{C}_{p} / K_{0}}^{\nabla+\text { module }}$

$\tilde{\mathbb{N}}_{\text {rig }}^{\nabla+}(V):=\left\{x \in \widetilde{\mathbb{B}}_{\text {rig, }, \mathbb{C}_{p} / K_{0}}^{\nabla+} \otimes_{\mathbb{Q}_{p}} V \mid \varphi^{n} \otimes \mathrm{id}(x) \in \mathbb{N}_{\mathrm{dR}}^{\nabla+}(V)\right.$ for all $\left.n \in \mathbb{Z}\right\}$

is a $\left(\varphi, G_{K}\right)$-module over $\widetilde{\mathbb{B}}_{\text {rig, } \mathbb{C}_{p} / K_{0}}^{\nabla+}$ of rank $r$. Moreover, we have a basis $e_{1}, \ldots, e_{r}$ of $\tilde{\mathbb{N}}_{\text {rig }}^{\nabla+}(V)$ over $\widetilde{\mathbb{B}}_{\text {rig, }}^{\nabla+} \mathbb{C}_{p} / K_{0}$ such that:

(i) There exist $h \in \mathbb{N}_{>0}$ and $a_{1} \leq \cdots \leq a_{r} \in \mathbb{N}$ such that $\varphi^{h}\left(e_{i}\right)=p^{a_{i}} e_{i}$ for $1 \leq i \leq r$;

(ii) $e_{1}, \ldots, e_{r}$ is a basis of $\mathbb{N}_{\mathrm{dR}}^{\nabla+}(V)$ over $\mathbb{B}_{\mathrm{dR}, \mathbb{C}_{p} / K}^{\nabla+}$.

Note that $\tilde{\mathbb{N}}_{\text {rig }}^{\nabla+}(V)$ is independent of the choice of $K_{0}$ by Remark 4.7(ii). We will use the following property of $\tilde{\mathbb{N}}_{\text {rig }}^{\nabla+}(V)$ in the proof of the Main Theorem.

Proposition 5.4. The canonical map

$$
\mathbb{B}_{\mathrm{dR}, \mathbb{C}_{p} / K}^{+} \otimes_{\mathbb{B}_{\mathrm{dR}, \mathbb{C}_{p} / K}^{+}} \mathbb{N}_{\mathrm{dR}}^{\nabla+}(V) \rightarrow \mathbb{N}_{\mathrm{dR}}^{+}(V)
$$

is a $G_{K}$-equivariant isomorphism. In particular, $\mathbb{B}_{\mathrm{dR}, \mathbb{C}_{p} / K}^{+} \otimes_{\tilde{\mathbb{B}}_{\mathrm{rig}, \mathbb{C}_{p} / K_{0}}^{+}} \tilde{\mathbb{N}}_{\text {rig }}^{\nabla+}(V)$ is isomorphic to $\left(\mathbb{B}_{\mathrm{dR}, \mathbb{C}_{p} / K}^{+}\right)^{r}$ as a $\mathbb{B}_{\mathrm{dR}, \mathbb{C}_{p} / K}^{+}\left[G_{K}\right]$-module by Proposition 5.3(ii).

Proof. Since $\left.V\right|_{K \text { pf }}$ is de Rham and we have the canonical isomorphism $\mathbb{B}_{\mathrm{dR}, \mathbb{C}_{p} / \mathbb{Q}_{p}} \rightarrow$ $\mathbb{B}_{\mathrm{dR}, \mathbb{C}_{p} / K^{\mathrm{pf}}}$, we have the comparison isomorphism

$$
\mathbb{B}_{\mathrm{dR}, \mathbb{C}_{p} / \mathbb{Q}_{p}} \otimes_{\left(\mathbb{B}_{\mathrm{dR}, \mathbb{C}_{p} / \mathbb{Q}_{p}}\right)} G_{K^{\mathrm{pf}}}\left(\mathbb{B}_{\mathrm{dR}, \mathbb{C}_{p} / \mathbb{Q}_{p}} \otimes_{\mathbb{Q}_{p}} V\right)^{G_{K^{\mathrm{pf}}}} \rightarrow \mathbb{B}_{\mathrm{dR}, \mathbb{C}_{p} / \mathbb{Q}_{p}} \otimes_{\mathbb{Q}_{p}} V .
$$

By taking the base change of this isomorphism by $\mathbb{B}_{\mathrm{dR}, \mathbb{C}_{p} / \mathbb{Q}_{p}} \rightarrow \mathbb{B}_{\mathrm{dR}, \mathbb{C}_{p} / K}$, we obtain a canonical isomorphism of $\mathbb{B}_{\mathrm{dR}, \mathbb{C}_{p} / K}\left[G_{K^{\mathrm{pf}}}\right]$-modules

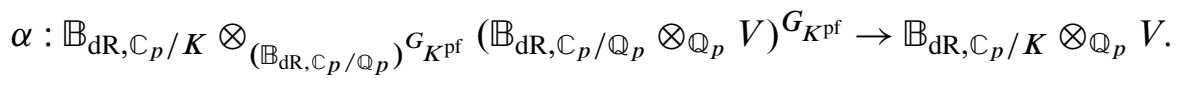

We also have the comparison isomorphism

$$
\alpha_{\mathrm{dR}, \mathbb{C}_{p} / K}(V): \mathbb{B}_{\mathrm{dR}, \mathbb{C}_{p} / K} \otimes_{K} \mathbb{D}_{\mathrm{dR}}(V) \rightarrow \mathbb{B}_{\mathrm{dR}, \mathbb{C}_{p} / K} \otimes_{\mathbb{Q}_{p}} V .
$$

Note that we have $\left(\mathbb{B}_{\mathrm{dR}, \mathbb{C}_{p} / \mathbb{Q}_{p}}^{+}\right)^{G_{K \mathrm{pf}}}=\left(\mathbb{B}_{\mathrm{dR}, \mathbb{C}_{p} / \mathbb{Q}_{p}}\right)^{G_{K^{\mathrm{pf}}}}$ since we have

$$
\left(t^{-n} \mathbb{B}_{\mathrm{dR}, \mathbb{C}_{p} / \mathbb{Q}_{p}}^{+} / t^{-n+1} \mathbb{B}_{\mathrm{dR}, \mathbb{C}_{p} / \mathbb{Q}_{p}}^{+}\right)^{G_{K^{\mathrm{pf}}}}=\left(\mathbb{C}_{p}(-n)\right)^{G_{K^{\mathrm{pf}}}}=0
$$

for $n \in \mathbb{N}_{>0}$. We have only to prove that there exists an isomorphism of $\mathbb{B}_{\mathrm{dR}, \mathbb{C}_{p} / K^{-}}^{+}$ modules

$\left(\mathbb{N}_{\mathrm{dR}}^{+}(V)=\right) \mathbb{B}_{\mathrm{dR}, \mathbb{C}_{p} / K}^{+} \otimes_{K} \mathbb{D}_{\mathrm{dR}}(V) \cong$

$$
\left.\mathbb{B}_{\mathrm{dR}, \mathbb{C}_{p} / K}^{+} \otimes_{\left(\mathbb{B}_{\mathrm{dR}, \mathbb{C}_{p} / \mathbb{Q}_{p}}\right)}\right)_{K^{\mathrm{pf}}}\left(\mathbb{B}_{\mathrm{dR}, \mathbb{C}_{p} / \mathbb{Q}_{p}} \otimes_{\mathbb{Q}_{p}} V\right)^{G_{K^{\mathrm{pf}}}}
$$

which is compatible with the injections $\alpha_{\mathrm{dR}, \mathbb{C}_{p} / K}(V)$ and $\alpha$. Indeed, by taking the 
horizontal sections of both sides, we have

$$
\left.\mathbb{N}_{\mathrm{dR}}^{\nabla+}(V)=\mathbb{B}_{\mathrm{dR}, \mathbb{C}_{p} / K}^{\nabla+} \otimes_{\left(\mathbb{B}_{\mathrm{dR}, \mathbb{C}_{p} / \mathbb{Q}_{p}}\right)}\right)_{K^{\mathrm{pf}}}\left(\mathbb{B}_{\mathrm{dR}, \mathbb{C}_{p} / \mathbb{Q}_{p}} \otimes_{\mathbb{Q}_{p}} V\right)^{G_{K^{\mathrm{pf}}}}
$$

which implies the assertion.

We have

$$
\begin{aligned}
& \mathbb{D}_{\mathrm{dR}}(V) \hookrightarrow\left(\mathbb{B}_{\mathrm{dR}, \mathbb{C}_{p} / K} \otimes_{\mathbb{Q}_{p}} V\right)^{G_{K^{\mathrm{pf}}}}= \\
& \left(\mathbb{B}_{\mathrm{dR}, \mathbb{C}_{p} / K}\right)^{G_{K \mathrm{pf}}} \otimes_{\left(\mathbb{B}_{\mathrm{dR}, \mathbb{C}_{p} / \mathbb{Q}_{p}}\right)} G_{K^{\mathrm{pf}}}\left(\mathbb{B}_{\mathrm{dR}, \mathbb{C}_{p} / \mathbb{Q}_{p}} \otimes_{\mathbb{Q}_{p}} V\right)^{G_{K^{\mathrm{pf}}}},
\end{aligned}
$$

where the equality follows by taking $G_{K^{\text {pf }}}$-invariant of (2). Note that we have

$$
\left(\mathbb{B}_{\mathrm{dR}, \mathbb{C}_{p} / K}^{+}\right)^{G_{K^{\mathrm{pf}}}}=\left(\mathbb{B}_{\mathrm{dR}, \mathbb{C}_{p} / K}\right)^{G_{K^{\mathrm{pf}}}} .
$$

Indeed, if we write $x \in$ LHS as $x=t^{-n} \sum_{n \in \mathbb{N} \oplus J_{K}} a_{\boldsymbol{n}} \boldsymbol{u}^{\boldsymbol{n}}$ with $a_{\boldsymbol{n}} \in \mathbb{B}_{\mathrm{dR}, \mathbb{C}_{p} / \mathbb{Q}_{p}}^{+}$, since $\left\{u_{j}\right\}_{j \in J_{K}}$ are invariant by the action of $G_{K}$ pf , we have

$$
b_{\boldsymbol{n}}:=a_{\boldsymbol{n}} / t^{n} \in\left(\mathbb{B}_{\mathrm{dR}, \mathbb{C}_{p} / \mathbb{Q}_{p}}\right)^{G_{K^{\mathrm{pf}}}}=\left(\mathbb{B}_{\mathrm{dR}, \mathbb{C}_{p} / \mathbb{Q}_{p}}^{+}\right)^{G_{K^{\mathrm{pf}}}} .
$$

Therefore, we have $x=\sum_{\boldsymbol{n} \in \mathbb{N} \oplus J_{K}} b_{\boldsymbol{n}} \boldsymbol{u}^{\boldsymbol{n}} \in\left(\mathbb{B}_{\mathrm{dR}, \mathbb{C}_{p} / K}^{+}\right)^{G_{K}^{\mathrm{pf}}}$. Hence we have a canonical map

$$
\mathbb{D}_{\mathrm{dR}}(V) \hookrightarrow \mathbb{B}_{\mathrm{dR}, \mathbb{C}_{p} / K}^{+} \otimes_{\left(\mathbb{B}_{\mathrm{dR}, \mathbb{C}_{p} / \mathbb{Q}_{p}}^{+}\right)}{ }^{G} K^{\mathrm{pf}}\left(\mathbb{B}_{\mathrm{dR}, \mathbb{C}_{p} / \mathbb{Q}_{p}} \otimes_{\mathbb{Q}_{p}} V\right)^{G_{K^{\mathrm{pf}}}}
$$

This induces a canonical homomorphism of $\mathbb{B}_{\mathrm{dR}, \mathbb{C}_{p} / K^{-} \text {-modules }}^{+}$

$i: \mathbb{B}_{\mathrm{dR}, \mathbb{C}_{p} / K}^{+} \otimes_{K} \mathbb{D}_{\mathrm{dR}}(V) \rightarrow \mathbb{B}_{\mathrm{dR}, \mathbb{C}_{p} / K}^{+} \otimes_{\left(\mathbb{B}_{\mathrm{dR}, \mathbb{C}_{p} / \mathbb{Q}_{p}}^{+}\right)}{ }_{K^{\mathrm{pf}}}\left(\mathbb{B}_{\mathrm{dR}, \mathbb{C}_{p} / \mathbb{Q}_{p}} \otimes_{\mathbb{Q}_{p}} V\right)^{G_{K^{\mathrm{pf}}}}$, which is compatible with the injections $\alpha_{\mathrm{dR}, \mathbb{C}_{p} / K}(V)$ and $\alpha$ by construction. We have only to prove the surjectivity of $i$. By Nakayama's lemma, we have only to prove the assertion after applying $\mathbb{B}_{\mathrm{dR}, \mathbb{C}_{p} / K^{\mathrm{pf}}}^{+} \bigotimes_{\mathbb{B}_{\mathrm{dR}, \mathbb{C}_{p} / K}^{+}}$(note that $\mathbb{B}_{\mathrm{dR}, \mathbb{C}_{p} / K}^{+} \rightarrow$

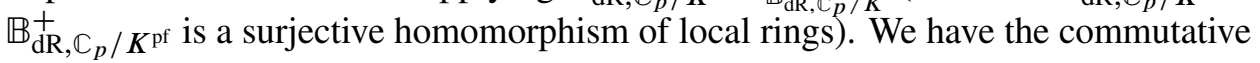
diagram

$$
\begin{aligned}
& \mathbb{B}_{\mathrm{dR}, \mathbb{C}_{p} / K^{\mathrm{pf}}}^{+} \otimes_{K} \mathbb{D}_{\mathrm{dR}}(V) \stackrel{\alpha_{\mathrm{dR}, \mathbb{C}_{p} / K}(V)_{*}}{\longrightarrow} \mathbb{B}_{\mathrm{dR}, \mathbb{C}_{p} / K^{\mathrm{pf}}} \otimes_{\mathbb{Q}_{p}} V \\
& i_{*} \downarrow
\end{aligned}
$$

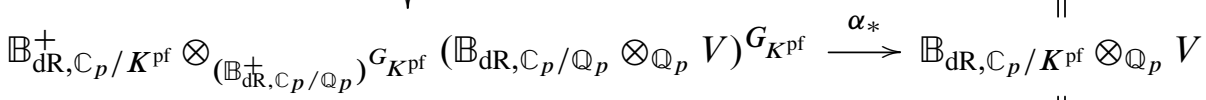

$$
\begin{aligned}
& \text { can. } ₫ \\
& \mathbb{B}_{\mathrm{dR}, \mathbb{C}_{p} / K^{\mathrm{pf}}}^{+} \otimes_{K^{\mathrm{pf}}} \mathbb{D}_{\mathrm{dR}}\left(\left.V\right|_{K^{\mathrm{pf}}}\right) \stackrel{\alpha_{\mathrm{dR}, \mathbb{C}_{p} / K^{\mathrm{pf}}}\left(\left.V\right|_{K^{\mathrm{pf}}}\right)}{\longrightarrow} \mathbb{B}_{\mathrm{dR}, \mathbb{C}_{p} / K^{\mathrm{pf}}} \otimes_{\mathbb{Q}_{p}} V,
\end{aligned}
$$

where the left lower arrow is induced by $\mathbb{B}_{\mathrm{dR}, \mathbb{C}_{p} / \mathbb{Q}_{p}} \rightarrow \mathbb{B}_{\mathrm{dR}, \mathbb{C}_{p}} / K^{\mathrm{pf}}$, the $G_{K^{\mathrm{pf}}-}$ equivariant isomorphism. Denote the composition of the left vertical arrows 
by $i^{\prime}$. Since the canonical map $\mathbb{B}_{\mathrm{dR}, \mathbb{C}_{p} / K} \rightarrow \mathbb{B}_{\mathrm{dR}, \mathbb{C}_{p} / K^{\mathrm{pf}}}$ is $G_{K^{\mathrm{pf}} \text {-equivariant, }}$ by the diagram, the restriction of $i^{\prime}$ to $\mathbb{D}_{\mathrm{dR}}(V)$ coincides with the canonical map $\mathbb{D}_{\mathrm{dR}}(V) \rightarrow \mathbb{D}_{\mathrm{dR}}\left(\left.V\right|_{K^{\mathrm{pf}}}\right.$ ), which is an isomorphism after tensoring $K^{\mathrm{pf}}$ (see Section 4B). Therefore, $i^{\prime}$ is an isomorphism and we obtain the assertion.

\section{Proof of the Main Theorem}

We will restate our main theorem in the point of view of Remark 4.7(iii):

Main Theorem. Let $V$ be a de Rham representation of $G_{K}$. Then, there exists a

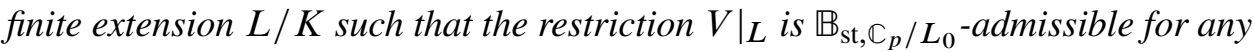
choice of $L_{0}$.

In this section, we give a proof of the Main Theorem in this form. Before the proof, we prepare technical lemmas used in the proof. The reader may go to the proof of the Main Theorem and back to the lemmas if necessary.

We first recall a slightly modified version of [Colmez 2008, Proposition 0.6]. In the rest of this section, denote the unramified extension of $\mathbb{Q}_{p}$ of degree $h \in \mathbb{N}_{>0}$ by $\mathbb{Q}_{p^{h}}$.

Proposition 6.1. Assume that $k_{K}$ is perfect. Let $\mathbb{U}_{h, a}^{\prime}:=\left(\tilde{\mathbb{B}}_{\log , \mathbb{C}_{p} / K_{0}}^{+}\right)^{\varphi^{h}=p^{a}}$ for $h$, $a \in \mathbb{N}$. Let $M$ be a $\left(\varphi, G_{K}\right)$-module over $\tilde{\mathbb{B}}_{\mathrm{rig}, \mathbb{C}_{p} / K_{0}}^{+}$of rank $r \in \mathbb{N}_{>0}$ with basis $e_{1}, \ldots, e_{r}$. Assume that there exists an isomorphism of $\mathbb{B}_{\mathrm{dR}, \mathbb{C}_{p} / K}^{+}\left[G_{K}\right]$-modules $\mathbb{B}_{\mathrm{dR}, \mathbb{C}_{p} / K}^{+} \otimes_{\tilde{\mathbb{B}}_{\mathrm{rg},}^{+}, \mathbb{C}_{p} / K} M \cong\left(\mathbb{B}_{\mathrm{dR}, \mathbb{C}_{p} / K}^{+}\right)^{r}$ and that $e_{1}, \ldots, e_{r}$ satisfies the following conditions:

(i) There exists $h \in \mathbb{N}_{>0}$ and $a_{1} \leq \cdots \leq a_{r} \in \mathbb{N}$ such that $\varphi^{h}\left(e_{i}\right)=p^{a_{i}} e_{i}$ for $1 \leq i \leq r$.

(ii) For all $g \in G_{K}$, there exists $c_{g} \in \mathrm{GL}_{r}\left(\mathbb{B}_{\mathrm{dR}, \mathbb{C}_{p} / K}^{+}\right)$, a (unique) upper triangular matrix whose diagonal entries are 1 , such that $g\left(e_{1}, \ldots, e_{r}\right)=\left(e_{1}, \ldots, e_{r}\right) c_{g}$.

Then there exists a $\tilde{\mathbb{B}}_{\log , \mathbb{C}_{p} / K_{0}}^{+}$-basis $f_{1}, \ldots, f_{r}$ of $\tilde{\mathbb{B}}_{\log , \mathbb{C}_{p} / K_{0}}^{+} \otimes_{\tilde{\mathbb{B}}_{\mathrm{rg}, \mathbb{C}_{p} / K_{0}}^{+}} M$ satisfying the following conditions:

(a) $f_{i}$ is fixed by $G_{K}$;

(b) $f_{i}=e_{i}+\sum_{1 \leq j \leq i-1} \alpha_{j i} e_{j}$ with $\alpha_{j i} \in \mathbb{U}_{h, a_{i}-a_{j}}^{\prime}$ (hence $\varphi^{h}\left(f_{i}\right)=p^{a_{i}} f_{i}$ ).

Proof. Note that we add the extra assumption (ii) and the slightly stronger conclusion (a) to the original proposition. Let $U$ be the subgroup of $\mathrm{GL}_{r}\left(\mathbb{B}_{\mathrm{dR}, \mathbb{C}_{p} / K}^{+}\right)$ consisting of upper triangular matrices whose diagonal entries are 1 and whose $(i, j)$ component belongs to $\mathbb{U}_{h, a_{j}-a_{i}}^{\prime}$ for $i<j$. We endow $U$ with the subspace topology of $\mathrm{GL}_{r}\left(\mathbb{B}_{\mathrm{dR}, \mathbb{C}_{p} / K}^{+}\right)$. Then, $U$ is a topological $G_{K}$-group and the map $g \mapsto c_{g} ; G_{K} \rightarrow U$ is a continuous 1-cocycle. By [Colmez 2008, Proposition 0.6], there exists a finite Galois extension $L / K$ such that $[c]$ is mapped to the trivial 
class in $H^{1}\left(G_{L^{\text {ur }}}, U\right)$ by the composite $\operatorname{Res}_{L}^{L}{ }^{\text {ur }} \circ \operatorname{Res}_{K}^{L}$, where $[c]$ denotes the class represented by $c$. Note that for all $a \in \mathbb{N}_{>0}$, we have

$$
\left(\mathbb{U}_{h, a}^{\prime}\right)^{G_{L} \mathrm{ur}} \subset\left(\left(\mathbb{B}_{\mathrm{st}, \mathbb{C}_{p} / L_{0}}\right)^{G_{L} \mathrm{ur}}\right)^{\varphi^{h}=p^{a}}=\left(L_{0}^{\mathrm{ur}}\right)^{\varphi^{h}=p^{a}}=0,
$$

where the first equality follows from Remark 3.12(ii) and Corollary 4.3 and the last equality follows from [Colmez 2008, Lemme 10.9]. Hence $U^{G_{L^{\text {ur }}}}=\{1\}$ and [c] is mapped to the trivial class in $H^{1}\left(G_{L}, U\right)$ by the inflation-restriction exact sequence. Hence, we have only to prove that the inverse image of the trivial element by $\operatorname{Res}_{K}^{L}: H^{1}\left(G_{K}, U\right) \rightarrow H^{1}\left(G_{L}, U\right)$ consists of the trivial element.

We endow $U$ with a $G_{K}$-stable decreasing filtration $\left\{\mathscr{F}_{n}\right\}_{n \in \mathbb{N}}$ by $\mathscr{F}_{n}:=\left\{\left(x_{i j}\right) \in\right.$ $U \mid x_{i j}=0$ for $\left.0<j-i \leq n\right\}$. Then, we have $\mathscr{F}_{0}=U, \mathscr{F}_{r}=\{1\}, \mathscr{F}_{n+1} \unlhd \mathscr{F}_{n}$ and $\mathscr{F}_{n} / \mathscr{F}_{n+1}$ is isomorphic to a direct sum of copies of $\mathbb{U}_{h, a}^{\prime}$ with $a \in \mathbb{N}$. We have only to prove that the inverse image of the trivial element under the restriction map $\operatorname{Res}_{K}^{L}: H^{1}\left(G_{K}, \mathscr{F}_{n}\right) \rightarrow H^{1}\left(G_{L}, \mathscr{F}_{n}\right)$ for $n \in \mathbb{N}$ consists of the trivial element. Since there exists a $G_{K}$-equivariant set-theoretic section of the canonical projection $\mathscr{F}_{n} \rightarrow$ $\mathscr{F}_{n} / \mathscr{F}_{n+1}$ (for example, we can identify

$$
1+\sum_{i} x_{i, i+n+1} E_{i, i+n+1} \in \mathscr{F}_{n}
$$

with its image in $\left.\mathscr{F}_{n} / \mathscr{F}_{n+1}\right)$, the canonical maps $\mathscr{F}_{n} G_{K} \rightarrow\left(\mathscr{F}_{n} / \mathscr{F}_{n+1}\right)^{G_{K}}$ and $\mathscr{F}_{n}^{G_{L}} \rightarrow\left(\mathscr{F}_{n} / \mathscr{F}_{n+1}\right)^{G_{L}}$ are surjective. By using long exact sequences, we have the commutative diagram

$$
\begin{gathered}
0 \longrightarrow H^{1}\left(G_{K}, \mathscr{F}_{n+1}\right) \stackrel{\text { can. }}{\longrightarrow} H^{1}\left(G_{K}, \mathscr{F}_{n}\right) \stackrel{\text { can. }}{\longrightarrow} H^{1}\left(G_{K}, \mathscr{F}_{n} / \mathscr{F}_{n+1}\right) \\
\downarrow \operatorname{Res}_{K}^{L}
\end{gathered}
$$

whose rows are exact as pointed sets. To prove the assertion, it suffices to prove the injectivity of the restriction map $H^{1}\left(G_{K}, \mathbb{U}_{h, a}^{\prime}\right) \rightarrow H^{1}\left(G_{L}, \mathbb{U}_{h, a}^{\prime}\right)$ for $h, a \in \mathbb{N}$. Indeed, it implies the injectivity of the right arrow in the diagram and we obtain the assertion by dévissage and diagram chasing. We first consider the case $a=0$, that is, $\mathbb{U}_{h, 0}^{\prime}=\mathbb{Q}_{p^{h}}$ (Lemma 6.2 below). Since $H^{1}\left(G_{L / K}, \mathbb{Q}_{p^{h}}^{G_{L}}\right)$ is killed by the multiplication by $[L: K]$ (using the corestriction) which induces an isomorphism on the coefficients, we have $H^{1}\left(G_{L / K}, \mathbb{Q}_{p^{h}}^{G_{L}}\right)=0$. By the inflation-restriction sequence, we obtain the assertion. Consider the case $a>0$. We denote by $\chi: G_{K} \rightarrow \mathbb{Z}_{p}^{\times}$the cyclotomic character. Then, we obtain the assertion by the following commutative diagram: 


$$
\begin{gathered}
H^{1}\left(G_{K}, \mathbb{U}_{h, a}^{\prime}\right) \stackrel{\Pi\left(N^{k} \circ \varphi^{-n}\right)_{*}}{\longrightarrow} \prod_{n, k \in \mathbb{N}} H^{1}\left(G_{K}, \mathbb{B}_{\mathrm{dR}, \mathbb{C}_{p} / K}^{+}\right) \cong \prod_{n, k \in \mathbb{N}} K \log \chi \\
\downarrow_{\operatorname{Res}_{K}^{L}} \\
H^{1}\left(G_{L}, \mathbb{U}_{h, a}^{\prime}\right) \stackrel{\downarrow^{\Pi \operatorname{Res}_{K}^{L}}}{\stackrel{\Pi\left(N^{k} \circ \varphi^{-n}\right)_{*}}{\longrightarrow}} \prod_{n, k \in \mathbb{N}} H^{1}\left(G_{L}, \mathbb{B}_{\mathrm{dR}, \mathbb{C}_{p} / K}^{+}\right) \cong \prod_{n, k \in \mathbb{N}} L \log \chi,
\end{gathered}
$$

where two isomorphisms follow by dévissage and Lemma 1.14, Theorem 1.15 (a theorem of J. Tate) and the injectivity of the horizontal arrows follow from [Colmez 2008, Proposition 0.4(ii)].

Lemma 6.2. We have

$$
\begin{gathered}
\left(\tilde{\mathbb{B}}_{\text {rig, }, \mathbb{C}_{p} / K_{0}}^{\nabla+}\right)^{\varphi^{h}=p^{-a}}=\left(\tilde{\mathbb{B}}_{\log , \mathbb{C}_{p} / K_{0}}^{\nabla+}\right)^{\varphi^{h}=p^{-a}}=0 \text { for } a \in \mathbb{N}_{>0}, \\
\left(\tilde{\mathbb{B}}_{\text {rig }, \mathbb{C}_{p} / K_{0}}^{\nabla+}\right)^{\varphi^{h}=1}=\left(\tilde{\mathbb{B}}_{\log , \mathbb{C}_{p} / K_{0}}^{\nabla+}\right)^{\varphi^{h}=1}=\mathbb{Q}_{p^{h}} .
\end{gathered}
$$

Proof. We first prove the first assertion. Suppose that we have a nonzero element $x$ in $\left(\tilde{\mathbb{B}}_{\log , \mathbb{C}_{p} / K_{0}}^{\nabla+}\right)^{\varphi^{h}=p^{-a}}$. Since $\tilde{\mathbb{B}}_{\log , \mathbb{C}_{p} / K_{0}}^{\nabla+}$ is an integral domain, we may assume that we have $x \in \mathbb{A}_{s t}, \mathbb{C}_{p} / K_{0}$ by multiplying by some power of $p$. By assumption and the $\varphi$-stability of $\mathbb{A}_{\mathrm{st},}, \mathbb{C}_{p} / K_{0}, x=p^{n a} \varphi^{n h}(x) \in p^{n} \mathbb{A}_{\mathrm{st}, \mathbb{C}_{p} / K_{0}}$. Hence $x \in \bigcap_{n} p^{n} \mathbb{A}_{\text {cris }, \mathbb{C}_{p} / K_{0}}[\mathrm{x}]=\{0\}$ since $\mathbb{A}_{\text {cris }, \mathbb{C}_{p} / K_{0}}$ is $p$-adically separated. Thus $x=0$, which is a contradiction.

We prove the latter assertion. By a simple calculation, we have

$$
\left(\tilde{\mathbb{B}}_{\log , \mathbb{C}_{p} / K_{0}}^{\nabla+}\right)^{\varphi^{h}=1}=\left(\tilde{\mathbb{B}}_{\text {rig, }, \mathbb{C}_{p} / K_{0}}^{\nabla+}\right)^{\varphi^{h}=1} .
$$

By the canonical isomorphism $\tilde{\mathbb{B}}_{\text {rig, } \mathbb{C}_{p} / K_{0}}^{\nabla+} \cong \tilde{\mathbb{B}}_{\text {rig, }}^{+}, \mathbb{C}_{p} / K_{0}^{\mathrm{pf}}$, we may reduce to the perfect residue field case, which follows from [Colmez 2002, Proposition 9.2].

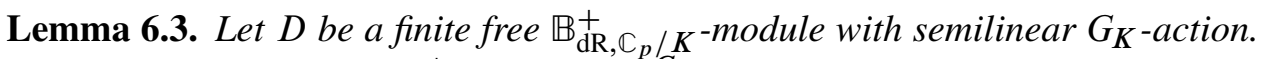
Then, the canonical map $\mathbb{B}_{\mathrm{dR}, \mathbb{C}_{p} / K}^{+} \otimes_{K} D^{G_{K}} \rightarrow D$ is injective. In particular, we have $\operatorname{dim}_{K} D^{G_{K}} \leq \operatorname{rank}_{\mathbb{B}_{\mathrm{dR}, \mathbb{C}_{p} / K}^{+}} D<\infty$.

Proof. Suppose that we have linearly independent elements $f_{1}, \ldots, f_{n} \in D^{G_{K}}$ over $K$, which have a nontrivial relation $\sum_{i} \lambda_{i} f_{i}=0$ with $\lambda_{i} \in \mathbb{B}_{\mathrm{dR}, \mathbb{C}_{p} / K}^{+}$. Choose the minimum $n$ among such $n$ 's. Then for $1 \leq i \leq n$, we have $g\left(\lambda_{i} / \lambda_{1}\right)=\lambda_{i} / \lambda_{1}$ in $\operatorname{Frac}\left(\mathbb{B}_{\mathrm{dR}, \mathbb{C}_{p} / K}\right)$. Hence we have both $\lambda_{i} / \lambda_{1} \in H^{0}\left(G_{K}, \operatorname{Frac}\left(\mathbb{B}_{\mathrm{dR}, \mathbb{C}_{p} / K}\right)\right)=K$ and $\sum_{i}\left(\lambda_{i} / \lambda_{1}\right) f_{i}=0$, a contradiction.

Lemma 6.4. Let $W$ be an $r$-dimensional $\mathbb{Q}_{p^{h}}$-vector space with semilinear $G_{K}$ -

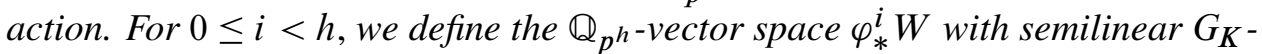
action by $\varphi_{*}^{i} W:=W$ as $G_{K}$-module with scalar multiplication

$$
\mathbb{Q}_{p^{h}} \times W \rightarrow W ; \quad(\lambda, x) \mapsto \varphi^{i}(\lambda) x .
$$


If we have an isomorphism of $\mathbb{B}_{\mathrm{dR}, \mathbb{C}_{p} / K}^{+}\left[G_{K}\right]$-modules

$$
\mathbb{B}_{\mathrm{dR}, \mathbb{C}_{p} / K}^{+} \otimes_{\mathbb{Q}_{p} h} \varphi_{*}^{i} W \cong\left(\mathbb{B}_{\mathrm{dR}, \mathbb{C}_{p} / K}^{+}\right)^{r}
$$

for $0 \leq i<h$, then $W$ is $\mathbb{C}_{p}$-admissible as a p-adic representation of $G_{K}$.

Proof. By assumption, we have isomorphisms

$$
\mathbb{B}_{\mathrm{dR}, \mathbb{C}_{p} / K}^{+} \otimes_{\mathbb{Q}_{p}} W \cong \bigoplus_{0 \leq i<h} \mathbb{B}_{\mathrm{dR}, \mathbb{C}_{p} / K}^{+} \otimes_{\mathbb{Q}_{p} h} \varphi_{*}^{i} W \cong\left(\mathbb{B}_{\mathrm{dR}, \mathbb{C}_{p} / K}^{+}\right)^{h r}
$$

of $\mathbb{B}_{\mathrm{dR}, \mathbb{C}_{p} / K}^{+}\left[G_{K}\right]$-modules, which implies the assertion by tensoring with $\mathbb{C}_{p}$ over $\mathbb{B}_{\mathrm{dR}, \mathbb{C}_{p} / K}^{+}$.

Lemma 6.5. Assume that $e_{K / K_{\text {can }}}=1$. Then, the complex $K \otimes_{K_{0}}\left(\mathbb{B}_{\text {cris }, \mathbb{C}_{p} / K_{0}}^{+}\right)^{G_{K^{\mathrm{pf}}}} \stackrel{\nabla}{\rightarrow}$

$$
\Omega_{K}^{1} \hat{\otimes}_{K_{0}}\left(\mathbb{B}_{\text {cris }, \mathbb{C}_{p} / K_{0}}^{+}\right)^{G_{K} \mathrm{pf}^{\nabla_{1}}} \Omega_{K}^{2} \hat{\otimes}_{K_{0}}\left(\mathbb{B}_{\text {cris }, \mathbb{C}_{p} / K_{0}}^{+}\right)^{G_{K \mathrm{pf}}},
$$

which is induced by the inclusion $K \otimes_{K_{0}} \mathbb{B}_{\text {cris, } \mathbb{C}_{p} / K_{0}}^{+} \rightarrow \mathbb{B}_{\mathrm{dR}, \mathbb{C}_{p} / K}^{+}$(Proposition 3.16) and Lemma 3.9, is exact. Here, we endow $\left(\mathbb{B}_{\text {cris, }}^{+} \mathbb{C}_{p} / K_{0}\right){ }^{G_{K^{\mathrm{pf}}}}$ with the p-adic topology induced by the $p$-adic semivaluation $v_{\text {cris, }, \mathbb{C}_{p} / K}$.

Proof. Note that the connections are $K_{\text {can }}$-linear by Proposition 1.13. We may reduce to the case $K=K_{0}$ by Remark 1.4(ii) and Lemma 1.10(iii). Let $\omega \in \operatorname{ker} \nabla_{1}$. We can write $\omega=\sum_{j \in J_{K}} d t_{j} \otimes \lambda_{j}$ with $\lambda_{j} \in \mathbb{B}_{\text {cris, } \mathbb{C}_{p} / K}^{+}$such that

$$
\left\{v_{\text {cris }, \mathbb{C}_{p} / K}\left(\lambda_{j}\right)\right\}_{j \in J_{K}} \rightarrow \infty \text {. }
$$

We can also write $\lambda_{j}=\sum_{\boldsymbol{n} \in \mathbb{N} \oplus J_{K}} \lambda_{j, \boldsymbol{n}} \boldsymbol{u}^{[\boldsymbol{n}]}$ with $\lambda_{j, \boldsymbol{n}} \in \mathbb{B}_{\text {cris, } \mathbb{C}_{p} / \mathbb{Q}_{p}}^{+}$such that $\left\{v_{\text {cris, } \mathbb{C}_{p} / \mathbb{Q}_{p}}\left(\lambda_{j, \boldsymbol{n}}\right)\right\}_{\boldsymbol{n} \in \mathbb{N} \oplus J_{K}} \rightarrow \infty$. Since $u_{j}$ is invariant under the action of $G_{K^{\text {pf }}}$,

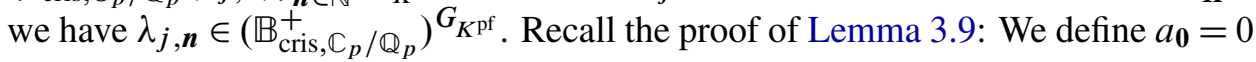
and $a_{\boldsymbol{n}}=\lambda_{j, \boldsymbol{n}-\mathbf{e}_{j}}$ if $n_{j} \neq 0$. Then, we have

$$
x=\sum_{\boldsymbol{n} \in \mathbb{N} \oplus J_{K}} a_{\boldsymbol{n}} \boldsymbol{u}^{[\boldsymbol{n}]} \in \mathbb{B}_{\mathrm{dR}, \mathbb{C}_{p} / K}
$$

and $\nabla(x)=\omega$. Note that we have $x \in\left(\mathbb{B}_{\mathrm{dR}, \mathbb{C}_{p} / K}^{+}\right)^{G_{K^{\mathrm{pf}}}}$. Hence, we have only to prove $x \in \mathbb{B}_{\text {cris, } \mathbb{C}_{p} / K}^{+}$. Fix $N \in \mathbb{N}$ : we have to show that $v_{\text {cris, } \mathbb{C}_{p} / K}\left(a_{n}\right) \geq N$ for all but finitely many $n \in \mathbb{N} \oplus J_{K}$. Choose a finite subset $J$ of $J_{K}$ such that we have $v_{\text {cris, } \mathbb{C}_{p} / K}\left(\lambda_{j}\right) \geq N$ for $j \in J_{K} \backslash J$. We also choose $n \in \mathbb{N}$ such that we have $v_{\text {cris, } \mathbb{C}_{p} / \mathbb{Q}_{p}}\left(\lambda_{j, \boldsymbol{n}}\right) \geq N$ for $j \in J$ and $|\boldsymbol{n}| \geq n$. Let $\boldsymbol{n} \in \mathbb{N} \oplus J_{K} \backslash \mathbb{N}^{J}$. Then, we have $v_{\text {cris, }, \mathbb{C}_{p} / \mathbb{Q}_{p}}\left(a_{\boldsymbol{n}}\right)=v_{\text {cris, } \mathbb{C}_{p} / \mathbb{Q}_{p}}\left(\lambda_{j, \boldsymbol{n}-\boldsymbol{e}_{j}}\right) \geq N$ for some $j \in J_{K} \backslash J$.

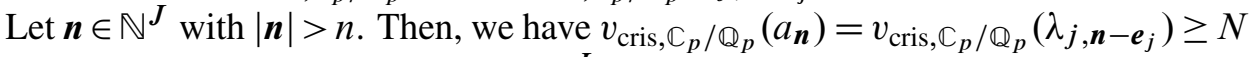
for some $j \in J$. Since the set $\left\{\boldsymbol{n} \in \mathbb{N}^{J}|| \boldsymbol{n} \mid \leq n\right\}$ is finite, these inequalities imply the assertion. 
Proof of Main Theorem. Obviously, we may assume $r:=\operatorname{dim}_{\mathbb{Q}_{p}} V>0$. By Hilbert 90 , we may replace $K$ by $K^{\mathrm{ur}}$. Hence, we may assume that $k_{K}$ is separably closed. After some Tate twist, we may also assume that $V$ satisfies the assumption of Section 5, that is, we have $\mathbb{D}_{\mathrm{dR}}(V)=\left(\mathbb{B}_{\mathrm{dR}, \mathbb{C}_{p} / K}^{+} \otimes_{\mathbb{Q}_{p}} V\right)^{G_{K}}$.

We divide the rest of the proof into two steps: We will construct a finite extension $L / K$ in Step 1 and after replacing $K$ by $L$, we will prove the semistability of $V$ in Step 2. Note that only Step 2 involves the choice of $K_{0}$.

Step 1: Set $\mu:=\widetilde{\mathbb{N}}_{\text {rig }}^{\nabla+}(V)$ and let $e_{1}, \ldots, e_{r}$ be as in Proposition 5.3. Also let $\left\{a_{1}^{\prime}<\cdots<a_{r^{\prime}}^{\prime}\right\}$ be the set of distinct elements in the multiset $\left\{a_{1}, \ldots, a_{r}\right\}$ and $m_{i}$ the multiplicity of $a_{i}^{\prime}$ in the multiset for $1 \leq i \leq r^{\prime}$. Let $\left\{e_{1}^{(i)}, \ldots, e_{m_{i}}^{(i)}\right\}$ be the subset of $e_{l} \in\left\{e_{1}, \ldots, e_{r}\right\}$ satisfying $\varphi^{h}\left(e_{l}\right)=p^{a_{i}^{\prime}} e_{l}$. We define an exhaustive and separated increasing filtration of $\mathcal{M}$ by

$$
\mu_{n}:= \begin{cases}0 & \text { if } n \leq 0, \\ \bigoplus_{1 \leq i \leq n}\left(\widetilde{\mathbb{B}}_{\mathrm{rig}, \mathbb{C}_{p} / K_{0}}^{\nabla+} e_{1}^{(i)} \oplus \cdots \oplus \widetilde{\mathbb{B}}_{\mathrm{rig}, \mathbb{C}_{p} / K_{0}}^{\nabla+} e_{m_{i}}^{(i)}\right) & \text { if } 1 \leq n<r^{\prime}, \\ & \text { otherwise. }\end{cases}
$$

The filtration is stable under $\varphi$ and $G_{K}$-actions. In fact, for $1 \leq i \leq n<r^{\prime}$ and $g \in G_{K}$, we have

$$
\varphi\left(e_{1}^{(i)}\right), \ldots, \varphi\left(e_{m_{i}}^{(i)}\right), g\left(e_{1}^{(i)}\right), \ldots, g\left(e_{m_{i}}^{(i)}\right) \in \mathcal{M}^{\varphi^{h}=p^{a_{i}^{\prime}}} \subset \mathcal{M}_{n},
$$

where the last inclusion follows from Lemma 6.2. We also define

$$
W_{n}:=\left(M_{n} / M_{n-1}\right)^{\varphi^{h}}=p^{a_{n}^{\prime}}
$$

for $1 \leq n \leq r^{\prime}$. Since we have $W_{n}=\mathbb{Q}_{p^{h}} \bar{e}_{1}^{(n)} \oplus \cdots \oplus \mathbb{Q}_{p^{h}} \bar{e}_{m_{n}}^{(n)}$ by Lemma 6.2 (where $\bar{e}_{i}^{(n)}$ denotes the image of $e_{i}^{(n)}$ in $\left.\mathcal{M}_{n} / \mathcal{M}_{n-1}\right), W_{n}$ is an $m_{n}$-dimensional $\mathbb{Q}_{p^{h}}$-vector space with continuous semilinear $G_{K}$-action. Let

$$
D_{n}:=\mathbb{B}_{\mathrm{dR}, \mathbb{C}_{p} / K}^{+} \otimes_{\widetilde{\mathbb{B}}_{\mathrm{rig}, \mathbb{C}_{p} / K_{0}}^{+}} M_{n} .
$$

Then, we have the left exact sequence of finite $K$-vector spaces

$$
0 \longrightarrow D_{n-1}^{G_{K}} \stackrel{\text { inc. }}{\longrightarrow} D_{n}^{G_{K}} \stackrel{\text { pr. }}{\longrightarrow}\left(D_{n} / D_{n-1}\right)^{G_{K}} .
$$

Hence, we have the inequalities

$$
\operatorname{dim}_{K} D_{n}^{G_{K}} \leq \operatorname{dim}_{K} D_{n-1}^{G_{K}}+\operatorname{dim}_{K}\left(D_{n} / D_{n-1}\right)^{G_{K}} \leq \operatorname{dim}_{K} D_{n-1}^{G_{K}}+m_{n}
$$

for $n \in \mathbb{Z}$ by Lemma 6.3. By Proposition 5.4, we have an isomorphism of $\mathbb{B}_{\mathrm{dR}}^{+}\left[G_{K}\right]$ modules

$$
\mathbb{B}_{\mathrm{dR}, \mathbb{C}_{p} / K}^{+} \otimes_{\widetilde{\mathbb{B}}_{\mathrm{rig}, \mathbb{C}_{p} / K_{0}}}^{+} M \cong\left(\mathbb{B}_{\mathrm{dR}, \mathbb{C}_{p} / K}^{+}\right)^{r},
$$

which implies $\operatorname{dim}_{K} D_{n}^{G_{K}}=r$ for $n \geq r^{\prime}$. Hence, the summation of the above inequalities are equalities. Therefore, the above inequalities are equalities, in 
particular, the map pr. : $D_{n}^{G_{K}} \rightarrow\left(D_{n} / D_{n-1}\right)^{G_{K}}$ in (3) is surjective. Thus, we have the commutative diagram

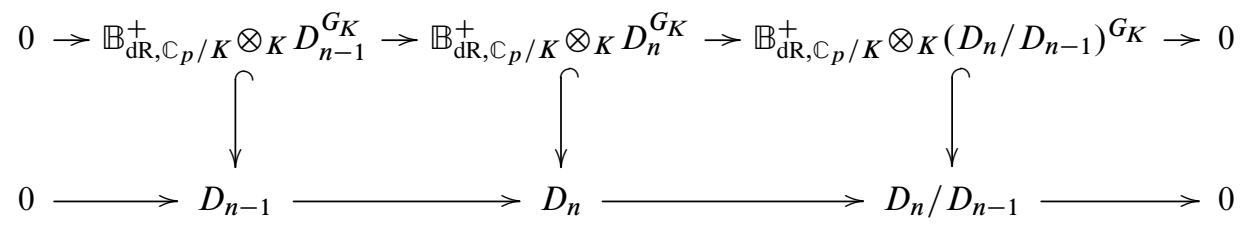

with exact rows and injective vertical arrows by Lemma 6.3. Since the middle vertical arrow is an isomorphism for $n \geq r^{\prime}$ by (4), all vertical arrows are isomorphisms. In particular, for $1 \leq n \leq r^{\prime}$, we have isomorphisms of $\mathbb{B}_{\mathrm{dR}, \mathbb{C}_{p} / K}^{+}\left[G_{K}\right]$ modules $\mathbb{B}_{\mathrm{dR}, \mathbb{C}_{p} / K}^{+} \otimes_{\mathbb{Q}_{p} h} W_{n} \cong D_{n} / D_{n-1} \cong\left(\mathbb{B}_{\mathrm{dR}, \mathbb{C}_{p} / K}^{+}\right)^{m_{n}}$. Since $W_{n}$ is stable under the action of $\varphi$, the map $W_{n} \rightarrow \varphi_{*}^{i} W_{n}$ taking $x$ to $\varphi^{i}(x)$ is an isomorphism of $\mathbb{Q}_{p^{h}}\left[G_{K}\right]$-modules. In particular, we have isomorphisms of $\mathbb{B}_{\mathrm{dR}, \mathbb{C}_{p} / K}^{+}\left[G_{K}\right]$ modules

$$
\mathbb{B}_{\mathrm{dR}, \mathbb{C}_{p} / K}^{+} \otimes_{\mathbb{Q}_{p} h} \varphi_{*}^{i} W_{n} \cong \mathbb{B}_{\mathrm{dR}, \mathbb{C}_{p} / K}^{+} \otimes_{\mathbb{Q}_{p} h} W_{n} \cong\left(\mathbb{B}_{\mathrm{dR}, \mathbb{C}_{p} / K}^{+}\right)^{m_{n}}
$$

for $1 \leq n \leq r^{\prime}$ and $0 \leq i<h$, which implies the $\mathbb{C}_{p}$-admissibility of $W_{n}$ by Lemma 6.4. Hence, $G_{K}$ acts on $W_{n}$ factoring through a finite quotient by Theorem 2.1. We choose a finite extension $L / K$ such that $G_{L}$ acts on $W_{n}$ trivially for all $1 \leq n \leq r^{\prime}$ and such that $L$ satisfies condition $(\mathrm{H})$.

Step 2: By replacing $V$ by $\left.V\right|_{L}$, we will prove that $V$ is semistable by calculating Galois cohomology associated to $\widetilde{\mathbb{N}}_{\text {rig }}^{\nabla+}(V)$. In the following, we fix $K_{0}$ and a lift $\left\{t_{j}\right\}_{j \in J_{K}}$ of a $p$-basis of $k_{K}$ in $K_{0}$. We regard $\left\{t_{j}\right\}_{j \in J_{K}}$ as a lift of a $p$-basis of $k_{K}$ in $K$. We also fix notation: For a commutative ring $R$, let $U_{r}(R) \subset \mathrm{GL}_{r}(R)$ be the group of unipotent upper triangular matrices. Let $N_{r}(R) \subset M_{r}(R)$ be the Lie algebra of $U_{r}(R)$, that is, the group of nilpotent upper triangular matrices. We denote $U_{r, \mathrm{dR}}^{+}:=U_{r}\left(\mathbb{B}_{\mathrm{dR}, \mathbb{C}_{p} / K}^{+}\right), U_{r, \mathrm{dR}}^{\nabla+}=U_{r}\left(\mathbb{B}_{\mathrm{dR}, \mathbb{C}_{p} / K}^{\nabla+}\right)$ for simplicity.

By assumption, we have $g\left(e_{1}, \ldots, e_{r}\right)=\left(e_{1}, \ldots, e_{r}\right) c_{g}$ with 1-cocycle

$$
c: G_{K} \rightarrow U_{r}\left(\widetilde{\mathbb{B}}_{\text {rig, }}^{\nabla+} \mathbb{C}_{p} / K_{0}\right) \text {. }
$$

Since we have $\widetilde{\mathbb{N}}_{\text {rig }}^{\nabla+}(V) \subset \widetilde{\mathbb{B}}_{\text {rig, }}^{\nabla+} \mathbb{C}_{p} / K_{0} \otimes_{\mathbb{Q}_{p}} V$ and

$$
\left(K \otimes_{K_{0}} \mathbb{B}_{\mathrm{st}, \mathbb{C}_{p} / K_{0}}^{+} \otimes_{\mathbb{Q}_{p}} V\right)^{G_{K}}=K \otimes_{K_{0}}\left(\mathbb{B}_{\mathrm{st}, \mathbb{C}_{p} / K_{0}}^{+} \otimes_{\mathbb{Q}_{p}} V\right)^{G_{K}},
$$

we have only to prove that $c$ is a 1-coboundary in $U_{r}\left(K \otimes_{K_{0}} \mathbb{B}_{\mathrm{st}, \mathbb{C}_{p} / K_{0}}^{+}\right)$. We have the exact sequence of pointed sets

$$
\left(U_{r, \mathrm{dR}}^{+} / U_{r, \mathrm{dR}}^{\nabla+}\right)^{G_{K}} \stackrel{\delta}{\longrightarrow} H^{1}\left(G_{K}, U_{r, \mathrm{dR}}^{\nabla+}\right) \stackrel{\text { inc.* }}{\longrightarrow} H^{1}\left(G_{K}, U_{r, \mathrm{dR}}^{+}\right),
$$

where $U_{r, \mathrm{dR}}^{+} / U_{r, \mathrm{dR}}^{\nabla+}$ denotes the left coset of $U_{r, \mathrm{dR}}^{+}$by $U_{r, \mathrm{dR}}^{\nabla+}$, that is, $X \sim Y$ if $X^{-1} Y \in U_{r, \mathrm{dR}}^{\nabla+}$. The class $[c] \in H^{1}\left(G_{K}, U_{r, \mathrm{dR}}^{\nabla+}\right)$ represented by $c$ is mapped to 
the trivial class in $H^{1}\left(G_{K}, U_{r, \mathrm{dR}}^{+}\right)$. In fact, since we have $\bar{e}_{i}^{(n)} \in\left(D_{n} / D_{n-1}\right)^{G_{K}}$ for $1 \leq n \leq r^{\prime}$ and $1 \leq i \leq m_{n}$ by assumption, there exists an element $\tilde{e}_{i}^{(n)} \in D_{n}^{G_{K}}$ such that $\tilde{e}_{i}^{(n)}-e_{i}^{(n)} \in D_{n-1}$ by the exactness of (3). Then,

$$
\left(\tilde{e}_{1}^{(1)}, \ldots, \tilde{e}_{m_{1}}^{(1)}, \ldots, \tilde{e}_{1}^{(n)}, \ldots, \tilde{e}_{m_{n}}^{(n)}\right)
$$

is a $\mathbb{B}_{\mathrm{dR}, \mathbb{C}_{p} / K^{-}}^{+}$basis of $D_{n}$ for $1 \leq n \leq r^{\prime}$ and we have a unique matrix $U \in U_{r, \mathrm{dR}}^{+}$ such that

$$
\left(e_{1}^{(1)}, \ldots, e_{m_{1}}^{(1)}, \ldots, e_{1}^{\left(r^{\prime}\right)}, \ldots, e_{m_{r^{\prime}}}^{\left(r^{\prime}\right)}\right)=\left(\tilde{e}_{1}^{(1)}, \ldots, \tilde{e}_{m_{1}}^{(1)}, \ldots, \tilde{e}_{1}^{\left(r^{\prime}\right)}, \ldots, \tilde{e}_{m_{r^{\prime}}}^{\left(r^{\prime}\right)}\right) U
$$

By a simple calculation, we have $c_{g}=U^{-1} g(U)$ for all $g \in G_{K}$. Hence, the class $[c]$ is represented by an element of the image of $\left(U_{r, \mathrm{dR}}^{+} / U_{r, \mathrm{dR}}^{\nabla+}\right)^{G_{K}}$ under $\delta$ by the exact sequence (5). We regard $K \otimes_{K_{0}} \mathbb{B}_{\text {cris, } \mathbb{C}_{p} / K_{0}}^{+}$as a subring of $\mathbb{B}_{\mathrm{dR}, \mathbb{C}_{p} / K}^{+}$by Proposition 3.16. Then, we have the following lemma:

Lemma 6.6. Every element of $\left(U_{r, \mathrm{dR}}^{+} / U_{r, \mathrm{dR}}^{\nabla+}\right)^{G_{K}}$ is represented by an element in $U_{r}\left(K \otimes_{K_{0}}\left(\mathbb{B}_{\text {cris, } \mathbb{C}_{p} / K_{0}}^{+}\right)^{G_{K \mathrm{pf}}}\right)$.

We leave the proof of Lemma 6.6 to the end of the proof. Thanks to the lemma, there exist $X_{1} \in U_{r}\left(K \otimes_{K_{0}}\left(\mathbb{B}_{\text {cris, } \mathbb{C}_{p} / K_{0}}\right)^{G_{K^{\mathrm{pf}}}}\right)$ and $X_{2} \in U_{r, \mathrm{dR}}^{\nabla+}$ such that

$$
c_{g}=X_{2}^{-1} X_{1}^{-1} g\left(X_{1}\right) g\left(X_{2}\right)
$$

for all $g \in G_{K}$.

Since the canonical isomorphism $i: \tilde{\mathbb{B}}_{\mathrm{rig}, \mathbb{C}_{p} / K_{0}}^{\nabla+} \rightarrow \tilde{\mathbb{B}}_{\mathrm{rig}, \mathbb{C}_{p} / K_{0}^{\mathrm{pf}}}^{+}$is compatible with the actions of $\varphi$ and $G_{K^{\text {pf }}}$, we may regard $M:=i^{*} \mathcal{M}$ as a $\left(\varphi, G_{K^{\text {pf }}}\right)$-module over $\tilde{\mathbb{B}}_{\mathrm{rig},}^{+}, \mathbb{C}_{p} / K_{0}^{\mathrm{pf}}$. Then, the triple $\left(M,\left\{e_{1}, \ldots, e_{r}\right\}, i^{*} c\right)$ satisfies the assumptions of Proposition 6.1. Indeed, assumption (i) follows from Proposition 5.3, Proposition 5.4 and the functoriality. The image of $c$ is in $U_{r}\left(\tilde{\mathbb{B}}_{\text {rig, } \mathbb{C}_{p} / K_{0}}^{\nabla+}\right)$, which implies assumption (ii). Applying Proposition 6.1 to the above triple, we have $X_{3}^{\prime} \in U_{r}\left(\mathbb{B}_{\mathrm{st}, \mathbb{C}_{p} / K_{0}^{\mathrm{pf}}}^{+}\right)$ such that $i\left(c_{g}\right)=\left(X_{3}^{\prime}\right)^{-1} g\left(X_{3}^{\prime}\right)$. Hence, $X_{3}:=i^{-1}\left(X_{3}^{\prime}\right) \in U_{r}\left(\mathbb{B}_{\mathrm{st}, \mathbb{C}_{p} / K_{0}}\right)$ satisfies $c_{g}=X_{3}^{-1} g\left(X_{3}\right)$ for $g \in G_{K^{\mathrm{pf}}}$. Since we have $c_{g}=X_{2}^{-1} g\left(X_{2}\right)$ for $g \in G_{K^{\mathrm{pf}}}$ by (6), we have

$$
X_{2} X_{3}^{-1} \in\left(U_{r, \mathrm{dR}}^{\nabla+}\right)^{G_{K \mathrm{pf}}}=U_{r}\left(\left(\mathbb{B}_{\mathrm{dR}, \mathbb{C}_{p} / K}^{\nabla+}\right)^{G_{K \mathrm{pf}}}\right) .
$$

Note that the canonical map

$$
K_{\text {can }} \otimes_{K_{\text {can }, 0}}\left(\mathbb{B}_{\text {cris, } \mathbb{C}_{p} / K_{0}}^{\nabla+}\right)^{G_{K \mathrm{pf}}} \rightarrow\left(\mathbb{B}_{\mathrm{dR}, \mathbb{C}_{p} / K}^{\nabla+}\right)^{G_{K^{\mathrm{pf}}}}
$$

is an isomorphism. In fact, by using the canonical isomorphisms $\mathbb{B}_{\text {cris, } \mathbb{C}_{p} / K_{0}}^{\nabla+} \rightarrow$

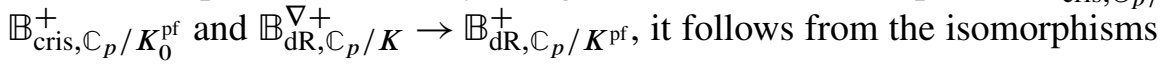

$$
K_{\mathrm{can}} \otimes_{K_{\mathrm{can}, 0}} K_{0}^{\mathrm{pf}} \cong K \otimes_{K_{0}} K_{0}^{\mathrm{pf}} \cong K^{\mathrm{pf}}
$$


where the first isomorphism easily follows from Remark 1.4(ii) and the second one is trivial. Thus, we have

$$
c_{g}=\left(X_{1} \cdot X_{2} X_{3}^{-1} \cdot X_{3}\right)^{-1} g\left(X_{1} \cdot X_{2} X_{3}^{-1} \cdot X_{3}\right)
$$

for all $g \in G_{K}$ with $X_{1}, X_{2} X_{3}^{-1}, X_{3} \in U_{r}\left(K \otimes_{K_{0}} \mathbb{B}_{\text {st, } \mathbb{C}_{p} / K_{0}}^{+}\right)$, which implies the assertion.

Now, we return to the proof of Lemma 6.6. We endow $M_{r}\left(\mathbb{B}_{\mathrm{dR}, \mathbb{C}_{p} / K}^{+}\right) \cong$ $\left(\mathbb{B}_{\mathrm{dR}, \mathbb{C}_{p} / K}^{+}\right)^{r^{2}}$ with the product topology. Let

$$
\begin{aligned}
& d: M_{r}\left(\mathbb{B}_{\mathrm{dR}, \mathbb{C}_{p} / K}^{+}\right) \rightarrow \hat{\Omega}_{K}^{1} \hat{\otimes}_{K} M_{r}\left(\mathbb{B}_{\mathrm{dR}, \mathbb{C}_{p} / K}^{+}\right) ; \quad\left(x_{i j}\right) \mapsto\left(\nabla\left(x_{i j}\right)\right), \\
& d_{1}: \hat{\Omega}_{K}^{1} \hat{\otimes}_{K} M_{r}\left(\mathbb{B}_{\mathrm{dR}, \mathbb{C}_{p} / K}^{+}\right) \rightarrow \hat{\Omega}_{K}^{2} \hat{\otimes}_{K} M_{r}\left(\mathbb{B}_{\mathrm{dR}, \mathbb{C}_{p} / K}^{+}\right) ; \quad\left(\omega_{i j}\right) \mapsto\left(\nabla_{1}\left(\omega_{i j}\right)\right)
\end{aligned}
$$

be the derivations. For $i \in\{1,2\}$, we endow $\widehat{\Omega}_{K}^{i} \hat{\otimes}_{K} M_{r}\left(\mathbb{B}_{\mathrm{dR}, \mathbb{C}_{p} / K}^{+}\right)$with the left (resp. right) action of $M_{r}\left(\mathbb{B}_{\mathrm{dR}, \mathbb{C}_{p} / K}^{+}\right)$induced by the left (resp. right) multiplication on $M_{r}\left(\mathbb{B}_{\mathrm{dR}, \mathbb{C}_{p} / K}^{+}\right)$. We also have the wedge product

$$
\begin{gathered}
\wedge: \hat{\Omega}_{K}^{1} \hat{\otimes}_{K} N_{r}(K) \times \hat{\Omega}_{K}^{1} \hat{\otimes}_{K} N_{r}\left(\mathbb{B}_{\mathrm{dR}, \mathbb{C}_{p} / K}^{+}\right) \rightarrow \hat{\Omega}_{K}^{2} \hat{\otimes}_{K} N_{r}\left(\mathbb{B}_{\mathrm{dR}, \mathbb{C}_{p} / K}^{+}\right) \\
\left(\omega_{i j}\right) \times\left(\omega_{i j}^{\prime}\right) \mapsto\left(\sum_{1 \leq k \leq r} \omega_{i k} \wedge \omega_{k j}^{\prime}\right) .
\end{gathered}
$$

Then, we have the formulas $d_{1} \circ d=0, d\left(X X^{\prime}\right)=d X \cdot X^{\prime}+X \cdot d X^{\prime}, d_{1}(\omega \cdot X)=$ $d_{1} \omega \cdot X-\omega \wedge d X$, and $\left(\omega \wedge \omega^{\prime}\right) \cdot X=\omega \wedge\left(\omega^{\prime} \cdot X\right)$, for $X, X^{\prime} \in \widehat{\Omega}_{K}^{1} \hat{\otimes}_{K} N_{r}\left(\mathbb{B}_{\mathrm{dR}, \mathbb{C}_{p} / K}^{+}\right)$, $\omega \in \widehat{\Omega}_{K}^{1} \hat{\otimes}_{K} N_{r}(K)$, and $\omega^{\prime} \in \widehat{\Omega}_{K}^{1} \hat{\otimes}_{K} N_{r}\left(\mathbb{B}_{\mathrm{dR}, \mathbb{C}_{p} / K}^{+}\right)$. We define a log differential

$$
\operatorname{dlog}: U_{r, \mathrm{dR}}^{+} \rightarrow \hat{\Omega}_{K}^{1} \hat{\otimes}_{K} N_{r}\left(\mathbb{B}_{\mathrm{dR}, \mathbb{C}_{p} / K}^{+}\right) ; \quad X \mapsto d X \cdot X^{-1},
$$

which is $G_{K}$-equivariant. (Note that it does not preserve the group laws in general.) Since we have $\operatorname{dlog}(X A)=\operatorname{d} \log (X)$ for $A \in U_{r, \mathrm{dR}}^{\nabla+}$ and $X \in U_{r, \mathrm{dR}}^{+}$by the above formulas, dlog induces a morphism of $G_{K}$-sets

$$
\operatorname{dlog}_{*}: U_{r, \mathrm{dR}}^{+} / U_{r, \mathrm{dR}}^{\nabla+} \rightarrow \hat{\Omega}_{K}^{1} \hat{\otimes}_{K} N_{r}\left(\mathbb{B}_{\mathrm{dR}, \mathbb{C}_{p} / K}^{+}\right) .
$$

Moreover, $\operatorname{dlog}_{*}$ is injective. Indeed, let $X, Y \in U_{r, \mathrm{dR}}^{+}$such that $\operatorname{dlog} X=\mathrm{d} \log Y$. By $d \boldsymbol{E}=d\left(Y^{-1} Y\right)=0$ and the above formulas, we have $d\left(Y^{-1}\right)=-Y^{-1} d Y \cdot Y^{-1}$. Hence, we have

$$
\begin{aligned}
\operatorname{dlog}\left(Y^{-1} X\right) & =\left(d\left(Y^{-1}\right) \cdot X+Y^{-1} d X\right) \cdot X^{-1} Y \\
& =-Y^{-1}\left(d Y \cdot Y^{-1}-d X \cdot X^{-1}\right) \cdot Y=0 .
\end{aligned}
$$

Since the inverse image of $\{0\}$ by dlog is $U_{r, \mathrm{dR}}^{\nabla+}$, we have $X \sim Y$. By taking $H^{0}\left(G_{K},-\right)$ of $\operatorname{dlog}_{*}$, we have an injection of sets

$$
\operatorname{dlog}_{*}:\left(U_{r, \mathrm{dR}}^{+} / U_{r, \mathrm{dR}}^{\nabla+}\right) G_{K} \hookrightarrow \widehat{\Omega}_{K}^{1} \hat{\otimes}_{K} N_{r}(K) .
$$


We define a decreasing filtration on $N_{r}\left(\mathbb{B}_{\mathrm{dR}, \mathbb{C}_{p} / K}^{+}\right)$by

$$
\mathrm{Fil}^{n} N_{r}\left(\mathbb{B}_{\mathrm{dR}, \mathbb{C}_{p} / K}^{+}\right):=\left\{\left(a_{i j}\right) \in N_{r}\left(\mathbb{B}_{\mathrm{dR}, \mathbb{C}_{p} / K}^{+}\right) \mid a_{i j}=0 \text { if } j-i \leq n\right\} .
$$

Then, we have Fil ${ }^{0} N_{r}\left(\mathbb{B}_{\mathrm{dR}, \mathbb{C}_{p} / K}^{+}\right)=N_{r}\left(\mathbb{B}_{\mathrm{dR}, \mathbb{C}_{p} / K}^{+}\right)$and $\mathrm{Fil}^{r} N_{r}\left(\mathbb{B}_{\mathrm{dR}, \mathbb{C}_{p} / K}^{+}\right)=0$. Let $X \in U_{r, \mathrm{dR}}^{+}$such that we have $[X] \in\left(U_{r, \mathrm{dR}}^{+} / U_{r, \mathrm{dR}}^{\nabla+}\right)^{G_{K}}$. Let $\omega:=\operatorname{dlog}(X) \in$

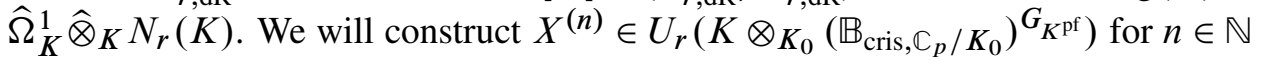
satisfying

$$
\omega \cdot X^{(n)} \equiv d X^{(n)} \quad \bmod \hat{\Omega}_{K}^{1} \hat{\otimes}_{K} \mathrm{Fil}^{n} N_{r}\left(\mathbb{B}_{\mathrm{dR}, \mathbb{C}_{p} / K}^{+}\right) .
$$

Set $X^{(0)}:=1$. Suppose that we have constructed $X^{(n)}$. Since we have $\omega \cdot X=d X$, we have $d_{1} \omega \cdot X=\omega \wedge d X$ by taking $d_{1}$. Hence, we have $d_{1} \omega=(\omega \wedge d X) \cdot X^{-1}=$ $\omega \wedge \omega$. Let $\omega^{\prime}=\left(\omega_{i j}^{\prime}\right):=\omega \cdot X^{(n)}-d X^{(n)} \in \widehat{\Omega}_{K}^{1} \widehat{\otimes}_{K} \mathrm{Fil}^{n} N_{r}\left(\mathbb{B}_{\mathrm{dR}, \mathbb{C}_{p} / K}^{+}\right)$. Then, by a simple calculation using the above formulas, we have $d_{1} \omega^{\prime}=\omega \wedge\left(\omega \cdot X^{(n)}-d X^{(n)}\right)=\omega \wedge \omega^{\prime} \equiv 0 \quad \bmod \widehat{\Omega}_{K}^{2} \hat{\otimes}_{K} \mathrm{Fil}^{n+1} N_{r}\left(\mathbb{B}_{\mathrm{dR}, \mathbb{C}_{p} / K}^{+}\right)$, which implies $\nabla_{1}\left(\omega_{i, i+n+1}^{\prime}\right)=0$. Since we have

$$
\omega_{i j}^{\prime} \in \hat{\Omega}_{K}^{1} \hat{\otimes}_{K}\left(K \otimes_{K_{0}}\left(\mathbb{B}_{\text {cris, }, \mathbb{C}_{p} / K_{0}}^{+}\right)^{G_{K^{\mathrm{pf}}}}\right),
$$

by Lemma 6.5 , there exists $x_{i, i+n+1}^{\prime} \in K \otimes_{K_{0}}\left(\mathbb{B}_{\text {cris, } \mathbb{C}_{p} / K_{0}}^{+}\right)^{G_{K^{\mathrm{pf}}}}$ such that

$$
\nabla\left(x_{i, i+n+1}^{\prime}\right)=\omega_{i, i+n+1}^{\prime} \text {. }
$$

Let $X^{(n+1)}:=X^{(n)}+\sum_{i} x_{i, i+n+1}^{\prime} E_{i, i+n+1} \in U_{r}\left(K \otimes_{K_{0}}\left(\mathbb{B}_{\text {cris }, \mathbb{C}_{p} / K_{0}}\right)^{G_{K \mathrm{pf}}}\right)$. Then, by a simple calculation, we have

$$
\begin{aligned}
\omega \cdot X^{(n+1)}-d X^{(n+1)} & \\
& \equiv \omega \cdot X^{(n)}-d X^{(n)}-d\left(\sum_{i} x_{i, i+n+1}^{\prime} E_{i, i+n+1}\right) \\
& \equiv \omega^{\prime}-\sum_{i} \nabla\left(x_{i, i+n+1}^{\prime}\right) E_{i, i+n+1} \equiv 0 \bmod \hat{\Omega}_{K} \hat{\otimes}_{K} \mathrm{Fil}^{n+1} N_{r}\left(\mathbb{B}_{\mathrm{dR}, \mathbb{C}_{p} / K}^{+}\right) .
\end{aligned}
$$

Hence, we have $\operatorname{dlog}\left(X^{(r)}\right)=\omega$, which implies the assertion.

\section{Applications}

We will give applications of the Main Theorem. In Section 7A, we will recall linear algebraic structures, which appear in the following. In Section 7B, we will prove a horizontal analogue of the $p$-adic monodromy theorem. The results of the next two subsections are applications of this theorem. In Section 7C, we will prove an equivalence between the category of horizontal de Rham representations of $G_{K}$ and the category of de Rham representation of $G_{K_{\text {can }}}$. In Section 7D, we will prove a generalization of Hyodo's Theorem 1.16. 
In this section, unless particular mention is stated, we will denote $\mathbb{B}_{\diamond, \mathbb{C}_{p} / K_{0}}^{\nabla}$ (resp. $\mathbb{B}_{\circlearrowleft, \mathbb{C}_{p} / K}^{\nabla}$ ) by $\mathbb{B}_{\diamond}^{\nabla}$ (resp. $\mathbb{B}_{\odot}^{\nabla}$ ) for $\diamond \in\{$ cris, st $\}$ (resp. $\diamond \in\{\mathrm{dR}, \mathrm{HT}\}$ ): This notation is justified by the facts that $\mathbb{B}_{\diamond, \mathbb{C}_{p} / K_{0}}^{\nabla}$ and $\mathbb{B}_{\circlearrowleft, \mathbb{C}_{p} / K}^{\nabla}$ are isomorphic to $\mathbb{B}_{\diamond, \mathbb{C}_{p} / \mathbb{Q}_{p}}$ and $\mathbb{B}_{\odot, \mathbb{C}_{p} / \mathbb{Q}_{p}}$ as $\left(\mathbb{Q}_{p}, G_{K}\right)$-rings respectively.

7A. Additional structures. In the following, let $V \in \operatorname{Rep}_{\mathbb{Q}_{p}} G_{K}$. The vector space $\mathbb{D}_{\bullet}^{\nabla}(V)$ has additional structures for $\bullet \in\{$ cris, st, $\mathrm{dR}, \mathrm{HT}\}$, which we will recall following [Fontaine 1994b].

- The Hodge-Tate case

We define a graded $K$-vector space as a finite-dimensional $K$-vector space $D$ endowed with a decomposition $D=\bigoplus_{n \in \mathbb{Z}} D_{n}$. Denote by $M G_{K}$ the category of graded $K$-vector spaces. The graded ring structure on $\mathbb{B}_{\mathrm{HT}}^{\nabla}$ induces a graded $K$ vector space structure on $\mathbb{D}_{\mathrm{HT}}^{\nabla}(V)$. Hence, we have a $\otimes$-functor

$$
\mathbb{D}_{\mathrm{HT}}^{\nabla}: \operatorname{Rep}_{\mathrm{HT}}^{\nabla} G_{K} \rightarrow M G_{K}
$$

Assume that we have $V \in \operatorname{Rep}_{\mathrm{HT}}^{\nabla} G_{K}$. We define the Hodge-Tate weights of $V$ as the multiset consisting of $n \in \mathbb{Z}$ with multiplicity $m_{n}:=\operatorname{dim}_{K}\left(\mathbb{C}_{p}(-n) \otimes_{\mathbb{Q}_{p}} V\right)^{G_{K}}$. Since the comparison isomorphism $\alpha_{\mathrm{HT}}^{\nabla}$ is compatible with $G_{K}$-actions and gradings, by taking the degree zero part, we have an isomorphism of $\mathbb{C}_{p}\left[G_{K}\right]$-modules

$$
\mathbb{C}_{p} \otimes_{\mathbb{Q}_{p}} V \cong \bigoplus_{n \in \mathbb{Z}} \mathbb{C}_{p}(n)^{m_{n}},
$$

which is referred to as the Hodge-Tate decomposition of $V$. Note that if $V \in$ $\operatorname{Rep}_{\mathbb{Q}_{p}} G_{K}$ admits such a decomposition, then it is horizontal Hodge-Tate.

- The de Rham case

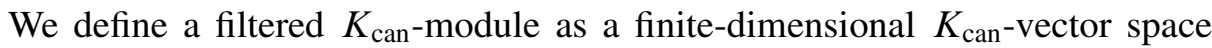
endowed with a decreasing filtration $\left\{\mathrm{Fil}^{n} D\right\}_{n \in \mathbb{Z}}$ of $K_{\text {can }}$-subspaces such that Fil $^{n} D=D$ for $n \ll 0$ and Fil ${ }^{n} D=0$ for $n \gg 0$. Denote by $M F_{K_{\text {can }}}$ the category of filtered $K_{\text {can }}$-modules. The filtration Fil $^{n} \mathbb{B}_{\mathrm{dR}}^{\nabla}=t^{n} \mathbb{B}_{\mathrm{dR}}^{\nabla+}$ on $\mathbb{B}_{\mathrm{dR}}^{\nabla}$ induces a

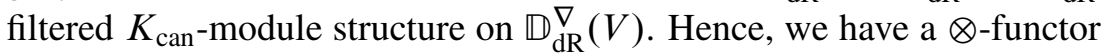

$$
\mathbb{D}_{\mathrm{dR}}^{\nabla}: \operatorname{Rep}_{\mathrm{dR}}^{\nabla} G_{K} \rightarrow M F_{K_{\mathrm{can}}} \text {. }
$$

- The crystalline and semistable cases

We first define filtered $\left(\varphi, N, G_{L / K}\right)$-modules for our later use.

Defintion 7.1. (i) Let $L / K$ be a finite Galois extension. A filtered $\left(\varphi, N, G_{L / K}\right)$ -

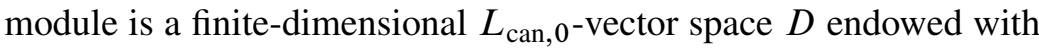

- the Frobenius endomorphism: a bijective $\varphi$-semilinear map $\varphi: D \rightarrow D$;

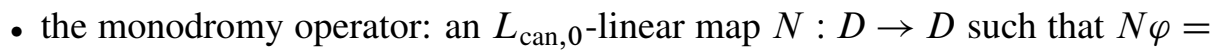
$p \varphi N$; 


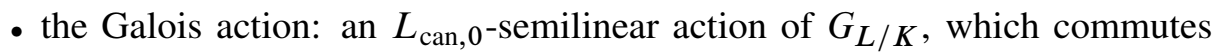
with $\varphi$ and $N$;

- the filtration: a decreasing filtration $\left\{\mathrm{Fil}^{n} D_{L_{\mathrm{can}}}\right\}_{n \in \mathbb{Z}}$ of $G_{L / K}$-stable $L_{\text {can- }}$ subspaces of $D_{L_{\text {can }}}:=L_{\text {can }} \otimes_{L_{\text {can }, 0}} D$ satisfying

$\mathrm{Fil}^{n} D_{L_{\text {can }}}=D_{L_{\text {can }}} \quad$ for $n \ll 0 \quad$ and $\quad$ Fil $^{n} D_{L_{\text {can }}}=0 \quad$ for $n \gg 0$.

If $L=K$, then we call $D$ a filtered $(\varphi, N)$-module relative to $K_{\text {can }}$. Moreover, if $N=0$, then we call $D$ a filtered $\varphi$-module relative to $K_{\text {can }}$.

A morphism $D_{1} \rightarrow D_{2}$ of filtered $\left(\varphi, N, G_{L / K}\right)$-modules is an $L_{\text {can }, 0}$-linear map $f: D_{1} \rightarrow D_{2}$ such that $f$ commutes with $\varphi$ and $N, G_{L / K}$-actions and we have $f\left(\mathrm{Fil}^{n} D_{1, L_{\text {can }}}\right) \subset \mathrm{Fil}^{n} D_{2, L_{\text {can }}}$ for all $n \in \mathbb{Z}$.

Denote by $M F\left(\varphi, N, G_{L / K}\right)$ (resp. $\left.M F_{K_{\text {can }}}(\varphi, N), M F_{K_{\text {can }}}(\varphi)\right)$ the category of filtered $\left(\varphi, N, G_{L / K}\right)$-modules (resp. filtered $(\varphi, N)$-modules relative to $K_{\text {can }}$, filtered $\varphi$-modules relative to $\left.K_{\text {can }}\right)$.

(ii) Let $D \in M F_{K_{\text {can }}}(\varphi, N)$ and $r:=\operatorname{dim}_{K_{\text {can }, 0}} D$. We define $t_{N}(D)$ and $t_{H}(D)$ in the following way: First, we consider the case $r=1$. If we have $v \in D \backslash\{0\}$ and $\varphi(v)=\lambda v$, then $v_{p}(\lambda) \in \mathbb{Z}$ is independent of the choice of $v$. We denote it by $t_{N}(D)$. We denote by $t_{H}(D)$ the maximum number $n \in \mathbb{Z}$ such that Fil $^{n} D_{K_{\text {can }}} \neq 0$. In the general case, we define

$$
t_{N}(D):=t_{N}\left(\bigwedge^{r} D\right), \quad t_{H}(D):=t_{H}\left(\bigwedge^{r} D\right) .
$$

We say that $D$ is weakly admissible if we have $t_{N}(D)=t_{H}(D)$ and $t_{N}\left(D^{\prime}\right) \geq$

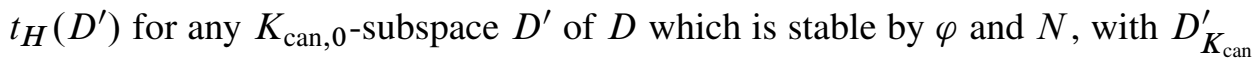
endowed with the induced filtration of $D_{K_{\text {can }}}$.

Denote by $M F^{\text {wa }}\left(\varphi, N, G_{L / K}\right)$ the full subcategory of $M F\left(\varphi, N, G_{L / K}\right)$ whose objects are weakly admissible as object of $M F_{L_{\text {can }}}(\varphi, N)$. We define $M F_{K_{\text {can }}}^{\text {wa }}(\varphi, N)$ and $M F_{K_{\text {can }}}^{\text {wa }}(\varphi)$ similarly.

Let $\diamond \in\{$ cris, st $\}$. By Proposition 3.16, we have a $K_{\text {can-linear injection }}$

$$
K_{\text {can }} \otimes_{K_{\text {can }, 0}} \mathbb{D}_{\diamond}^{\nabla}(V) \rightarrow \mathbb{D}_{\mathrm{dR}}^{\nabla}(V) .
$$

We endow $K_{\text {can }} \otimes_{K_{\text {can }, 0}} \mathbb{D}_{\diamond}^{\nabla}(V)$ with the induced filtration of $\mathbb{D}_{\mathrm{dR}}^{\nabla}(V)$. Together with the Frobenius endomorphism $\varphi$ and the monodromy operator $N$ on $\mathbb{B}_{\mathrm{st}}^{\nabla}$, these data induce a structure of a filtered $(\varphi, N)$-module over $K_{\text {can }}$ relative to $K_{\text {can, } 0}$ on $\mathbb{D}_{\diamond}^{\nabla}(V)$. Since we have $\mathbb{D}_{\text {cris }}^{\nabla}(V)=\left(\mathbb{D}_{\text {st }}^{\nabla}(V)\right)^{N=0}, \mathbb{D}_{\text {cris }}^{\nabla}(V)$ has a structure of a filtered $\varphi$-module over $K_{\text {can }}$ relative to $K_{\text {can, } 0}$. Therefore, we have $\otimes$-functors

$$
\mathbb{D}_{\text {cris }}^{\nabla}: \operatorname{Rep}_{\text {cris }}^{\nabla} G_{K} \rightarrow M F_{K_{\text {can }}}(\varphi), \quad \mathbb{D}_{\text {st }}^{\nabla}: \operatorname{Rep}_{\text {st }}^{\nabla} G_{K} \rightarrow M F_{K_{\text {can }}}(\varphi, N) .
$$

For $D \in M F_{K_{\text {can }}}(\varphi, N)$, we define

$$
\mathbb{V}_{\mathrm{st}}(D):=\left(\mathbb{B}_{\mathrm{st}}^{\nabla} \otimes_{K_{\mathrm{can}, 0}} D\right)^{N=0, \varphi=1} \cap \mathrm{Fil}^{0}\left(\mathbb{B}_{\mathrm{dR}}^{\nabla} \otimes_{K_{\mathrm{can}}} D_{K_{\text {can }}}\right) .
$$


For $D \in M F_{K_{\text {can }}}(\varphi)$, we define $\mathbb{V}_{\text {cris }}(D):=\mathbb{V}_{\text {st }}(D)$. These are (possibly infinitedimensional) $\mathbb{Q}_{p}$-vector spaces with $G_{K}$-action.

Remark 7.2. Note that we have the hierarchy of full subcategories of $\operatorname{Rep}_{\mathbb{Q}_{p}} G_{K}$

$$
\operatorname{Rep}_{\mathrm{cris}}^{\nabla} G_{K} \subset \operatorname{Rep}_{\mathrm{st}}^{\nabla} G_{K} \subset \operatorname{Rep}_{\mathrm{dR}}^{\nabla} G_{K} \subset \operatorname{Rep}_{\mathrm{HT}}^{\nabla} G_{K}
$$

In fact, if we have $V \in \operatorname{Rep}_{\text {cris }}^{\nabla} G_{K}$, then we have $\operatorname{dim}_{\mathbb{Q}_{p}} V=\operatorname{dim}_{K_{\text {can }, 0}} \mathbb{D}_{\text {cris }}^{\nabla}(V) \leq$ $\operatorname{dim}_{K_{\text {can }, 0}} \mathbb{D}_{\mathrm{st}}^{\nabla}(V)$, which implies that $V$ is horizontal semistable by Lemma 1.19. In this case, the canonical injection $\mathbb{D}_{\text {cris }}^{\nabla}(V) \hookrightarrow \mathbb{D}_{\mathrm{st}}^{\nabla}(V)$ is an isomorphism as filtered $(\varphi, N)$-modules relative to $K_{\text {can }}$. The inclusion $\operatorname{Rep}_{\mathrm{st}}^{\nabla} G_{K} \subset \operatorname{Rep}_{\mathrm{dR}}^{\nabla} G_{K}$ follows from Lemma 1.20, Proposition 3.16 and Corollary 4.3. Moreover, if we have $V \in \operatorname{Rep}_{\mathrm{st}}^{\nabla} G_{K}$, then the canonical map $K_{\text {can }} \otimes_{K_{\text {can }, 0}} \mathbb{D}_{\mathrm{st}}^{\nabla}(V) \rightarrow \mathbb{D}_{\mathrm{dR}}^{\nabla}(V)$ is an isomorphism of filtered $K_{\text {can }}$-modules. Finally, let $V \in \operatorname{Rep}_{\mathrm{dR}}^{\nabla} G_{K}$. We choose a

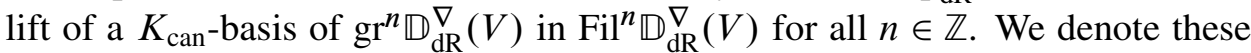
lifts by $\left\{e_{i}\right\}$ and let $n_{i} \in \mathbb{Z}$ such that $e_{i} \in \mathrm{Fil}^{n_{i}} \mathbb{D}_{\mathrm{dR}}^{\nabla}(V) \backslash \mathrm{Fil}^{n_{i}+1} \mathbb{D}_{\mathrm{dR}}^{\nabla}(V)$. Then, $\left\{e_{i}\right\}$

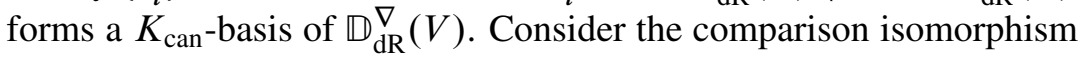

$$
\mathbb{B}_{\mathrm{dR}}^{\nabla} \otimes_{K_{\mathrm{can}}} \mathbb{D}_{\mathrm{dR}}^{\nabla}(V) \rightarrow \mathbb{B}_{\mathrm{dR}}^{\nabla} \otimes_{\mathbb{Q}_{p}} V
$$

which is compatible with the filtrations. By taking Fil ${ }^{n}$ of both sides, we have

$$
\sum_{i} t^{n-n_{i}} \mathbb{B}_{\mathrm{dR}}^{\nabla+} e_{i}=t^{n} \mathbb{B}_{\mathrm{dR}}^{\nabla+} \otimes_{\mathbb{Q}_{p}} V
$$

By taking $\mathrm{gr}^{n}$ of both sides and taking $H^{0}\left(G_{K},-\right)$, we have

$$
K \otimes_{K_{\mathrm{can}}} \mathrm{gr}^{n} \mathbb{D}_{\mathrm{dR}}^{\nabla}(V) \cong \bigoplus_{i: n_{i}=n} K e_{i} \cong\left(\mathbb{C}_{p}(n) \otimes_{\mathbb{Q}_{p}} V\right)^{G_{K}}
$$

by Theorem 1.15. Hence, we have an isomorphism $K \otimes_{K_{\text {can }}} \operatorname{gr} \mathbb{D}_{\mathrm{dR}}^{\nabla}(V) \cong \mathbb{D}_{\mathrm{HT}}^{\nabla}(V)$ of filtered $K$-vector spaces, which implies $V \in \operatorname{Rep}_{\mathrm{HT}}^{\nabla} G_{K}$ by Lemma 1.19. In particular, the multiset of Hodge-Tate weights of $V$ coincides with the multiset consisting of $n \in \mathbb{Z}$ with multiplicity $\operatorname{dim}_{K_{\mathrm{can}}} \mathrm{Fil}^{-n} \mathbb{D}_{\mathrm{dR}}^{\nabla}(V) / \mathrm{Fil}^{-n+1} \mathbb{D}_{\mathrm{dR}}^{\nabla}(V)$.

Proposition 7.3. The functors $\mathbb{D}_{\text {cris }}^{\nabla}$ and $\mathbb{D}_{\mathrm{st}}^{\nabla}$ induce the functors

$$
\mathbb{D}_{\text {cris }}^{\nabla}: \operatorname{Rep}_{\text {cris }}^{\nabla} G_{K} \rightarrow M F_{K_{\text {can }}^{\text {wa }}}^{\text {wa }}(\varphi), \quad \mathbb{D}_{\text {st }}^{\nabla}: \operatorname{Rep}_{\text {st }}^{\nabla} G_{K} \rightarrow M F_{K_{\text {can }}}^{\text {wa }}(\varphi, N) .
$$

Moreover, these functors are fully faithful.

Proof. We first prove the weak admissibilities of the images. As noted in Remark 7.2, if $V$ is horizontal crystalline, then $\mathbb{D}_{\text {cris }}^{\nabla}(V)$ coincides with $\mathbb{D}_{\text {st }}^{\nabla}(V)$ as a filtered $(\varphi, N)$-module relative to $K_{\text {can }}$. Therefore, we may reduce to the case that $V$ is horizontal semistable.

For a filtered $(\varphi, N)$-module $D$ relative to $K_{\text {can }}$, we endow the finite $K_{0}^{\mathrm{pf}}$-vector space $D_{K_{0}^{\mathrm{pf}}}$ with a structure of a filtered $(\varphi, N)$-module relative to $K^{\mathrm{pf}}$ as follows. 
We extend the Frobenius $\varphi$ on $D$ to $D_{K_{0}^{\mathrm{pf}}}$ semilinearly and extend the monodromy operator $N$ on $D$ to $D_{K_{0}^{\mathrm{pf}}}$ linearly. We also define a filtration of $D_{K^{\mathrm{pf}}}$ as Fil ${ }^{\bullet} D_{K^{\mathrm{pf}}}:=$ $K^{\mathrm{pf}} \otimes_{K_{\text {can }}}$ Fil $^{\bullet} D_{K_{\text {can }}}$. Moreover, the scalar extension

$$
K_{0}^{\mathrm{pf}} \otimes_{K_{\text {can }, 0}}(-): M F_{K_{\text {can }}}(\varphi, N) \rightarrow M F_{K^{\mathrm{pf}}}(\varphi, N)
$$

induces a functor. We have only to prove that the following diagram is commutative:

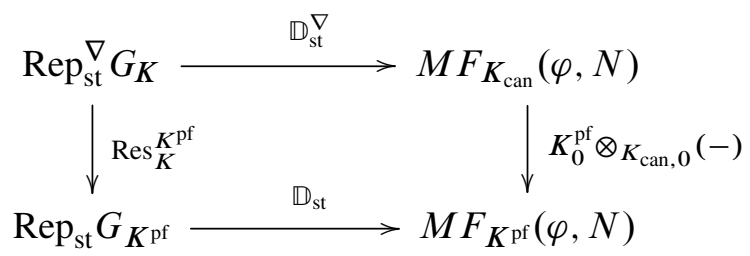

In fact, since $\mathbb{D}_{\mathrm{st}}\left(\left.V\right|_{K^{\mathrm{pf}}}\right)=K_{0}^{\mathrm{pf}} \otimes_{K_{\text {can }, 0}} \mathbb{D}_{\mathrm{st}}^{\nabla}(V)$ is weakly admissible by [Fontaine 1994 b, Proposition 5.4.2(i)], $\mathbb{D}_{\mathrm{st}}^{\nabla}(V)$ is weakly admissible by definition.

By functoriality, the canonical map $i: K_{0}^{\mathrm{pf}} \otimes_{K_{\text {can }, 0}} \mathbb{D}_{\mathrm{st}}^{\nabla}(V) \rightarrow \mathbb{D}_{\mathrm{st}}\left(\left.V\right|_{K^{\mathrm{pf}}}\right)$ is a morphism of filtered $(\varphi, N)$-modules relative to $K_{0}^{\mathrm{pf}}$. Consider the associated graded homomorphism after applying $K^{\mathrm{pf}} \otimes K_{0}^{\mathrm{pf}}$. The resulting homomorphism coincides with the canonical map $K^{\mathrm{pf}} \otimes_{K} \mathbb{D}_{\mathrm{HT}}^{\nabla}(V) \rightarrow \mathbb{D}_{\mathrm{HT}}\left(\left.V\right|_{K \text { pf }}\right)$. Since $V \in \operatorname{Rep}_{\mathrm{HT}}^{\nabla} G_{K}$ by Remark 7.2, a Hodge-Tate decomposition $\mathbb{C}_{p} \otimes_{\mathbb{Q}_{p}} V \cong \bigoplus_{n \in \mathbb{N}} \mathbb{C}_{p}(n)^{m_{n}}$ of $V$ induces a Hodge-Tate decomposition of $\left.V\right|_{K^{\text {pf }}}$. In particular, $\left.V\right|_{K^{\text {pf }}}$ is also HodgeTate and the above canonical map is an isomorphism. Since the filtrations of $\mathbb{D}_{\mathrm{st}}^{\nabla}(V)$ and $\mathbb{D}_{\mathrm{st}}\left(\left.V\right|_{K^{\mathrm{pf}}}\right)$ are separated and exhaustive, $i$ is an isomorphism as filtered $(\varphi, N)$ modules relative to $K_{0}^{\mathrm{pf}}$.

We prove the full faithfulness. We have the fundamental exact sequence

$$
0 \longrightarrow \mathbb{Q}_{p} \stackrel{\text { inc. }}{\longrightarrow}\left(\mathbb{B}_{\text {cris }}^{\nabla}\right)^{\varphi=1} \stackrel{\text { can. }}{\longrightarrow} \mathbb{B}_{\mathrm{dR}}^{\nabla} / \mathbb{B}_{\mathrm{dR}}^{\nabla+} \longrightarrow 0 .
$$

Indeed, the exactness is reduced to [Colmez and Fontaine 2000, Proposition 9.25] by

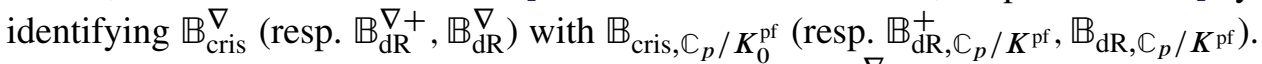
By the fundamental exact sequence, we have $\mathbb{V}_{\text {st }} \circ \mathbb{D}_{\text {st }}^{\nabla}(V)=V$ (resp. $\mathbb{V}_{\text {cris }} \circ$ $\mathbb{D}_{\text {cris }}^{\nabla}(V)=V$ ) for $V \in \operatorname{Rep}_{\text {st }}^{\nabla} G_{K}$ (resp. $V \in \operatorname{Rep}_{\text {cris }}^{\nabla} G_{K}$ ). This implies the full faithfulness.

In Proposition 7.5(ii), we will prove that the above functors induce equivalences of categories, that is, are essentially surjective.

7B. A horizontal analogue of the p-adic monodromy theorem. The following is a horizontal analogue of the $p$-adic monodromy theorem. Note that the converse is true by Hilbert 90 and Corollary 4.3.

Theorem 7.4. Let $V \in \operatorname{Rep}_{\mathrm{dR}}^{\nabla} G_{K}$. Then, there exists a finite extension $K^{\prime} / K_{\mathrm{can}}$ such that $\left.V\right|_{K K^{\prime}}$ is horizontal semistable. 
Proof. First, the comparison isomorphism $\alpha_{\mathrm{dR}, \mathbb{C}_{p} / K}^{\nabla}$ induces an isomorphism of $\mathbb{B}_{\mathrm{dR}, \mathbb{C}_{p} / K}\left[G_{K}\right]$-modules

$$
\mathbb{B}_{\mathrm{dR}, \mathbb{C}_{p} / K} \otimes_{K_{\mathrm{can}}} \mathbb{D}_{\mathrm{dR}}^{\nabla}(V) \rightarrow \mathbb{B}_{\mathrm{dR}, \mathbb{C}_{p} / K} \otimes_{\mathbb{Q}_{p}} V
$$

By taking $H^{0}\left(G_{K},-\right)$, we have $\operatorname{dim}_{K} \mathbb{D}_{\mathrm{dR}}(V)=\operatorname{dim}_{\mathbb{Q}_{p}} V$ by Corollary 4.3 , which implies $V \in \operatorname{Rep}_{\mathrm{dR}} G_{K}$ by Lemma 1.19 . Hence, there exists a finite extension $L / K$ such that $\left.V\right|_{L}$ is semistable by the Main Theorem. We may assume that $L / K$ is a finite Galois extension satisfying condition $(\mathrm{H})$ by the proof of the Main Theorem (Step 1) and Epp's Theorem 1.6. The extension $L_{\text {can }} / K_{\text {can }}$ is finite Galois by Lemma 1.5(ii). We will prove the assertion for $K^{\prime}=L_{\text {can }}$.

We have canonical isomorphisms

$$
L_{\text {can }} \otimes_{L_{\text {can }, 0}} \mathbb{D}_{\mathrm{st}}\left(\left.V\right|_{L}\right) \cong L \otimes_{L_{0}} \mathbb{D}_{\mathrm{st}}\left(\left.V\right|_{L}\right) \cong \mathbb{D}_{\mathrm{dR}}\left(\left.V\right|_{L}\right),
$$

where the first one is induced by a canonical isomorphism $L_{\text {can }} \otimes_{L_{\text {can, } 0}} L_{0} \rightarrow L$ (Remark 1.4(ii)), the second one follows by using Lemma 1.20 and Proposition 3.16. Moreover, these maps are compatible with the residual $G_{L / K}$-actions and the $\nabla$ actions. By taking the horizontal sections, we have

$$
\begin{aligned}
\mathbb{D}_{\mathrm{dR}}^{\nabla}\left(\left.V\right|_{L}\right) & \cong \mathbb{D}_{\mathrm{dR}}\left(\left.V\right|_{L}\right)^{\nabla=0} \cong\left(L_{\mathrm{can}} \otimes_{L_{\mathrm{can}, 0}} \mathbb{D}_{\mathrm{st}}\left(\left.V\right|_{L}\right)\right)^{\nabla=0} \\
& \cong L_{\text {can }} \otimes_{L_{\mathrm{can}, 0}} \mathbb{D}_{\mathrm{st}}\left(\left.V\right|_{L}\right)^{\nabla=0} \cong L_{\text {can }} \otimes_{L_{\text {can }, 0}} \mathbb{D}_{\mathrm{st}}^{\nabla}\left(\left.V\right|_{L}\right),
\end{aligned}
$$

where the third equality follows from the fact $\left.\nabla\right|_{L_{\text {can }}}=0$. By taking $G_{L / K} \cdot L_{\text {can }}$ invariants, we have $\mathbb{D}_{\mathrm{dR}}^{\nabla}\left(\left.V\right|_{K \cdot L_{\text {can }}}\right)=L_{\text {can }} \otimes_{L_{\text {can }, 0}} \mathbb{D}_{\mathrm{st}}^{\nabla}\left(\left.V\right|_{K \cdot L_{\text {can }}}\right)$. Since $\left.V\right|_{K \cdot L_{\text {can }}}$ is horizontal de Rham by Remark 1.22 and since $\left(K \cdot L_{\text {can }}\right)_{\text {can }}=L_{\text {can }}$ by Lemma 1.5(iv), we have

$$
\operatorname{dim}_{L_{\text {can }}} \mathbb{D}_{\mathrm{dR}}^{\nabla}\left(\left.V\right|_{K \cdot L_{\text {can }}}\right)=\operatorname{dim}_{\mathbb{Q}_{p}} V=\operatorname{dim}_{L_{\text {can }, 0}} \mathbb{D}_{\mathrm{st}}^{\nabla}\left(\left.V\right|_{K \cdot L_{\text {can }}}\right),
$$

which implies that $\left.V\right|_{K \cdot L_{\text {can }}}$ is horizontal semistable.

7C. Equivalences of categories. The surjection of profinite groups $\imath^{*}: G_{K} \rightarrow$ $G_{K_{\text {can }}}$ induces a $\otimes$-functor of Tannakian categories

$$
l^{*}: \operatorname{Rep}_{\mathbb{Q}_{p}} G_{K_{\text {can }}} \rightarrow \operatorname{Rep}_{\mathbb{Q}_{p}} G_{K} .
$$

Obviously, the functor $\iota^{*}$ is fully faithful. Denote by $\boldsymbol{C}_{p}$ the $p$-adic completion of the algebraic closure of $K_{\text {can }}$ in $\bar{K}$. For $\bullet \in\{$ cris, st, dR $\}$, we have a Galois equivariant canonical injection $\mathbb{B}_{\bullet}, C_{p} / K_{\text {can }} \rightarrow \mathbb{B}_{\bullet}^{\nabla}, \mathbb{C}_{p} / K$ by functoriality and we have $\left.\left(\mathbb{B}_{\bullet}, C_{p} / K_{\text {can }}\right)^{G_{K_{\text {can }}} \cong\left(\mathbb{B}_{\bullet}^{\nabla} \mathbb{C}_{p} / K\right.}\right)^{G_{K}}\left(=K_{\text {can }}\right.$ if $\bullet=\mathrm{dR}, K_{\text {can }, 0}$ otherwise $)$ by Proposition 3.16. Hence, if we have $V \in \operatorname{Rep} . G_{K_{\text {can }}}$, then we have $\imath^{*} V \in \operatorname{Rep}^{\nabla} G_{K}$. In fact, we have a canonical injection $\mathbb{D}_{\bullet}(V) \subset \mathbb{D}_{\bullet}^{\nabla}\left(\imath^{*} V\right)$ of $\left(\mathbb{B}_{\bullet}, C_{p} / K_{\text {can }}\right)^{G_{K_{\text {can }}}}$

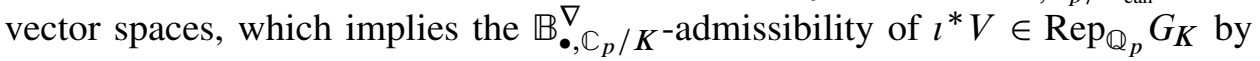


Lemma 1.19. Hence, $\imath^{*}$ induces a fully faithful $\otimes$-functor

$$
\imath_{\bullet}^{*}: \operatorname{Rep}_{\bullet} G_{K_{\text {can }}} \rightarrow \operatorname{Rep}_{\bullet}^{\nabla} G_{K} .
$$

The following proposition is a direct consequence of théorème 4.3 in [Colmez and Fontaine 2000].

Proposition 7.5 (horizontal analogue of Colmez-Fontaine). (i) The functors $l_{\text {cris }}^{*}$ and $l_{\mathrm{st}}^{*}$ are essentially surjective. In particular, $l_{\mathrm{cris}}^{*}$ and $l_{\mathrm{st}}^{*}$ induce equivalences of Tannakian categories.

(ii) The functors

$$
\mathbb{D}_{\text {cris }}^{\nabla}: \operatorname{Rep}_{\text {cris }}^{\nabla} G_{K} \rightarrow M F_{K_{\text {can }}}^{\mathrm{wa}}(\varphi), \quad \mathbb{D}_{\text {st }}^{\nabla}: \operatorname{Rep}_{\mathrm{st}}^{\nabla} G_{K} \rightarrow M F_{K_{\text {can }}}^{\mathrm{wa}}(\varphi, N)
$$

induce equivalences of categories with quasi-inverses $\mathbb{V}_{\text {cris }}, \mathbb{V}_{\text {st }}$.

Proof. We first prove the assertion in the semistable case. Together with the full faithfulness of $\mathbb{D}_{\text {st }}^{\nabla}$, we have only to prove the commutativity of the diagram

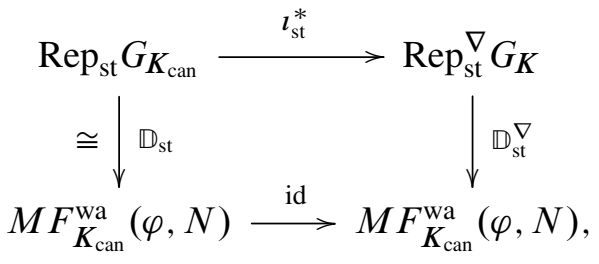

where $\mathbb{D}_{\mathrm{st}}$ is an equivalence of categories by Colmez-Fontaine theorem [2000, Théorème 4.3]. As we mentioned above, the canonical map $\mathbb{D}_{\mathrm{st}}(V) \rightarrow \mathbb{D}_{\mathrm{st}}^{\nabla}\left(l^{*} V\right)$,

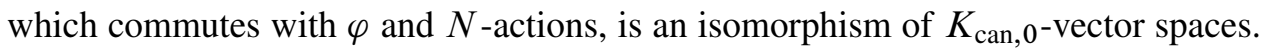
We have only to prove that the map also preserves the filtrations. Obviously, we have Fil ${ }^{\bullet} \mathbb{D}_{\mathrm{st}}(V) \subset \mathrm{Fil}^{\bullet} \mathbb{D}_{\mathrm{st}}^{\nabla}\left(l^{*} V\right)$. To prove the converse, it suffices to prove that the associated graded modules of both sides have the same dimension since the filtrations are exhaustive and separated. Let $C_{p} \otimes_{\mathbb{Q}_{p}} V \cong \bigoplus_{n \in \mathbb{Z}} C_{p}(n)^{m_{n}}$ be the Hodge-Tate decomposition of $V$. Then, it induces the Hodge-Tate decomposition of $l^{*} V$, that is, $\mathbb{C}_{p} \otimes_{\mathbb{Q}_{p}} l^{*} V \cong \bigoplus_{n \in \mathbb{Z}} \mathbb{C}_{p}(n)^{m_{n}}$, which implies the assertion.

In the horizontal crystalline case, a similar proof works by replacing $*_{\mathrm{st}}$ and $M F_{K_{\text {can }}}^{\mathrm{wa}}(\varphi, N)$ by $*_{\text {cris }}$ and $M F_{K_{\text {can }}}^{\mathrm{wa}}(\varphi)$.

Theorem 7.6. The functor $l_{\mathrm{dR}}^{*}$ is essentially surjective. In particular, $l_{\mathrm{dR}}^{*}$ induces an equivalence of Tannakian categories.

Proof. For a finite Galois extension $L / K$ such that $K \cdot L_{\text {can }}=L$, let $\mathscr{C}_{L / K}$ be the full subcategory of $\operatorname{Rep}_{\mathrm{dR}}^{\nabla} G_{K}$ whose objects consist of $V \in \operatorname{Rep}_{\mathrm{dR}}^{\nabla} G_{K}$ such that $\left.V\right|_{L}$ is horizontal semistable. Recall the notation in Defintion 7.1. Then, we have an equivalence of categories

$$
\mathbb{D}_{\mathrm{st}, L}^{\nabla}: \mathscr{C}_{L / K} \rightarrow M F^{\mathrm{wa}}\left(\varphi, N, G_{L / K}\right) ; \quad V \mapsto \mathbb{D}_{\mathrm{st}}^{\nabla}\left(\left.V\right|_{L}\right)
$$


In fact, we have the following quasi-inverse $\mathbb{V}_{\mathrm{st}, L}$ : For $D \in M F^{\mathrm{wa}}\left(\varphi, N, G_{L / K}\right)$, we regard $D$ as an object of $M F_{L_{\text {can }}}^{\text {wa }}(\varphi, N)$ and let $\mathbb{V}_{\mathrm{st}, L}(D):=\mathbb{V}_{\mathrm{st}}(D)$. We have $\mathbb{V}_{\text {st }, L}(D) \in \operatorname{Rep}_{\mathrm{st}}^{\nabla} G_{L}$ by Proposition 7.5(ii) and $\mathbb{V}_{\mathrm{st}, L}(D)$ has a canonical $G_{K}$-action, which is an extension of the action of $G_{L}$, induced by the $G_{L / K}$-action on $D$. We have $D \in \mathscr{C}_{L / K}$ by Remark 4.8 and Remark 7.2. We have $\mathbb{V}_{\mathrm{st}, L} \circ \mathbb{D}_{\mathrm{st}, L}^{\nabla} \cong \mathrm{id}_{\mathscr{C}_{L / K}}$ and $\mathbb{D}_{\mathrm{st}, L}^{\nabla} \circ \mathbb{V}_{\mathrm{st}, L} \cong \mathrm{id}_{M F^{\mathrm{wa}}\left(\varphi, N, G_{L / K}\right)}$ by Proposition 7.5(ii).

The restriction map $\operatorname{Res}_{L_{\text {can }}}^{L}: G_{L / K} \stackrel{\cong}{\rightarrow} G_{L_{\text {can }} / K_{\text {can }}}$ induces the equivalence of categories

$$
\left(\operatorname{Res}_{L_{\text {can }}}^{L}\right)^{*}: M F^{\mathrm{wa}}\left(\varphi, N, G_{L_{\text {can }} / K_{\text {can }}}\right) \stackrel{\cong}{\longrightarrow} M F^{\mathrm{wa}}\left(\varphi, N, G_{L / K}\right)
$$

We will prove that the diagram

$$
\begin{aligned}
& M F^{\mathrm{wa}}\left(\varphi, N, G_{L_{\mathrm{can}} / K_{\mathrm{can}}}\right) \stackrel{\left(\operatorname{Res}_{L_{\mathrm{can}}}^{L}\right)^{*}}{\cong} M F^{\mathrm{wa}}\left(\varphi, N, G_{L / K}\right) \\
& \begin{array}{ccc}
\cong \downarrow \nabla_{\mathrm{st}, L \text { can }} & & \cong \downarrow \nabla_{\mathrm{st}, L}^{\nabla} \\
\mathscr{C}_{L_{\text {can }} / K_{\text {can }}} \longrightarrow \mathscr{C}_{L / K}^{*}
\end{array}
\end{aligned}
$$

is commutative, where the bottom horizontal arrow is induced by $\imath_{\mathrm{dR}}^{*}: \operatorname{Rep}_{\mathrm{dR}} G_{K_{\text {can }}} \rightarrow$ $\operatorname{Rep}_{\mathrm{dR}}^{\nabla} G_{K}$. Indeed, we have the $G_{K}$-equivariant inclusion

$$
l_{\mathrm{dR}}^{*} \circ \mathbb{V}_{\mathrm{st}, L_{\mathrm{can}}}(D) \subset \mathbb{V}_{\mathrm{st}, L}^{\nabla} \circ\left(\operatorname{Res}_{L_{\mathrm{can}}}^{L}\right)^{*}(D)
$$

for $D \in M F^{\text {wa }}\left(\varphi, N, G_{L_{\text {can }} / K_{\text {can }}}\right)$ by construction. Since both sides have the same dimension over $\mathbb{Q}_{p}$, this inclusion is an equality. By the commutative diagram, the

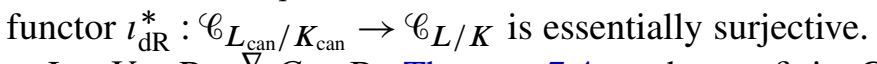

Let $V \in \operatorname{Rep}_{\mathrm{dR}}^{\nabla} G_{K}$. By Theorem 7.4, we have a finite Galois extension $K^{\prime} / K_{\text {can }}$ such that $\left.V\right|_{G_{K K^{\prime}}}$ is horizontal semistable. Let $L:=K K^{\prime}$. By Lemma 1.5(iv), we have $L_{\text {can }}=K^{\prime}$, that is, $L / K$ satisfies the above assumption. Since we have $V \in$ $\mathscr{C}_{L / K}$, the assertion follows from the essential surjectivity of

$$
l_{\mathrm{dR}}^{*}: \mathscr{C}_{L_{\mathrm{can}} / K_{\mathrm{can}}} \rightarrow \mathscr{C}_{L / K} .
$$

The above equivalence induces a $\mathbb{Q}_{p}$-linear isomorphism of $\operatorname{Ext}^{1}$ on $\operatorname{Rep}_{\mathrm{dR}} G_{K_{\text {can }}}$

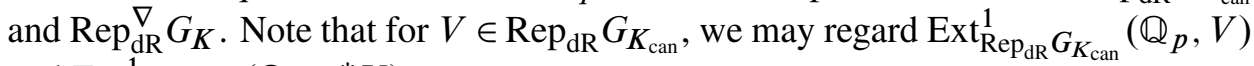
and $\operatorname{Ext}_{\mathrm{Rep}_{\mathrm{dR}}^{1} \nabla G_{K}}^{\nabla}\left(\mathbb{Q}_{p}, l^{*} V\right)$ as

$$
\begin{aligned}
H_{g}^{1}\left(G_{K_{\mathrm{can}}}, V\right) & :=\operatorname{ker}\left(H^{1}\left(G_{K}, V\right) \stackrel{(1 \otimes \mathrm{id})_{*}}{\longrightarrow} H^{1}\left(G_{K}, \mathbb{B}_{\mathrm{dR}, \mathbb{C}_{p} / K_{\mathrm{can}}} \otimes_{\mathbb{Q}_{p}} V\right)\right), \\
H_{g}^{1, \nabla}\left(G_{K}, l^{*} V\right) & :=\operatorname{ker}\left(H^{1}\left(G_{K}, l^{*} V\right) \stackrel{(1 \otimes \mathrm{id})_{*}}{\longrightarrow} H^{1}\left(G_{K}, \mathbb{B}_{\mathrm{dR}, \mathbb{C}_{p} / K}^{\nabla} \otimes_{\mathbb{Q}_{p}} l^{*} V\right)\right)
\end{aligned}
$$

respectively. In particular: 
Corollary 7.7. For $V \in \operatorname{Rep}_{\mathrm{dR}} G_{K_{\mathrm{can}}}$, the inflation map

$$
\text { Inf : } H^{1}\left(G_{K_{\text {can }}}, V\right) \rightarrow H^{1}\left(G_{K}, \imath^{*} V\right)
$$

induces the isomorphism

$$
\text { Inf : } H_{g}^{1}\left(G_{K_{\text {can }}}, V\right) \cong H_{g}^{1, \nabla}\left(G_{K}, l^{*} V\right) .
$$

7D. A comparison theorem on $\boldsymbol{H}^{\mathbf{1}}$. Notation is as in the previous subsection.

Theorem 7.8 (a generalization of Theorem 1.16). Let $V \in \operatorname{Rep}_{\mathbb{Q}_{p}} G_{K_{\text {can }}}$ be $a$ de Rham representation whose Hodge-Tate weights are greater than or equal to 1 . Then, we have the exact sequence

$$
0 \rightarrow H^{1}\left(G_{K_{\text {can }}}, V\right) \stackrel{\operatorname{Inf}}{\longrightarrow} H^{1}\left(G_{K}, l^{*} V\right) \stackrel{(1 \otimes \mathrm{id})_{*}}{\longrightarrow} H^{1}\left(G_{K}, \mathbb{C}_{p} \otimes_{\mathbb{Q}_{p}} \iota^{*} V\right)
$$

and a canonical isomorphism

$$
\left(C_{p} \otimes_{\mathbb{Q}_{p}} V(-1)\right)^{G_{K_{\text {can }}}} \otimes_{K_{\text {can }}} \hat{\Omega}_{K}^{1} \cong H^{1}\left(G_{K}, \mathbb{C}_{p} \otimes_{\mathbb{Q}_{p}} l^{*} V\right) .
$$

Moreover, if the Hodge-Tate weights of $V$ are greater than or equal to 2, then $H^{1}\left(G_{K}, \mathbb{C}_{p} \otimes_{\mathbb{Q}_{p}} l^{*} V\right)$ vanishes, in particular, the inflation map

$$
\text { Inf : } H^{1}\left(G_{K_{\text {can }}}, V\right) \rightarrow H^{1}\left(G_{K}, \imath^{*} V\right)
$$

is an isomorphism.

Proof. We first prove the exactness of (7). Note that the injectivity of the inflation map follows by definition. We have the commutative diagram

$$
\begin{gathered}
H^{1}\left(G_{K_{\text {can }}}, V\right) \\
\downarrow^{1}(1 \otimes \mathrm{id})_{*} \\
H^{1}\left(G_{K_{\text {can }}}, C_{p} \otimes_{\mathbb{Q}_{p}} V\right) \stackrel{\text { Inf }}{\longrightarrow} H^{1}\left(G_{K}, \mathbb{C}_{p} \otimes_{\mathbb{Q}_{p}} l^{*} V\right) .
\end{gathered}
$$

Since we have a Hodge-Tate decomposition $C_{p} \otimes_{\mathbb{Q}_{p}} V \cong \bigoplus_{n \in \mathbb{N}_{\geq 1}} C_{p}(n)^{m_{n}}$, we have $H^{1}\left(G_{K_{\text {can }}}, C_{p} \otimes_{\mathbb{Q}_{p}} V\right)=0$ by Theorem 1.15 , which implies $(1 \otimes \mathrm{id})_{*} \circ \mathrm{Inf}=0$.

Let $\mathscr{H}:=\operatorname{ker}\left\{(1 \otimes \mathrm{id})_{*}: H^{1}\left(G_{K}, l^{*} V\right) \rightarrow H^{1}\left(G_{K}, \mathbb{C}_{p} \otimes_{\mathbb{Q}_{p}} l^{*} V\right)\right\}$. We have only to prove $\mathscr{H}$ is contained in the image of Inf: $H^{1}\left(G_{K_{\text {can }}}, V\right) \rightarrow H^{1}\left(G_{K}, l^{*} V\right)$. Consider the exact sequence

$$
0 \longrightarrow t \mathbb{B}_{\mathrm{dR}, \mathbb{C}_{p} / K}^{\nabla+} \stackrel{\text { inc. }}{\longrightarrow} \mathbb{B}_{\mathrm{dR}, \mathbb{C}_{p} / K}^{\nabla+} \stackrel{\theta}{\longrightarrow} \mathbb{C}_{p} \longrightarrow 0
$$

with $\theta:=\theta_{\mathbb{C}_{p} / K}$. By applying $\otimes_{\mathbb{Q}_{p}} l^{*} V$ and taking $H^{\bullet}\left(G_{K},-\right)$, we have the 
commutative diagram with exact row, where $S$ stands for $\mathbb{B}_{\mathrm{dR}, \mathbb{C}_{p} / K}^{\nabla+}$ :

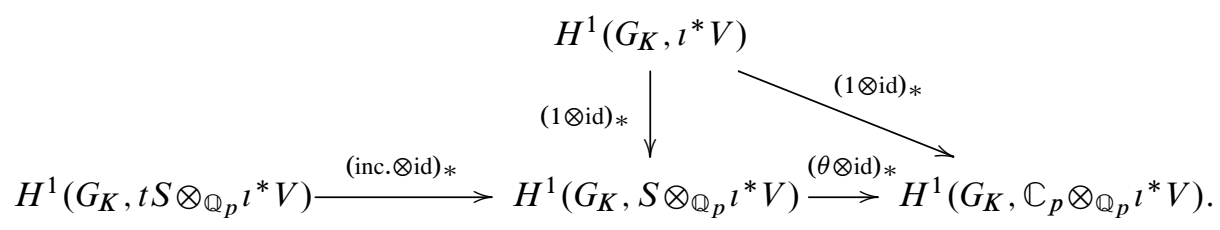

Since $V(1)$ is de Rham with Hodge-Tate weights $\geq 2$, we have

$$
H^{1}\left(G_{K}, t \mathbb{B}_{\mathrm{dR}, \mathbb{C}_{p} / K}^{\nabla+} \otimes_{\mathbb{Q}_{p}} l^{*} V\right)=0
$$

by Theorem 1.15, Lemma 1.14 and dévissage. Hence, the canonical map

$$
(1 \otimes \mathrm{id})_{*}: \mathscr{H} \rightarrow H^{1}\left(G_{K}, \mathbb{B}_{\mathrm{dR}, \mathbb{C}_{p} / K}^{\nabla+} \otimes_{\mathbb{Q}_{p}} l^{*} V\right)
$$

vanishes by the above exact sequence. In particular, we have $\mathscr{H} \subset H_{g}^{1, \nabla}\left(G_{K}, l^{*} V\right)$. By Corollary 7.7, we have Inf : $H_{g}^{1}\left(G_{K_{\text {can }}}, V\right) \cong H_{g}^{1, \nabla}\left(G_{K}, l^{*} V\right)$, which implies (7).

Then, we will prove the existence of the canonical isomorphism (8). By the inclusion $\left(\boldsymbol{C}_{p} \otimes_{\mathbb{Q}_{p}} V(-1)\right)^{G_{K_{\text {can }}}} \subset\left(\mathbb{C}_{p} \otimes_{\mathbb{Q}_{p}} l^{*} V(-1)\right)^{G_{K}}$ and the canonical isomorphism $\hat{\Omega}_{K}^{1} \rightarrow H^{1}\left(G_{K}, \mathbb{C}_{p}(1)\right)$ in Theorem 1.15 , we can define a canonical map $f$ as the composite

$$
\begin{aligned}
& \left(C_{p} \otimes_{\mathbb{Q}_{p}} V(-1)\right)^{G_{K_{\text {can }}}} \otimes_{K_{\text {can }}} \hat{\Omega}_{K}^{1} \stackrel{\text { inc. } \otimes \text { can. }}{\longrightarrow} \\
& \quad\left(\mathbb{C}_{p} \otimes_{\mathbb{Q}_{p}} l^{*} V(-1)\right)^{G_{K}} \otimes_{K} H^{1}\left(G_{K}, \mathbb{C}_{p}(1)\right) \stackrel{\text { cup. }}{\longrightarrow} H^{1}\left(G_{K}, \mathbb{C}_{p} \otimes_{\mathbb{Q}_{p}} l^{*} V\right) .
\end{aligned}
$$

We will prove that $f$ is an isomorphism. A Hodge-Tate decomposition of $V$ induces a Hodge-Tate decomposition $\mathbb{C}_{p} \otimes_{\mathbb{Q}_{p}} l^{*} V \cong \bigoplus_{n \in \mathbb{N}_{\geq 1}} \mathbb{C}_{p}(n)^{m_{n}}$ of $l^{*} V$. By replacing $C_{p} \otimes_{\mathbb{Q}_{p}} V$ and $\mathbb{C}_{p} \otimes_{\mathbb{Q}_{p}} l^{*} V$ by their Hodge-Tate decompositions, we may reduce to the case $V=\mathbb{Q}_{p}(n)$ with $n \in \mathbb{N}_{\geq 1}$ since the cup product commutes with direct sums. Then, the assertion follows from Theorem 1.15.

We will prove the last assertion. The assumption implies that we have $m_{1}=0$ in the above notation, hence, we have $H^{1}\left(G_{K}, \mathbb{C}_{p} \otimes_{\mathbb{Q}_{p}} \imath^{*} V\right)=0$ by the Hodge-Tate decomposition of $\imath^{*} V$ and Theorem 1.15.

Remark 7.9. (i) Originally, Theorem 1.16(i) and (ii) are proved separately by using ramification theory in some sense.

(ii) (Finiteness) Suppose that we have $\left[K_{\text {can }}: \mathbb{Q}_{p}\right]<\infty$. For example, consider the case that $K$ has a structure of a higher-dimensional local field (Example 1.7). Let $V \in \operatorname{Rep}_{\mathbb{Q}_{p}} G_{K}$ be horizontal de Rham of Hodge-Tate weights greater than or equal to 2 . Then we have

$$
\operatorname{dim}_{\mathbb{Q}_{p}} H^{1}\left(G_{K}, V\right)=\left[K_{\text {can }}: \mathbb{Q}_{p}\right] \operatorname{dim}_{\mathbb{Q}_{p}} V<\infty .
$$


Indeed, by Theorem 7.6 and 7.8, we may reduce to the case $K=K_{\text {can }}$. By a Hodge-Tate decomposition $\mathbb{C}_{p} \otimes_{\mathbb{Q}_{p}} V \cong \bigoplus_{n \in \mathbb{N}_{>2}} \mathbb{C}_{p}(n)^{m_{n}}$ with $m_{n} \in \mathbb{N}$, we have $H^{0}\left(G_{K}, V\right) \subset H^{0}\left(G_{K}, \mathbb{C}_{p} \otimes_{\mathbb{Q}_{p}} V\right)=0$ and $H^{2}\left(G_{K}, V\right) \cong H^{0}\left(G_{K}, V^{\vee}(1)\right) \subset$ $H^{0}\left(G_{K}, \mathbb{C}_{p} \otimes_{\mathbb{Q}_{p}} V^{\vee}(1)\right)=0$ by the local Tate duality [Herr 1998, Théorème in Introduction], where ${ }^{\vee}$ denotes the dual. Then, the assertion follows from the Euler-Poincaré characteristic formula (loc. cit).

Note that $H^{1}\left(G_{K}, V\right)$ is not finite over $\mathbb{Q}_{p}$ without the condition on HodgeTate weights: For example, $H^{1}\left(G_{K}, \mathbb{Q}_{p}(1)\right) \cong \mathbb{Q}_{p} \otimes_{\mathbb{Z}_{p}} \lim _{n} K^{\times} /\left(K^{\times}\right)^{p^{n}}$ contains $\mathbb{Q}_{p} \otimes_{\mathbb{Z}_{p}} \mathbb{O}_{K}$, which is infinite-dimensional over $\mathbb{Q}_{p}$ if $k_{K}^{n}$ is imperfect, via the map $\mathrm{O}_{K} \hookrightarrow U_{K}^{(1)}$ that takes $x$ to $\exp (2 p x)$.

\section{Acknowledgment}

The author thanks his advisor Atsushi Shiho for reading earlier manuscripts carefully. The author also thanks Professor Takeshi Tsuji for pointing out errors in an earlier manuscript and thanks Professor Olivier Brinon and Kazuma Morita for helpful discussions. The author thanks the referee for detailed comments.

\section{References}

[Andreatta and Brinon 2010] F. Andreatta and O. Brinon, " $B_{d R}-$ représentations dans le cas relatif", Ann. Sci. Éc. Norm. Supér. (4) 43:2 (2010), 279-339. MR 2011e:11097 Zbl 1195.11074

[Berger 2002] L. Berger, "Représentations $p$-adiques et équations différentielles", Invent. Math. 148:2 (2002), 219-284. MR 2004a:14022 Zbl 1113.14016

[Berthelot and Ogus 1978] P. Berthelot and A. Ogus, Notes on crystalline cohomology, Princeton University Press, 1978. MR 58 \#10908 Zbl 0383.14010

[Brinon 2006] O. Brinon, "Représentations cristallines dans le cas d'un corps résiduel imparfait", Ann. Inst. Fourier (Grenoble) 56:4 (2006), 919-999. MR 2007h:11131 Zbl 1168.11051

[Colmez 2002] P. Colmez, "Espaces de Banach de dimension finie", J. Inst. Math. Jussieu 1:3 (2002), 331-439. MR 2004b:11160 Zbl 1044.11102

[Colmez 2008] P. Colmez, "Espaces vectoriels de dimension finie et représentations de de Rham", pp. 117-186 in Représentations p-adiques de groupes p-adiques, I: Représentations galoisiennes et $(\phi, \Gamma)-$ modules, edited by L. Berger et al., Astérisque 319, Société Mathématique de France, Paris, 2008. MR 2010d:11137 Zbl 1168.11021

[Colmez and Fontaine 2000] P. Colmez and J.-M. Fontaine, "Construction des représentations padiques semi-stables”, Invent. Math. 140:1 (2000), 1-43. MR 2001g:11184 Zbl 1010.14004

[Epp 1973] H. P. Epp, “Eliminating wild ramification”, Invent. Math. 19 (1973), 235-249. MR 48 \#294 Zbl 0254.13008

[Fesenko and Vostokov 2002] I. B. Fesenko and S. V. Vostokov, Local fields and their extensions, 2nd ed., Translations of Mathematical Monographs 121, Amer. Math. Soc., Providence, RI, 2002. MR 2003c:11150 Zbl 1156.11046

[Fontaine 1994a] J.-M. Fontaine, "Le corps des périodes p-adiques", pp. 59-111 in Périodes padiques (Bures-sur-Yvette, 1988), Astérisque 223, Société Mathématique de France, Paris, 1994. MR 95k:11086 Zbl 0940.14012 
[Fontaine 1994b] J.-M. Fontaine, "Représentations p-adiques semi-stables", pp. 113-184 in Périodes p-adiques (Bures-sur-Yvette, 1988), Astérisque 223, Société Mathématique de France, Paris, 1994. MR 95g:14024 Zbl 0865.14009

[Grothendieck 1964] A. Grothendieck, "Éléments de géométrie algébrique, IV: Étude locale des schémas et des morphismes de schémas, I", Inst. Hautes Études Sci. Publ. Math. 20 (1964), 1-259. MR 30 \#3885 Zbl 0136.15901

[Herr 1998] L. Herr, "Sur la cohomologie galoisienne des corps p-adiques", Bull. Soc. Math. France 126:4 (1998), 563-600. MR 2000m:11118 Zbl 0967.11050

[Hyodo 1986] O. Hyodo, "On the Hodge-Tate decomposition in the imperfect residue field case", $J$. Reine Angew. Math. 365 (1986), 97-113. MR 87m:14052 Zbl 0571.14004

[Hyodo 1987] O. Hyodo, "Wild ramification in the imperfect residue field case", pp. 287-314 in Galois representations and arithmetic algebraic geometry (Kyoto, 1985/Tokyo, 1986), edited by Y. Ihara, Adv. Stud. Pure Math. 12, North-Holland, Amsterdam, 1987. MR 89j:11116 Zbl 0649.12011

[Morita 2011] K. Morita, "Crystalline and semi-stable representations in the imperfect residue field case”, preprint, 2011. arXiv 1105.0846v2

[Neukirch et al. 2008] J. Neukirch, A. Schmidt, and K. Wingberg, Cohomology of number fields, 2nd ed., Grundlehren der Mathematischen Wissenschaften 323, Springer, Berlin, 2008. MR 2008m:11223 Zbl 1136.11001

[Schneider 2002] P. Schneider, Nonarchimedean functional analysis, Springer, Berlin, 2002. MR 2003a:46106 Zbl 0998.46044

[Scholl 1998] A. J. Scholl, “An introduction to Kato's Euler systems”, pp. 379-460 in Galois representations in arithmetic algebraic geometry (Durham, 1996), edited by A. J. Scholl and R. L. Taylor, London Math. Soc. Lecture Note Ser. 254, Cambridge Univ. Press, 1998. MR 2000g:11057 Zbl 0952.11015

[Sen 1980] S. Sen, "Continuous cohomology and p-adic Galois representations", Invent. Math. 62:1 (1980), 89-116. MR 82e:12018 Zbl 0463.12005

[Weibel 1994] C. A. Weibel, An introduction to homological algebra, Cambridge Studies in Advanced Mathematics 38, Cambridge University Press, 1994. MR 95f:18001 Zbl 0797.18001

[Zhukov 2000] I. Zhukov, "Higher dimensional local fields", pp. 5-18 in Invitation to higher local fields (Münster, 1999), edited by I. Fesenko and M. Kurihara, Geom. Topol. Monogr. 3, Geom. Topol. Publ., Coventry, 2000. MR 2001k:11245 Zbl 1008.11057

Communicated by Brian Conrad

Received 2012-07-01 Revised 2013-04-02 Accepted 2013-05-02

shuno@ms.u-tokyo.ac.jp Department of Mathematical Sciences, University of Tokyo, Tokyo 153-8914, Japan 


\section{Algebra \& Number Theory}

msp.org/ant

\section{EDITORS}

MANAGING EDITOR

Bjorn Poonen

Massachusetts Institute of Technology

Cambridge, USA

\author{
EDITORIAL BOARD CHAIR \\ David Eisenbud \\ University of California \\ Berkeley, USA
}

\section{BOARD OF EDITORS}

Georgia Benkart

Dave Benson

Richard E. Borcherds

John H. Coates

J-L. Colliot-Thélène

Brian D. Conrad

Hélène Esnault

Hubert Flenner

Edward Frenkel

Andrew Granville

Joseph Gubeladze

Roger Heath-Brown

Ehud Hrushovski

Craig Huneke

Mikhail Kapranov

Yujiro Kawamata

János Kollár

Yuri Manin

Barry Mazur

Philippe Michel
University of Wisconsin, Madison, USA

University of Aberdeen, Scotland

University of California, Berkeley, USA

University of Cambridge, UK

CNRS, Université Paris-Sud, France

University of Michigan, USA

Freie Universität Berlin, Germany

Ruhr-Universität, Germany

University of California, Berkeley, USA

Université de Montréal, Canada

San Francisco State University, USA

Oxford University, UK

Hebrew University, Israel

University of Virginia, USA

Yale University, USA

University of Tokyo, Japan

Princeton University, USA

Northwestern University, USA

Harvard University, USA

École Polytechnique Fédérale de Lausanne
Susan Montgomery

Shigefumi Mori

Raman Parimala

Jonathan Pila

Victor Reiner

Karl Rubin

Peter Sarnak

Joseph H. Silverman

Michael Singer

Vasudevan Srinivas

J. Toby Stafford

Bernd Sturmfels

Richard Taylor

Ravi Vakil

Michel van den Bergh

Marie-France Vignéras

Kei-Ichi Watanabe

Efim Zelmanov

Shou-Wu Zhang
University of Southern California, USA

RIMS, Kyoto University, Japan

Emory University, USA

University of Oxford, UK

University of Minnesota, USA

University of California, Irvine, USA

Princeton University, USA

Brown University, USA

North Carolina State University, USA

Tata Inst. of Fund. Research, India

University of Michigan, USA

University of California, Berkeley, USA

Harvard University, USA

Stanford University, USA

Hasselt University, Belgium

Université Paris VII, France

Nihon University, Japan

University of California, San Diego, USA

Princeton University, USA

PRODUCTION

production@msp.org

Silvio Levy, Scientific Editor

See inside back cover or msp.org/ant for submission instructions.

The subscription price for 2013 is US $\$ 200 /$ year for the electronic version, and $\$ 350 /$ year $(+\$ 40$, if shipping outside the US) for print and electronic. Subscriptions, requests for back issues and changes of subscribers address should be sent to MSP.

Algebra \& Number Theory (ISSN 1944-7833 electronic, 1937-0652 printed) at Mathematical Sciences Publishers, 798 Evans Hall \#3840, c/o University of California, Berkeley, CA 94720-3840 is published continuously online. Periodical rate postage paid at Berkeley, CA 94704, and additional mailing offices.

ANT peer review and production are managed by EditFLOW ${ }^{\circledR}$ from Mathematical Sciences Publishers.

\section{PUBLISHED BY}

- mathematical sciences publishers

nonprofit scientific publishing

http://msp.org/

(C) 2013 Mathematical Sciences Publishers 


\section{Algebra \& Number Theory}

Volume $7 \quad$ No. $8 \quad 2013$

The geometry and combinatorics of cographic toric face rings

1781

Sebastian Cas alaina-Martin, Jesse Leo Kass and Filippo Viviani

Essential $p$-dimension of algebraic groups whose connected component is a torus

Roland LÖTscher, MARK MacDonald, AUREl MEYer and Zinovy

REICHSTEIN

Differential characterization of Wilson primes for $\mathbb{F}_{q}[t]$

DINESH S. THAKUR

Principal $W$-algebras for $\mathrm{GL}(m \mid n)$

JONATHAN BROWN, JONATHAN BRUNDAN and SimON M. GOODWIN

Kernels for products of $L$-functions

NiKOLAOS Diamantis and CORMAC O'SUllivaN

Division algebras and quadratic forms over fraction fields of two-dimensional henselian domains

YONG HU

The operad structure of admissible $G$-covers

DAN PETERSEN

The $p$-adic monodromy theorem in the imperfect residue field case

SHUN OHKUBO

On the Manin-Mumford and Mordell-Lang conjectures in positive characteristic

DAMIAN RÖSSLER 